\title{
Roles of nitric oxide and shear stress in the regulation of microvessel permeability in intact rat mesenteric venules
}

Sulei Xu

Follow this and additional works at: https://researchrepository.wvu.edu/etd

\section{Recommended Citation}

$\mathrm{Xu}$, Sulei, "Roles of nitric oxide and shear stress in the regulation of microvessel permeability in intact rat mesenteric venules" (2014). Graduate Theses, Dissertations, and Problem Reports. 6986.

https://researchrepository.wvu.edu/etd/6986

This Dissertation is protected by copyright and/or related rights. It has been brought to you by the The Research Repository @ WVU with permission from the rights-holder(s). You are free to use this Dissertation in any way that is permitted by the copyright and related rights legislation that applies to your use. For other uses you must obtain permission from the rights-holder(s) directly, unless additional rights are indicated by a Creative Commons license in the record and/ or on the work itself. This Dissertation has been accepted for inclusion in WVU Graduate Theses, Dissertations, and Problem Reports collection by an authorized administrator of The Research Repository @ WVU.

For more information, please contact researchrepository@mail.wvu.edu. 
ROLES OF NITRIC OXIDE AND SHEAR STRESS IN THE REGULATION OF MICROVESSEL PERMEABILITY IN INTACT RAT MESENTERIC VENULES

\author{
Sulei Xu \\ Dissertation submitted to the School of Medicine \\ at West Virginia University \\ In partial fulfillment of the requirements for the degree of \\ Doctor of Philosophy \\ in \\ Cellular and Integrative Physiology Graduate Program
}

Approved by

Pingnian He, MD/Ph.D. Mentor, Committee Chair

Fred L. Minnear, Ph.D.

Robert W. Brock, Ph.D.

Gregory M. Dick, Ph.D.

Stanley D. Yokota, Ph.D.

Geert Schmid-Schonbein, Ph.D

Department of Physiology and Pharmacology

Center for Cardiovascular and Respiratory Sciences

Morgantown, West Virginia

2014

Keywords: endothelial, nitric oxide, intracellular $\mathrm{Ca}^{2+}$, shear stress, endothelial gaps, microvessel permeability

Copyright @ Sulei Xu 


\section{ABSTRACT \\ Roles of nitric oxide and shear stress in the regulation of microvessel permeability}

\section{in intact rat mesenteric venules}

\section{Sulei Xu}

Mechanical forces have been indicated to play important roles in the regulation of inflammatory cell interaction with endothelium resulting in localized leakage formation and contributing to many disease-associated microvascular dysfunctions. However, most of the mechanical force related studies were conducted in vitro. The underlying mechanisms are still controversial. There is a need to investigate how shear stress regulates the endothelial cell (EC) signaling and related vascular barrier function using intact microvessels with experimental conditions closely replicating in vivo situations. The overall aim of my dissertation is to understand the molecular and cellular mechanisms of how shear stress and nitric oxide (NO) regulate microvessel function under physiological and pathological conditions. Studies were conducted on individually perfused intact rat mesenteric venules.

It is well known that shear stress is one of most important regulators in stimulating endothelial cells to produce NO. NO, in addition to being a potent vasodilator, has also been considered a "double edged sword"-mediator in inflammation. Under basal conditions, it prevents leukocyte and platelet adhesion, whereas under inflammatory conditions, the inflammatory mediator-induced excessive NO production contributes to permeability increases. In Chapter 2, we investigated the roles of endothelial basal NO production in leukocyte adhesion and adhesion-induced changes in microvessel permeability. The results indicated that the application of the eNOS specific inhibitor, caveolin-1 scaffolding peptide (CAV), caused reduction of basal NO and promoted ICAM1-mediated leukocyte adhesion through Src activation-mediated ICAM-1 phosphorylation. Also, CAV-induced leukocyte adhesion was uncoupled from leukocyte oxidative burst and microvessel barrier function, unless in the presence of a secondary stimulation.

In Chapter 3, we investigated the roles of shear stress (SS) in the regulation of microvessel permeability and its related EC signaling involving blood cells in individually perfused intact microvessels. Our results demonstrated that in response to a sudden change of SS, transient shear magnitude-dependent increases in $\mathrm{EC}\left[\mathrm{Ca}^{2+}\right]$ i occurred only in vessels perfused with whole blood or perfusate containing RBCs, which was correlated with EC gap formation illustrated by fluorescent microsphere accumulation. Carbenoxolone, a Pannexin 1 inhibitor, significantly reduced shear magnitude-dependent ATP release from RBCs and also abolished SS-induced increases in EC $\left[\mathrm{Ca}^{2+}\right]$ and EC gap formation. Meanwhile, both plasma and whole blood perfusion induced shear magnitude-dependent NO production and eNOS-Ser 1177 phosphorylation.

It is unknown how EC sense SS, but the Glycocalyx (GCX), a layer of proteoglycans covering the endothelium, has been implicated as a mechanical sensor for changes in SS in vitro. The objective of chapter 4 is to identify the changes in GCX in microvessels of 
streptozotocin-induced diabetic rats and evaluate the associated changes in sensing SS and SS-induced NO production in individually perfused venules of diabetic rats. Our results indicated that the impaired GCX in diabetic microvessels enhances EC response to mechanical force and potentiates NO production and EC responses to ATP, resulting in enhanced endothelial gap formation.

Advances in micromanufacturing and microfluidic technologies have enabled a variety of insights into biomedical sciences while curtailing the high experimental costs and complexities associated with animals and in vivo studies. In Chapter 5, we presented and discussed our research work in creating engineered microvessels using a microfluidic platform and demonstrated the formation of the microvascular network in vitro and validated the key features that have been observed in microvessels in vivo. In our future studies, this may provide us a novel platform for studying spatial and temporal change of shear stress in the regulation of microvessel function in a close in vivo situation.

In conclusion, we revealed the role of shear stress and NO in the regulation of endothelial cell signaling and microvessel permeability in vivo, involving blood and nonblood components. The results also suggest the potential in using a microfluidic device in studying the physiological microvessel function. 


\section{ACKNOWLEDGMENTS}

I would like to acknowledge my mentor, Dr. Pingnian He for her guidance and patience as this project evolved. Her faith and dedication to helping me through the many hurdles along the way was invaluable. I could have never accomplished this work without her help. I am also greatly indebted to my other committee members, Dr. Stanley D. Yokota, Dr. Fred Minnear, Dr. Robert W. Brock, Dr. Gregory Dick, and Dr. Matthew A. Boegehold for their

time and effort in attending my seminars and committee meetings, which helped me throughout my project and grant application.

I am very grateful to the people working with me in Dr. He's lab during the past five years: Yan Qian, MD, Dong Yuan, MD, PhD, Gengqian Zhang, MD, PhD, Xueping zhou, $\mathrm{PhD}$, Yanchun Xu, PhD, Christian Stork, PhD, Devendra Sawant, MD, PhD, Qilong Feng, MD, PhD, Yahui Tang, MD, PhD, Mingxia Wang, MS, Anand Kothur, MS, Ge Guo, MS, Petros Evangelos Petrides, MS. I am so fortunate to work with all of you in the lab. I would also like to appreciate our department of physiology and pharmacology faculty members and staff in their time and efforts in organizing seminars and journal clubs.

Last but not least, I would also like to thank my parents, Lizhen Wu and Yaozu Xu, and my grandparents, Ruiying Dong and Jixiang Xu for their unconditional love and support. I would like to dedicate this dissertation to them. Although I am the one went through this $\mathrm{PhD}$ study, in my eyes, they all deserve honorable $\mathrm{PhD}$ degrees in their own life. 


\section{LIST OF ABBREVIATIONS}

AP-CAV

ATP

$\mathrm{AU}$

$\left[\mathrm{Ca}^{2+}\right] \mathrm{i}$

CBX

$\mathrm{cm} \mathrm{H}_{2} \mathrm{O}$

DMSO

DAF-2

DAF-2 DA

DM400

$\Delta \mathrm{FI}_{\mathrm{DAF}}$

EC

$\mathrm{EC}\left[\mathrm{Ca}^{2+}\right] \mathrm{i}$

EGTA

EM

eNOS

FI

FI/A

FMs
Antennapedia caveolin-1 scaffolding domain peptide

Adenosine triphosphate

Arbitrary unit

Intracellular calcium concentration

Cabenoxolone

Centimeter of water

Dimethylsulfoxide

4',5'-diaminofluorescein

4',5'-diaminofluorescein diacetate

Dichroic mirror 400

Net increase in the fluorescence intensity 4',5'-diaminofluorescein

Endothelial cell

Endothelial intracellular calcium concentration

Ethylene glycol-bis(2-aminoethylether)- $N, N, N^{\prime}, N^{\prime}$-tetraacetic acid

Electron microscopy

Endothelial nitric oxide synthase

Fluorescence intensity

Fluorescence intensity per vessel surface area

Fluorescent Microspheres 
g

HEPES

HUVEC

$\mathrm{Jv} / \mathrm{S}$

$\mathrm{KCl}$

$\mathrm{kg}$

L-NMMA

Lp

$\mathrm{MgSO}_{4}$

$\mathrm{MnCl}_{2}$

mo

$\mathrm{mM}$

$\mathrm{NaCl}$

$\mathrm{NaHCO}_{3}$

Na-HEPES

NADPH

NO

PAF

Px1

$P_{d}$
Gram

$\mathrm{N}$-2-hydroxyethylpiperazine-N'-2-ethanesulfonic acid

Human umbilical venous endothelial cell

Volume flux of water per unit area

Potassium chloride

Kilogram

NG-monomethyl-L-arginine

Hydraulic conductivity

Magnesium sulfate

Manganese chloride

Month

Millimolar Micromolar

Sodium chloride

Sodium bicarbonate

Sodium N-2-hydroxyethylpiperazine-N'-2-ethanesulfonic acid

Nicotinamide adenine dinucleotide phosphate

Nitric oxide

Platelet activating factor

Pannexin 1 channel

Solute diffusion permeability 
ROI Region of interest

ROS Reactive oxygen species

SE Standard error

Ser Serine

SOD Superoxide dismutase

SS Shear stress 


\section{TABLE OF CONTENTS}

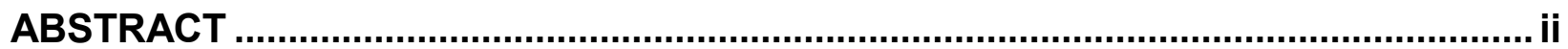

ACKNOWLEDGMENTS ....................................................................................... iv

LIST OF ABBREVIATIONS ....................................................................................

TABLE OF CONTENTS ............................................................................................... viii

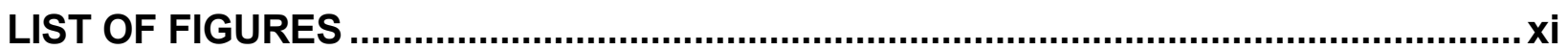

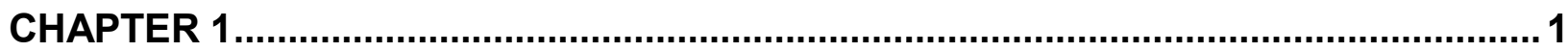

LITERATURE REVIEW ....................................................................................... 1

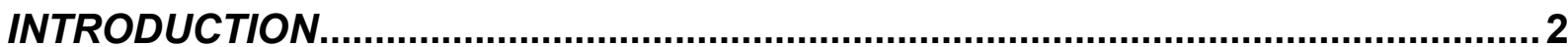

BACKGROUND AND SIGNIFICANCE................................................................ 6

MICROVESSEL PERMEABILITY........................................................................ 6

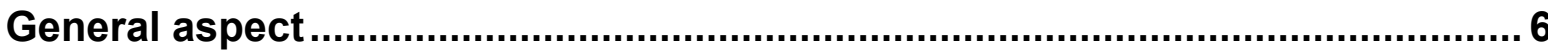

Various experimental preparations in measuring permeability coefficients.... 7 NITRIC OXIDE (NO), MICROVESSEL PERMEABILITY \& LEUKOCYTE ADHESION 8

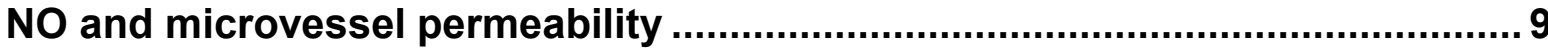

NO and leukocyte adhesion ............................................................................. 10

SHEAR STRESS AND MICROVESSEL PERMEABILITY ..................................... 12

Shear stress and NO production .................................................................. 13

Shear stress and $\mathrm{EC}\left[\mathrm{Ca}^{2+}\right]_{\mathrm{i}}$ changes .................................................... 14

Shear-dependent permeability examined in vitro ........................................ 15

Shear-dependent permeability examined in vivo.......................................... 16

Impaired glycocalyx (shear stress mechanosensor) in disease model........... 18

ENDOTHELIALIZED MICROFLUDIC DEVICE - MINIATURIZED FUNCTIONAL

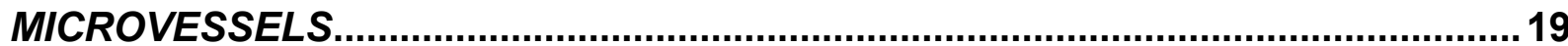

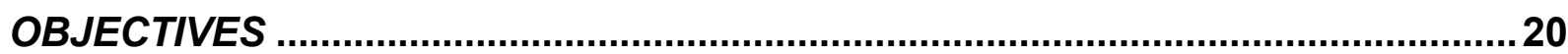

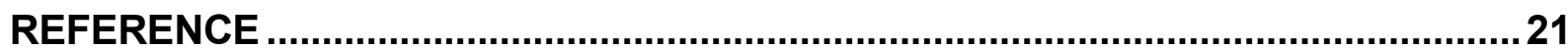

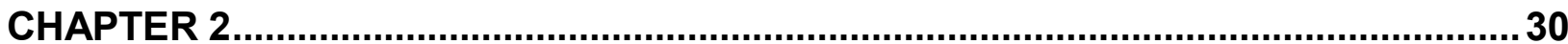

CAVEOLIN-1 SCAFFOLDING DOMAIN PROMOTES LEUKOCYTE ADHESION BY REDUCED BASAL ENDOTHELIAL NITRIC OXIDE-MEDIATED ICAM-1 PHOSPHORYLATION IN RAT MESENTERIC VENULES 30 
ABSTRACT

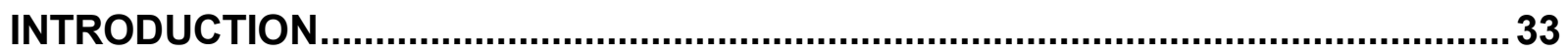

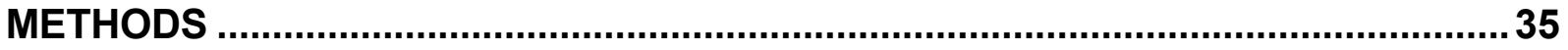

RESULTS

DISCUSSION

REFERENCE

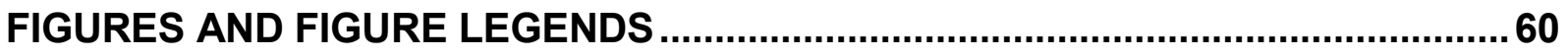

CHAPTER 3

SHEAR STRESS-INDUCED ENDOTHELIAL CELL SIGNALING AND BARRIER FUNCTION IN INTACT MICROVESSELS: INTEGRATIVE ROLES OF FLUIDGENERATED WALL SHEAR STRESS AND SHEAR-INDUCED RELEASES OF ATP

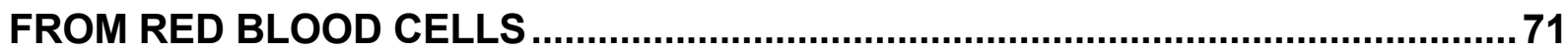

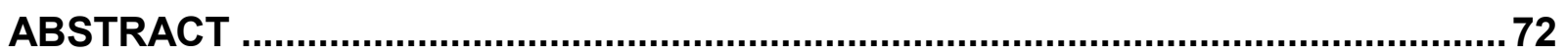

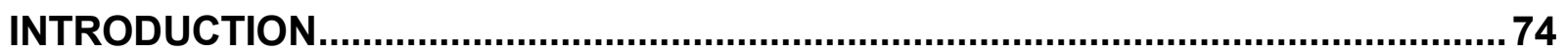

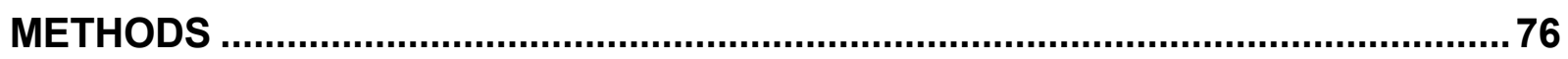

RESULTS

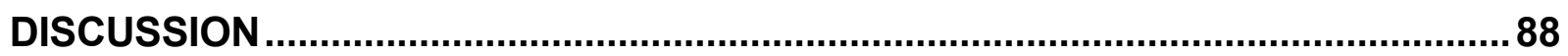

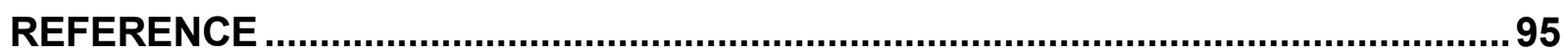

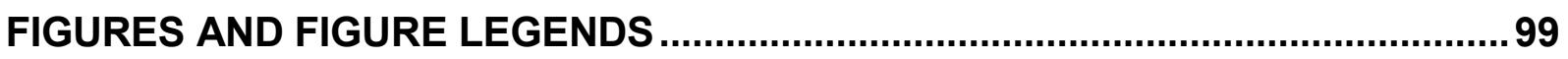

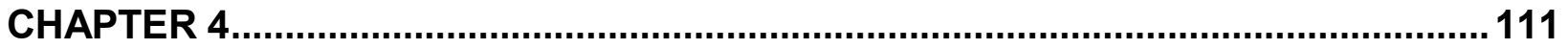

THE LOSS OF GLYCOCALYX IN DIABETIC MICROVESSELS CONTRIBUTES TO THE EXCESSIVE SHEAR STRESS-INDUCED ENDOTHELIAL NITRIC OXIDE

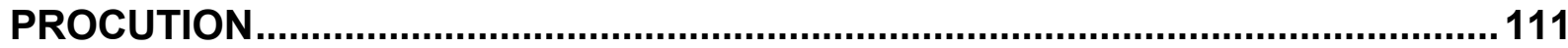

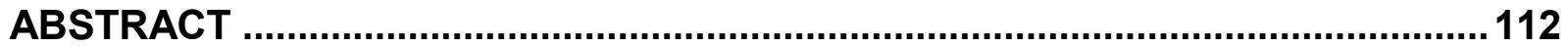

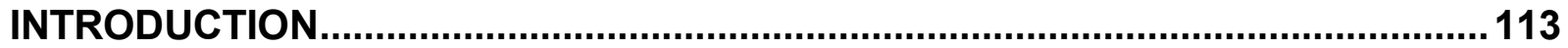

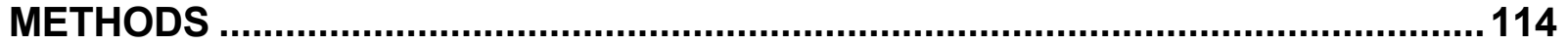

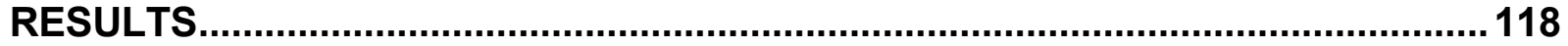

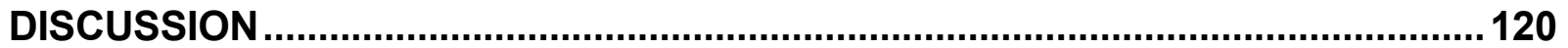

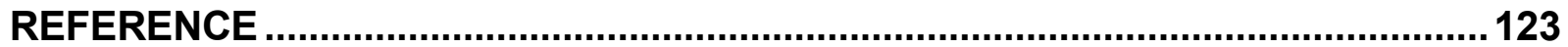

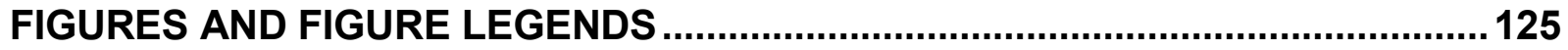

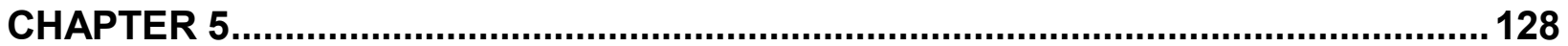

IN VITRO RECAPITULATION OF FUNCTIONAL MICROVESSELS FOR THE STUDY OF ENDOTHELIAL SHEAR RESPONSE, NITRIC OXIDE AND $\left[\mathrm{Ca}^{2+}\right]_{1}$ 


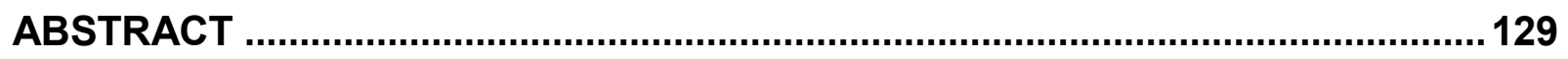

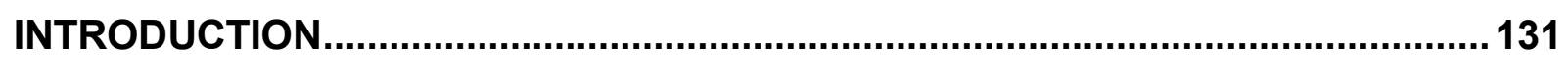

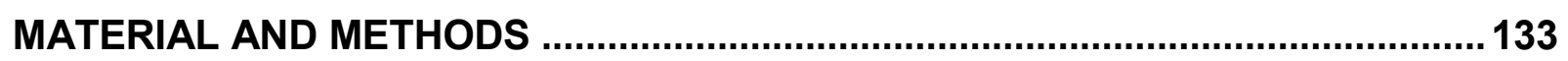

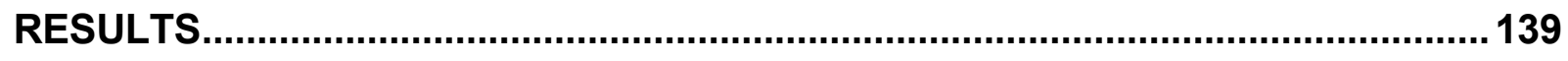

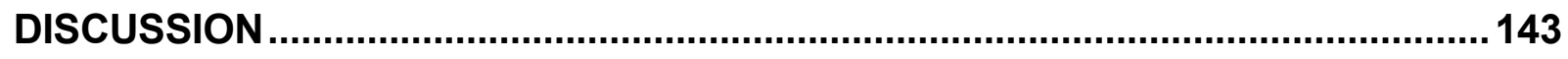

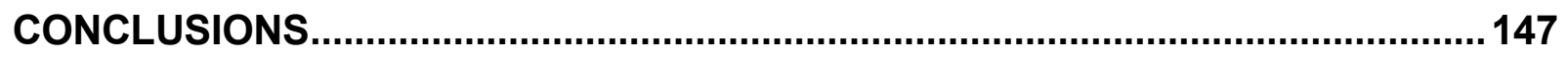

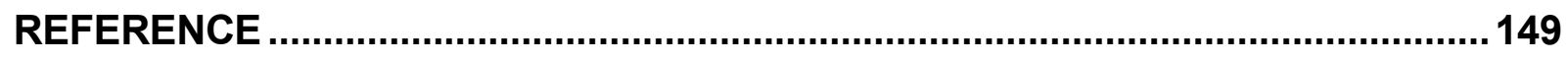

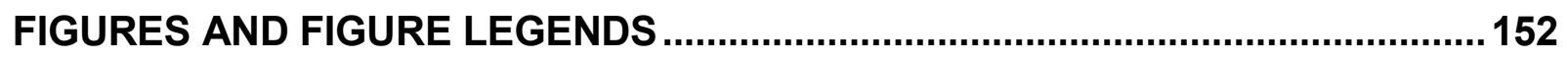

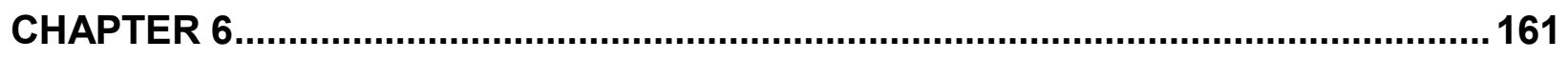

GENERAL DISCUSSION, CONCLUSION AND FUTURE STUDIES .......................161

GENERAL DISCUSSION AND CONCLUSION .................................................. 162

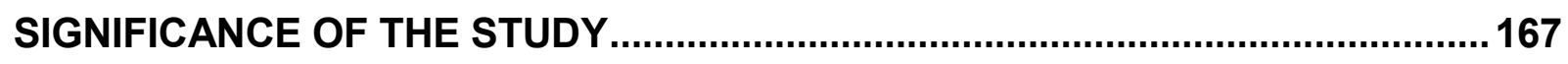

FUTURE STUDIES

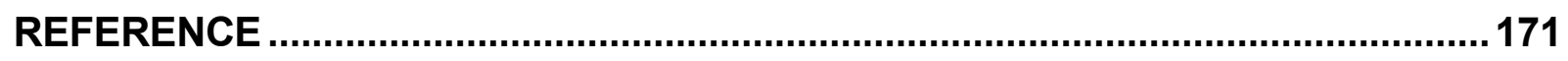

CURRICULUM VITAE 


\section{LIST OF FIGURES}

CHAPTER 2

Page \#

Figure 1. The application of Antennapedia homeodomain (AP)- 60 caveolin-1 (CAV) inhibits basal nitric oxide (NO).

Figure 2 Perfusion of AP-CAV for 30 min has no effect on basal

62 hydraulic conductivity (LP).

Figure 3. Reduction of basal NO by AP-CAV induced significant 63 leukocyte adhesion without increasing $L p$ in the absence of a secondary stimulation.

Figure 4 L-NMMA, a NO synthase (NOS) inhibitor, showed similar 65 effects on leukocyte adhesion and microvessel permeability to those of CAV.

Figure 5 Confocal images of the co-staining of VE-cadherin and 66 adherent leukocytes illustrating that CAV-induced leukocyte adhesion did not change VE-cadherin distribution.

Figure 6 AP-CAV-induced increase in ICAM-1 adhesive capacity causes leukocyte adhesion.

67

Figure 7 AP-CAV-induced leukocyte adhesion involves Src 69 activation-mediated ICAM-1 phosphorylation at tyrosine 526 (Y526).

\section{CHAPTER 3}

Figure 1 Wall Shear stress measurement in intact mesenteric venules.

Figure 2 Blood flow generated-shear stress induced transient increase in $\mathrm{EC}\left[\mathrm{Ca}^{2+}\right]$ in individually perfused venules.

Figure 3 Shear stress-induced EC NO production in rat mesenteric venules

Figure 4 EC eNOS phosphorylation (eNOS-Ser ${ }^{1177}$ and eNOS103 $\mathrm{Thr}^{495}$ ) in response to different magnitudes of shear stress 
generated by either blood or plasma perfusion in the perfused venules.

Figure 5 ATP release from erythrocytes are required for shear stress105 induced transient increase in EC $\left[\mathrm{Ca}^{2+}\right]_{i}$ in individually perfused venules.

Figure $6 \quad$ Blood flow and RBC solution perfusion generated-sudden 107 change of shear stress induced endothelial cell gap formation.

Figure $7 \quad$ Fluid velocity measurement inside cannulation pipette.

\section{CHAPTER 4}

Figure 1 Direct visualization of glycocalyx in rat venules using EM and confocal imaging

Figure 2 Endothelial NO production in response to blood flow126 generated shear stress is significantly enhanced in both diabetic and pronase treated rat mesenteric venules.

Figure 3 Shear stress-induced EC gap formation is enhanced in 127 venules of diabetic rats.

\section{CHAPTER 5}

Figure 1 The schematic fabrication procedures for the microfluidic 152 microchannel network.

Figure 2 The representative confocal images show the HUVECs successfully cultured throughout the inner surfaces of the entire microchannel network.

Figure 3 The representative immunofluorescent confocal images demonstrated the VE-Cadherin junctions formation throughout the entire network.

Figure 4 HUVECs response to the different levels of shear stresses: The morphology change was studied with F-actin staining.

Figure 5 ATP-induced endothelial cell $\left[\mathrm{Ca}^{2+}\right]_{i}$ increase in HUVECs 159 cultured microchannel network. 
Figure 6 ATP-induced NO production increases in HUVECs cultured microchannel network. 


\section{CHAPTER 1}

\section{LITERATURE REVIEW}




\section{INTRODUCTION}

The vascular endothelium lining the interior surface of vascular wall maintains vascular homeostasis by modulating a variety of vascular functions including vascular tone, angiogenesis, vascular remodeling, and inflammatory responses. As a semi-permeable barrier, endothelium is particularly important in regulating the vascular permeability by controlling the exchange of macromolecules and fluid between the blood and interstitial space. Under physiological conditions, the increased vascular permeability enables the extravasation of plasma proteins, immunoglobulins, and others, which serves as one of the important host-defense functions. However, unchecked vascular permeability increase can lead to protein-rich tissue edema, chronic inflammation, and organ dysfunction. Atherosclerosis is one of those pathologies. It has been demonstrated in vivo that atherosclerosis patch is co-localized with sites of elevated endothelial permeability. ${ }^{1,2}$

Endothelial dysfunction is well recognized as the initial step in the atherosclerotic process. ${ }^{3,4}$ A major vasoactive substance released by the endothelium is nitric oxide (NO), originally named as endothelium-derived relaxing factor (EDRF). NO has been recognized to play a pivotal role in maintaining normal vascular functions. Dysregulated NO production

or eNOS (endothelial NO synthase) activity has been proposed as a major mechanism of endothelial dysfunction, contributing to atherosclerosis. However, in addition to being a critical mediator in maintaining vascular homeostasis, NO has also been considered as a "double edged sword"-mediator in inflammation. It has been reported that excessive NO production induced by inflammatory mediators contributes to increased microvessel 
permeability, leading to tissue edema and organ dysfunctions. ${ }^{5-7}$ Basal NO production, on the other hand, has been reported to prevent leukocyte adhesion and platelet aggregation on the vessel wall, therefore, playing an anti-inflammatory role. ${ }^{8-10} \ln$ Chapter 2 of this dissertation, by using caveolin-1 scaffolding domain peptide, an endothelial nitric oxide synthase (eNOS) specific inhibitor, we investigated the role of and underlying mechanisms of endothelial basal NO in preventing leukocyte adhesion and adhesion-induced increases in microvessels permeability.

Wall shear stress (SS) is the tangential force exerted per unit area of blood-endothelium surface. Disturbed shear stress has been reported to promote atherosclerosis and inflammation in the vessel wall. ${ }^{11,12}$ Postcapillary venules are the dominant sites for the occurrence of inflammation and increased microvessel permeability. The role of shear stress in the regulation of venular microvascular barrier function, and how the endothelium senses the change of SS under either physiological or pathological, in particular under diabetic conditions, have not been well explored so far. In Chapters 3 and 4 of this dissertation, studies were conducted to understand the cellular and molecular mechanisms of how SS regulates microvascular barrier function under physiological and diabetic conditions in intact microvessels. In Chapter 3, we investigated the mechanisms of SSinduced changes in microvessel permeability under physiological conditions. We proposed that the glycocalyx on the endothelial surfaces acts as a mechanical sensor of SS to cause alterations in $\mathrm{EC}\left[\mathrm{Ca}^{2+}\right]$, and $\mathrm{NO}$, thus contributing SS-induced changes microvessel permeability. In this study, we measured EC NO, $\left[\mathrm{Ca}^{2+}\right]$ i changes and microvessel 
permeability in response to quantitative alterations of SS in intact rat mesenteric venules. The glycocalyx has been implicated as a mechanical sensor of changes in SS. A variety of diseases such as endotoxemia and hyperglycemia have been reported to cause damage to the glycocalyx coating on the EC surface. ${ }^{13,14}$ However, little is known about the potential effect of degraded glycocalyx on SS-mediated vascular function and how the altered vascular function playing a role in the pathogenesis of these diseases. Therefore, in Chapter 4, studies were conducted to investigate the role of impaired glycocalyx (mechanosensor) in microvessel barrier dysfunction in diabetes by evaluating the ultrastructural changes of EC glycocalyx in the vascular wall and the correlated alterations in SS-induced EC signaling, as well as microvessel permeability using fluorescence and electron microscopic approaches.

To investigate the effects of spatial and temporal variations of SS on endothelial vascular function is a big challenge for us in our study. In vivo microvasculature has unbelievably heterogeneous and complex geometries. It is difficult for us to define and find a certain angle of bifurcated segments in each experiment. Also, due to the time limitation of the in vivo experiments, it is difficult for us to observe the effect of long term SS on endothelial cell signaling and morphological changes, which usually requires more than 48 hours shear exposure. Fortunately, by using up-to-date microfluidic devices lined with primary human umbilical vein endothelial cell, which recapitulated microvessels with fixed geometric pattern, we successfully investigated the long term SS effect on endothelial cell morphology, and how the changes in morphology is associated with changes in cellular 
signaling. In Chapter 5, we investigated changes in vascular endothelial F-actin and VEcadherin to different levels of SS. Also, we quantitatively measured changes in $\mathrm{EC}\left[\mathrm{Ca}^{2+}\right]_{\mathrm{i}}$ and NO production to adenosine triphosphate (ATP).

In summary, the dissertation mainly focuses on investigating the mechanisms of shear stress-mediated changes in microvascular permeability in vivo and in vitro, involving blood and non-blood components 


\section{BACKGROUND AND SIGNIFICANCE \\ MICROVESSEL PERMEABILITY}

\section{General aspect}

Under physiological conditions, the semi-permeable property of vascular wall enables the exchange of gases, ions, and solutes across the vessel wall through endothelial transport. In general, endothelial transport can be divided into paracellular and transcellular pathways. Passive diffusion through the endothelial paracellular route has been found as a dominant exchange pathway for fluid and hydrophilic solutes with molecular radii of up to $3 \mathrm{~nm} .{ }^{15}$ On the other hand, the transcellular vesicular pathway is responsible for the active transport of macromolecules, such as albumin. In order to quantitatively define the microvessel exchange property, microvessel permeability coefficients, which reflect and represent the barrier function of microvascular walls to a specific substance, were adopted in various experimental measurements as shown in Table 1.

Table 1 Membrane permeability coefficients

Permeability coefficient

Hydraulic conductivity

Diffusional permeability

Solvent drag (ultrafiltration)

Osmotic reflection coefficient

Symbol
$L_{p}$
$P_{d}$
$\sigma_{f}$
$\sigma_{d}$

In the studies of this dissertation, the hydraulic conductivity $\left(L_{p}\right)$, which represents the fluid permeability across the vessel wall was evaluated in individually cannulated intact venules. The Lp was quantitatively measured by calculating the volume flux of water across the 
vessel wall under a certain driving force of pressure difference. It is well illustrated in the Starling Equation. ${ }^{16}$

$$
J_{V}=\left(L_{p} S\right)\left[\left(P_{c}-P_{i}\right)-\sigma\left(\pi_{c}-\pi_{i}\right)\right]
$$

$J_{v}$ : Volume flux of fluid; $L_{p}$ : Hydraulic conductivity; $S$ : Surface area; $P_{c}$ :Capillary fluid hydrostatic pressures; $\mathrm{P}_{\mathrm{i}}$ Interstitial fluid hydrostatic pressures; $\pi c$ : Capillary colloid osmotic (oncotic) pressures; $\pi t:$ Interstitial colloid osmotic (oncotic) pressures; $\sigma: O s m o t i c ~ r e f l e c t i o n$ coefficient of vessel wall ( 0 if membrane is fully permeable to transported molecular species and 1 if the membrane is impermeable).

\section{Various experimental preparations in measuring permeability coefficients}

Microvascular permeability coefficients can be measured from perfused whole organs, single cannulated microvessels, to monolayers of cultured endothelial cells. They all have their advantages and disadvantages.

Permeability coefficient measured in whole organs, isolated tissues or whole animals, involves minimal interference with experimental handling, which is closest to the native situation in vivo. However, those studies usually overlook changes in transmural pressure gradients, changes in exchanging surface area, and presence of blood cells in the regulation of vascular permeability. Especially, in those animal disease models, the changes of cell and vascular morphology can greatly affect hemodynamic alternation, which usually results in inconclusive measurements.

The use of in vitro monolayers of cultured ECs allows the investigation of cellular and molecular mechanisms of the regulation of endothelial permeability. However, cultured 
endothelium has been reported to be a pro-inflammatory endothelial phenotype in comparison to the native endothelium in vivo. 17 Also, multidimensional protein identification revealed that $41 \%$ of proteins expressed in vivo were not detected in vitro, indicating that distinct in vivo protein expression is apparently regulated by the tissue microenvironment and cannot yet be duplicated in standard cell culture.$^{18} \mathrm{It}$ raises serious concerns when directly applying the knowledge we gained from in vitro to those in vivo.

Using a single cannulated microvessel in the study of microvessel permeability is the main technique we adopted in this dissertation. The primary advantages of this approach is that it allows the direct and precise control of the conditions of perfusion for a defined segment of microvessel while directly measuring the real time changes of the vessel surface area, the difference in pressure, and concentration of solutes across the vessel walls. The limitations of studies on single vessels are i) experimental preparations are restricted to several convenient vessel types, such as mesenteric vessels and cremaster vessels; ii) there is an experimental duration limit for these preparations and iii) each experiment preparation is also quite time consuming.

\section{NITRIC OXIDE (NO), MICROVESSEL PERMEABILITY \& LEUKOCYTE ADHESION}

NO has been recognized as an important cardiovascular signaling factor that is not only a potent vasodilator regulating vascular tone and blood pressure, but also plays an important role in the regulation of microvessel permeability and leukocyte adhesion. ${ }^{8,19-}$ ${ }^{22} \mathrm{NO}$ is biosynthesized from L-arginine through the enzyme NO synthase (NOS). There are three main isoforms of the enzyme called neuronal NOS (nNOS), inducible NOS 
(iNOS), and endothelial NOS (eNOS). Among those, eNOS has been found to be the dominant constitutive enzyme responsible for NO production in the cardiovascular system.

\section{NO and microvessel permeability}

For decades, a growing body of literature suggested that NO modulates the movement of fluid and protein from the vasculature. However, the results obtained from whole organ, single vessel, and endothelial monolayer studies are often not consistent. Numerous experimental evidence suggest that endogenous NO increases microvascular permeability while an equal amount of evidence indicates that NO inhibits increases in microvascular permeability. Since the early 1990s, Kubes et al. and others showed that nonselective NO synthase inhibition increased vascular leakage in the presence of blood, suggesting a protective role of NO in barrier function. ${ }^{8,23-25}$ Almost at the same time, others showed that inhibition of NO prevented permeability increases in response to inflammatory mediators and other physical stimulates. ${ }^{7,22,26-35}$ Those conflicting results were derived from different experimental approaches, organs, tissue conditions, and animal species. Our previous findings showed that excessive NO production induced by inflammatory stimuli is responsible for the increases in microvessel permeability ${ }^{26-29}$. Our present study in this dissertation was to investigate the role of basal NO production in the regulation of microvessel permeability using the same experimental preparation. Combined, these two results revealed the dual actions of $\mathrm{NO}$ on endothelial functions at basal and inflammatory conditions, which provides a unified perspective of the roles of NO in the regulation of endothelial function and microvessel permeability. 
Caveolin-1, the primary coating protein of caveolae, has been recognized as a key regulator of eNOS activation through binding of its "scaffolding domain". In Chapter 2, by using a chimeric peptide with a cellular internalization sequence (homeodomain of antennapedia (AP) fused to the caveolin-1 scaffolding domain (amino acids 82-101), which we named as AP-CAV, we were able to specifically inhibit eNOS and its basal NO production. Our results suggested that the reduced basal NO did not directly increase microvessel permeability. It is consistent with results from eNOS knockout mice that basal permeability is not affected by the absence of eNOS. ${ }^{36}$

\section{NO and leukocyte adhesion}

Before we discuss the role of NO in leukocyte adhesion, it is important to briefly review the process of the recruitment of leukocytes into inflamed tissues. The adhesion of leukocytes to vascular endothelium is one of the most dramatic cellular responses of the inflammatory process. The recruitment process has been intensively investigated and characterized into several main steps. This recruitment process requires specific adhesion glycoproteins in the interaction of leukocytes with ECs. Initially, leukocytes marginate to the vascular wall due to both physical and chemical factors, such as piled up red blood cells behind leukocytes and the secretion of chemokines. ${ }^{37,} 38$ Then the leukocytes start to roll and tether in a process involving a group of selectin family proteins, such as L-selectin at the surface of leukocytes, and E-selectin and P-selectin expressed on activated ECs. ${ }^{39}$ After cytokines activate both leukocytes and endothelium, leukocytes get into a stationary state in which the leukocyte remains firmly attached to the endothelial cell surface, without rotational motion. This is mediated by the CD11/CD18 on the leukocytes and ICAM-1 or 
VCAM-1 on the ECs. ${ }^{19}$ Following the firm adhesion, leukocytes may transmigrate from postcapillary venules into the interstitial space through a paracellular or transcellular route, a process involves PECAM-1 and ICAM-1. ${ }^{38}$

Studies performed in various in vitro or animal models have shown the role of NO in anti-leukocyte adhesion. This was supported by the facts that non-specific NOS inhibitors, such as NG-Monomethyl-L-arginine (L-NMMA) or NG-nitro-L-arginine methyl ester (LNAME), evoked marked increases in the attachment of leukocytes to endothelial cells. ${ }^{9,40-}$ 42. Also, it has been shown that NO donors (nitroprusside, SIN-I) and superoxide dismutase attenuate or prevent the leukocyte adherence induced by different inflammatory stimuli. ${ }^{43}$ Although it is well known that leukocyte-endothelial interaction is dependent on the expression or activity of adhesion molecules, the role of NO in regulating those molecules has not been fully elucidated. In vivo studies on cats and rats suggested that giving LNMMA or L-NAME systemically causes the increase of CD11/CD18 complex expression on leukocytes ${ }^{9,41,44}$. It was also shown that superfusion of the rat mesentery with L-NAME significantly increased leukocyte rolling and adherence in mesenteric venules through enhanced the expression of P-selectin. ${ }^{45}$ In addition, in vitro studies indicated that the antiadhesive role of NO could also be attributed by changing the expression or activity of ICAM1 and VCAM-1. ${ }^{46,47}$ However, those observations are not consistent with each other due to different animal species, tissue origins and experimental preparations.

In Chapter 2, we found that perfusion of AP-CAV in rat postcapillary venules, not only inhibited the basal NO production, it also increased adhesive binding sites of ICAM-1 for leukocyte adhesion. Moreover, we showed significant increases in phospho-(Y526)-ICAM- 
1 staining following AP-CAV perfusion, which was abolished by a Src kinase inhibitor, PP1. Consistent with our findings, overexpression of caveolin-1 was shown to reduce NO production in vitro ${ }^{48-50}$, and eNOS-deficient mice showed an increased number of adherent leukocytes. ${ }^{51}$ These results demonstrated that the reduced basal NO does not directly increase permeability, but rather primes the microvascular endothelial cells into an adhesive state.

\section{SHEAR STRESS AND MICROVESSEL PERMEABILITY}

Blood flow-generated hemodynamic forces are the most physiologically relevant factors for the maintenance and regulation of the homeostasis of endothelial cells. Hemodynamic forces can be divided into two major vectors which are tensile stress (pressure) and SS. The stress perpendicular to the vessel wall is defined as tensile stress, which imposes circumferential stretch to the tissue. The stress parallel to the vessel wall is defined as SS, which serves as frictional force acting on the surface of endothelial cells. There has been substantial evidence that early atherosclerotic plaques develop mainly in regions of low wall SS, such as arterial bifurcations, whereas vascular regions with high laminar wall SS are less prone to develop atherosclerotic plaques. ${ }^{11,52}$ Disrupted endothelial function, including

vascular barrier dysfunction, have been considered as the major causes of atherosclerosis. ${ }^{3,53}$ Thus, it is essential to study how SS regulates the endothelial cell signaling and related vascular barrier function. 


\section{Shear stress and NO production}

Although there is a large body of studies trying to elucidate how SS induce EC to generate NO, the knowledge of SS-induced NO production still remains unclear. Firstly, due to low concentration and short half-life of $\mathrm{NO}$, it is difficult to accurately measure it in real time. In addition, it is particularly challenging in detecting NO produced in response to shear stress due to changes in flow caused other changes such as chemical degradation. Several direct and indirect techniques have been established to measure NO. Indirect methods involve measurement of nitrite or nitrate (the end product of NO metabolism) using the Griess reagent, ${ }^{54}$ detecting soluble guanylyl cyclase (sGC) (which is activated after NO binding) using chemiluminescence methods, ${ }^{55}$ and measurement of cGMP, which is produced by activated SGC, using a sGC derived fluorescence indicator. ${ }^{56}$ Direct measurement of NO includes electrodes and 4',5'-diaminofluorescein diacetate (DAF-2 DA), a cell permeable fluorescent probe. Up to now, electrodes remain the dominant method for direct NO measurement. However, since NO has a sharply decreased gradient in concentration away from the source of production, the precise and reproducible positioning of the electrode becomes a critical limiting factor in this method. In Chapter 3 , we used DAF-2 as a NO indicator to directly measure the NO production induced by flow shear stress. The drawback of this dye is its relative short maintaining time (about 1 to 1.5 hrs) inside the cells in vivo. Secondly, prior in vitro studies on the role of $\mathrm{Ca}^{2+}$ in shear stress-mediated NO production have yielded conflicting results. Some studies suggested that the increase in NO production was independent of extracellular $\mathrm{Ca}^{2+}$, suggesting the

release of $\mathrm{Ca}^{2+}$ from intracellular stores as an important source responsible for the 13 
increased EC $\left[\mathrm{Ca}^{2+}\right] .{ }^{57,58,59}$ However, other studies suggested that removal of extracellular $\mathrm{Ca}^{2+}$ blocked flow-induced NO release from intact vessels and from cultured endothelial cells. ${ }^{60}$ Also, for those cells grown on collagen-coated microcarrier beads, either removal of medium $\mathrm{Ca}^{2+}$ by using EGTA or chelation of intracellular $\mathrm{Ca}^{2+}$ by adding BAPTA did not block flow-mediated NO release. ${ }^{61}$ Those varied results may be due to the difference in cultured cell origins, variable concentrations of ATP in culture media, and different conditions of flow (laminar or turbulence flow). Our results demonstrated that the changes of wall SS generated by perfusion of microvessels with either blood or plasma, induced NO production in vivo. Additionally, only blood perfusion induced transient increase in EC $\left[\mathrm{Ca}^{2+}\right] \mathrm{i}$, suggesting that wall shear stress induced NO production may be calcium independent.

\section{Shear stress and $E C\left[\mathrm{Ca}^{2+}\right]_{i}$ changes}

$\mathrm{Ca}^{2+}$ is a major internal cell signaling component. It mediates a large range of cell functions such as cytoskeleton reorganization, apoptosis and contraction. A number of studies from different research groups have demonstrated a link between pro-inflammatory mediator-induced $\mathrm{Ca}^{2+}$ signaling and endothelial permeability ${ }^{62-65}$ It has been shown that inhibition of $\mathrm{Ca}^{2+}$ influx and depletion of the extracellular $\mathrm{Ca}^{2+}$ abolished or significantly attenuated the inflammatory stimuli-induced increase in microvessel permeability. ${ }^{62,} 65$ These results indicate that the increase in $\mathrm{EC}\left[\mathrm{Ca}^{2+}\right]_{i}$ is an essential signal for inflammatory mediator-induced increases in microvessel permeability. It is proposed that blood flowgenerated SS modulates endothelial calcium response via two pathways under physiological condition. ${ }^{66}$ Firstly, SS enhances the transfer of blood-borne agonists, e.g. 
those inflammatory stimuli, to the endothelial cell surface, which mediates the increase of EC $\left[\mathrm{Ca}^{2+}\right]_{i}$. Secondly, the permeability of the cell membrane to extracellular calcium increases upon pure exposure to SS. The increase of $\mathrm{EC}\left[\mathrm{Ca}^{2+}\right]$ induced by the change in SS was thought to be mainly due to the influx of extracellular $\mathrm{Ca}^{2+}$ across the cell membrane, which is elicited by the disappearance of $\mathrm{Ca}^{2+}$ response to SS after the removal of extracellular $\mathrm{Ca}^{2+} .{ }^{67}$ However, it has been controversial for decades whether exposure of EC to flow itself is accompanied by elevated cytoplasmic calcium concentration. ${ }^{57,68,69}$ In those studies, the acute change of SS induced varied magnitude of increase in $\left[\mathrm{Ca}^{2+}\right]$, and the effects were not related to the magnitude of the SS. ${ }^{68,69}$ One of the main differences for the inconsistent observations in those studies is the amount of ATP in culture cell medium. ${ }^{69,70}$ It has been shown that in the absence or a low concentration of ATP, the change of EC $\left[\mathrm{Ca}^{2+}\right]_{\mathrm{i}}$ in response to the change of SS is not significant or weak. ${ }^{67,69,71}$ In Chapter 3, we showed that only blood flow-generated sudden changes in SS, but not plasma or similar viscous dextran-albumin-ringer fluid perfusion, induced a transient EC $\left[\mathrm{Ca}^{2+}\right] \mathrm{i}$ increase in a shear-magnitude-dependent manner. More interestingly, the shearinduced ATP release from red blood cells contributes to the transient mobilization of EC $\left[\mathrm{Ca}^{2+}\right]$.

\section{Shear-dependent permeability examined in vitro}

The development of different types of flow devices and successful lining of cultured endothelial monolayers on the test surfaces enables the investigation of the relationships between SS and the endothelial function in vitro. Up to now, there are four types of flow devices used to define SS: 1) parallel-disk viscometer; 2) the cone and plate viscometer; 
3) parallel plate flow chamber; and 4) microfluidic device. Easy access to the cells and ready manipulation of the flow characteristics provided a great amount of information in understanding how cell response to the SS from changes in gene expression, cell signaling, to cell morphology and function. It has been shown on Bovine Aortic Endothelial Cells (BAEC) and Human Umbilical Vein Endothelial Cells (HUVEC) monolayers that flow SS can increase Lp and electrical impedance mediated by NO. ${ }^{72-75}$ Meanwhile, those two types of cells have different magnitude and duration of permeability increase in response

to SS. The diverse responses to SS in different cell types may due to their difference in intracellular signaling pathways and transport properties ${ }^{76}$ Although in vitro studies provide us valuable insights into potential mechanisms involved in the regulation of SS-mediated changes in endothelial permeability, whether it can reflect the truth in vivo is still a question. In fact, there are several significant differences between the cultured cell and in vivo situation. Firstly, most of the in vitro studies were conducted with cells grown under static conditions, and then exposed to SS during measurements. Thus, the observed response has been doubted as the adaptation to a changed SS environment rather than a direct effect of fluid SS on endothelium functions. Secondly, cultured cells have a proinflammatory phenotype. Its basal permeability is $\sim 10$ fold higher than that in intact venules. ${ }^{77}$ Thirdly, most in vitro studies did not include blood cells components in generating SS, which differs from the in vivo situation where SS is generated by blood flow.

\section{Shear-dependent permeability examined in vivo}

Post-capillary venules are the main sites where the permeability increase and recruitment of leukocytes occur during inflammation. Acute responses to changes in shear 
stress have its physiological significance in monitoring changes in activity level or the demands to a specific organ on a minute-to-minute basis. ${ }^{78}$ Kajimura and Michel showed a positive correlation between flow velocity and potassium ion permeability in rats venules. ${ }^{79}$ Also, Montermini et al. demonstrated sodium fluorescein permeability increases linearly to flow velocity in both rat and frog venules. ${ }^{78}$ However, although there were a few in vivo studies which investigated whether the shear stress (by increasing either perfusion pressure or shear rate) affect microvessel $L p$ in frog or rat mesentery venules, the clear pictures regarding how shear regulates the microvessel barrier function still remain controversial. ${ }^{80-82}$ By using a double cannulation method, Neal and Bates could not find a positive correlation between increased flow rate and $L p$ in microvessels of frog mesentery..$^{80}$ Using modified Landis technique, Adamson et al. did not observe a correlation between shear stress and Lp in vessels perfused in the orthograde direction. ${ }^{82}$ Conversely, using the same technique, Williams showed a dramatic increase in Lp in response to a step change in shear stress in rat venules. ${ }^{83}$ In addition, Kim et al. observed that changes in Lp were positively correlated with the magnitude of acute changes in shear stress in autoperfused microvessels. ${ }^{84}$ The controversy of those studies may due to the differences in methods, (e.g. single or double cannulation, and autoperfusion), as well as the difference in the nature of the perfusate (e.g. in the presence or absence of blood cells). In this study, we investigated the precise role of blood or non-blood flow-generated acute increase of wall shear stress on endothelial cell signaling and microvessel permeability. 


\section{Impaired glycocalyx (shear stress mechanosensor) in disease model}

Although a vast number of studies have been undertaken to investigate the mechanisms of shear stress-mediated mechanotransduction, the mechanosensor and sensing signaling, which recognizes shear stress and passes the mechanical force into intracellular signaling pathway have not been identified. So far, there are several mechanosensor candidates have been proposed on the surface of EC, such as ion channels,${ }^{85}$ G-protein-coupled receptors (GPCRs), ${ }^{86}$ caveolae, ${ }^{87}$ adhesion proteins, ${ }^{88}$ and the glycocalyx. ${ }^{89}$

The glycocalyx is an organized negatively charged mesh of glycoproteins, proteoglycans, glycosaminoglycans (GAGs), and associated plasma proteins of proteoglycans covering the endothelium. Involvement of the glycocalyx in EC responses to shear stress has been demonstrated by enzymatic removal Heparan Sulfate using heparinase III on BAEC completely inhibited NO production induced by fluid shear stress. 90 However, the glycocalyx in cultured cells is considerably thinner than that in intact venules. ${ }^{91}$ Therefore, it is important to investigate the roles of the glycocalyx in shear stressmediated endothelium functions in intact microvessels.

Besides its function as a mechanosensor, glycocalyx serves throughout the vasculature as an aegis, protecting blood vessels from coagulation, inflammation and atherosclerosis. Decreased glycocalyx volume has been reported in diabetic patients. ${ }^{14} \mathrm{~A}$ study in frog microvessels showed an increased microvessel hydraulic conductivity (Lp) upon partial digestion of the endothelial glycocalyx..$^{92}$ Our previous study found an increased basal permeability and enhanced permeability responses to inflammatory mediators in venules 
of streptozotocin-induced diabetic diabetic rats. ${ }^{93}$ Whether the increased permeability in diabetic rats is a result of an impaired glycocalyx remains unknown. In Chapter 4, we conducted experiments to test the hypothesis that hyperglycemia-induced damages to the glycocalyx contribute to the increases in microvessel permeability in diabetic rats. Also, we investigated the effect of impaired glycocalyx on changes in shear stress-induced nitric oxide (NO) production in individually perfused venules of diabetic rats.

\section{ENDOTHELIALIZED MICROFLUDIC DEVICE - MINIATURIZED FUNCTIONAL MICROVESSELS}

The idea of using microfluidic devices as miniaturized whole organ or tissue is much the same way as a microelectronic circuit is an entire computer on a chip. Since the idea came out, microfluidic technology has given great promise to overcome the challenge of performing in vitro experiments with more physiologically realistic setups but still easier and cheaper to handling than animal studies. In Chapter 5 , in vitro growth microvessel network using the microfluidic approach and characterized the ECs' junction, responses to shear stresses, NO production, and endothelial $\left[\mathrm{Ca}^{2+}\right]_{i}$. The results demonstrated that the model successfully recapitulates key aspects and functions of the in vivo microvessels. 


\section{OBJECTIVES}

Specific Aim 1 to investigate the roles of exogenously applied CAV in endothelial basal NO production, leukocyte adhesion, and adhesion-induced changes in microvessel permeability.

Specific Aim 2 is to investigate the cellular and molecular mechanisms for how shear stress regulates microvessel functions under physiological conditions.

Specific Aim 3 is to investigate the role of impaired glycocalyx in microvessel barrier dysfunction in diabetes.

Specific Aim 4 is to demonstrate in vitro formation of microvessels that recapitulate key features of the microvessels in vivo. 


\section{REFERENCE}

1. Miao H, Hu YL, Shiu YT, Yuan S, Zhao Y, Kaunas R, Wang Y, Jin G, Usami S, Chien S. Effects of flow patterns on the localization and expression of VE-cadherin at vascular endothelial cell junctions: in vivo and in vitro investigations. $J$ Vasc Res. 2005;42(1):77-89.

2. Nielsen LB, Nordestgaard BG, Stender S, Kjeldsen K. Aortic permeability to LDL as a predictor of aortic cholesterol accumulation in cholesterol-fed rabbits. Arterioscler Thromb. 1992;12(12):1402-1409.

3. Davignon J, Ganz P. Role of endothelial dysfunction in atherosclerosis. Circulation. 2004;109(23 Suppl 1):III27-32.

4. Sitia S, Tomasoni L, Atzeni F, Ambrosio G, Cordiano C, Catapano A, Tramontana S, Perticone F, Naccarato P, Camici P, Picano E, Cortigiani L, Bevilacqua M, Milazzo L, Cusi D, Barlassina C, Sarzi-Puttini P, Turiel M. From endothelial dysfunction to atherosclerosis. Autoimmun Rev.9(12):830-834.

5. He P, Liu B, Curry FE. Effect of nitric oxide synthase inhibitors on endothelial [Ca2+]i and microvessel permeability. Am J Physiol. 1997;272(1 Pt 2):H176-185.

6. Wu HM, Huang $Q$, Yuan $Y$, Granger HJ. VEGF induces NO-dependent hyperpermeability in coronary venules. Am J Physiol. 1996;271(6 Pt 2):H2735-2739.

7. Mayhan WG. Nitric oxide accounts for histamine-induced increases in macromolecular extravasation. Am J Physiol. 1994;266(6 Pt 2):H2369-2373.

8. Kubes $\mathrm{P}$, Granger DN. Nitric oxide modulates microvascular permeability. Am J Physiol. 1992;262(2 Pt 2):H611-615.

9. Kubes $P$, Suzuki M, Granger DN. Nitric oxide: an endogenous modulator of leukocyte adhesion. Proc Natl Acad Sci U S A. 1991;88(11):4651-4655.

10. Radomski MW, Palmer RM, Moncada S. Endogenous nitric oxide inhibits human platelet adhesion to vascular endothelium. Lancet. 1987;2(8567):1057-1058.

11. Malek AM, Alper SL, Izumo S. Hemodynamic shear stress and its role in atherosclerosis. JAMA. 1999;282(21):2035-2042.

12. Strony J, Beaudoin A, Brands D, Adelman B. Analysis of shear stress and hemodynamic factors in a model of coronary artery stenosis and thrombosis. Am J Physiol. 1993;265(5 Pt 2):H1787-1796.

13. Marechal X, Favory R, Joulin O, Montaigne D, Hassoun S, Decoster B, Zerimech F, Neviere R. Endothelial glycocalyx damage during endotoxemia coincides with 
microcirculatory dysfunction and vascular oxidative stress. Shock. 2008;29(5):572576.

14. Nieuwdorp M, van Haeften TW, Gouverneur MC, Mooij HL, van Lieshout MH, Levi M, Meijers JC, Holleman F, Hoekstra JB, Vink H, Kastelein JJ, Stroes ES. Loss of endothelial glycocalyx during acute hyperglycemia coincides with endothelial dysfunction and coagulation activation in vivo. Diabetes. 2006;55(2):480-486.

15. Pappenheimer JR, Renkin EM, Borrero LM. Filtration, diffusion and molecular sieving through peripheral capillary membranes; a contribution to the pore theory of capillary permeability. Am J Physiol. 1951;167(1):13-46.

16. Starling $\mathrm{EH}$. On the Absorption of Fluids from the Connective Tissue Spaces. $J$ Physiol. 1896;19(4):312-326.

17. Curry FR, Adamson RH. Vascular permeability modulation at the cell, microvessel, or whole organ level: towards closing gaps in our knowledge. Cardiovasc Res.87(2):218-229.

18. Durr E, Yu J, Krasinska KM, Carver LA, Yates JR, Testa JE, Oh P, Schnitzer JE. Direct proteomic mapping of the lung microvascular endothelial cell surface in vivo and in cell culture. Nat Biotechnol. 2004;22(8):985-992.

19. Kvietys PR, Granger DN. Role of reactive oxygen and nitrogen species in the vascular responses to inflammation. Free Radic Biol Med. 2012;52(3):556-592.

20. Cirino G, Fiorucci S, Sessa WC. Endothelial nitric oxide synthase: the Cinderella of inflammation? Trends Pharmacol Sci. 2003;24(2):91-95.

21. Kuhlencordt PJ, Rosel E, Gerszten RE, Morales-Ruiz M, Dombkowski D, Atkinson WJ, Han F, Preffer F, Rosenzweig A, Sessa WC, Gimbrone MA, Jr., Ertl G, Huang PL. Role of endothelial nitric oxide synthase in endothelial activation: insights from eNOS knockout endothelial cells. Am J Physiol Cell Physiol. 2004;286(5):C11951202.

22. Duran WN, Breslin JW, Sanchez FA. The NO cascade, eNOS location, and microvascular permeability. Cardiovasc Res.87(2):254-261.

23. Kurose I, Wolf R, Grisham MB, Granger DN. Effects of an endogenous inhibitor of nitric oxide synthesis on postcapillary venules. Am J Physiol. 1995;268(6 Pt 2):H2224-2231.

24. Kurose I, Kubes P, Wolf R, Anderson DC, Paulson J, Miyasaka M, Granger DN. Inhibition of nitric oxide production. Mechanisms of vascular albumin leakage. Circ Res. 1993;73(1):164-171. 
25. Rumbaut RE, Wang J, Huxley VH. Differential effects of L-NAME on rat venular hydraulic conductivity. Am J Physiol Heart Circ Physiol. 2000;279(4):H2017-2023.

26. Zhu L, Schwegler-Berry D, Castranova V, He P. Internalization of caveolin-1 scaffolding domain facilitated by Antennapedia homeodomain attenuates PAFinduced increase in microvessel permeability. Am J Physiol Heart Circ Physiol. 2004;286(1):H195-201.

27. Zhu L, He P. Platelet-activating factor increases endothelial [Ca2+]i and NO production in individually perfused intact microvessels. Am J Physiol Heart Circ Physiol. 2005;288(6):H2869-2877.

28. Zhou X, He P. Temporal and spatial correlation of platelet-activating factor-induced increases in endothelial $[\mathrm{Ca}(2)(+)] \mathrm{i}$, nitric oxide, and gap formation in intact venules. Am J Physiol Heart Circ Physiol. 2011;301(5):H1788-1797.

29. Zhou X, He P. Endothelial [Ca2+]i and caveolin-1 antagonistically regulate eNOS activity and microvessel permeability in rat venules. Cardiovasc Res. 2010;87(2):340-347.

30. Bernatchez PN, Bauer PM, Yu J, Prendergast JS, He P, Sessa WC. Dissecting the molecular control of endothelial NO synthase by caveolin-1 using cell-permeable peptides. Proc Natl Acad Sci U S A. 2005;102(3):761-766.

31. Bucci M, Gratton JP, Rudic RD, Acevedo L, Roviezzo F, Cirino G, Sessa WC. In vivo delivery of the caveolin-1 scaffolding domain inhibits nitric oxide synthesis and reduces inflammation. Nat Med. 2000;6(12):1362-1367.

32. Bucci M, Roviezzo F, Posadas I, Yu J, Parente L, Sessa WC, Ignarro LJ, Cirino G. Endothelial nitric oxide synthase activation is critical for vascular leakage during acute inflammation in vivo. Proc Natl Acad Sci U S A. 2005;102(3):904-908.

33. Mayhan WG. Role of nitric oxide in modulating permeability of hamster cheek pouch in response to adenosine 5'-diphosphate and bradykinin. Inflammation. 1992;16(4):295-305.

34. Hughes SR, Williams TJ, Brain SD. Evidence that endogenous nitric oxide modulates oedema formation induced by substance P. Eur J Pharmacol. 1990;191(3):481-484.

35. Yuan Y, Granger HJ, Zawieja DC, Chilian WM. Flow modulates coronary venular permeability by a nitric oxide-related mechanism. Am J Physiol. 1992;263(2 Pt 2):H641-646.

36. Fukumura D, Gohongi T, Kadambi A, Izumi Y, Ang J, Yun CO, Buerk DG, Huang $\mathrm{PL}$, Jain RK. Predominant role of endothelial nitric oxide synthase in vascular 
endothelial growth factor-induced angiogenesis and vascular permeability. Proc Natl Acad Sci U S A. 2001;98(5):2604-2609.

37. Pober JS, Sessa WC. Evolving functions of endothelial cells in inflammation. Nat Rev Immunol. 2007;7(10):803-815.

38. Ley K, Laudanna C, Cybulsky MI, Nourshargh S. Getting to the site of inflammation: the leukocyte adhesion cascade updated. Nat Rev Immunol. 2007;7(9):678-689.

39. Granger DN, Kubes $P$. The microcirculation and inflammation: modulation of leukocyte-endothelial cell adhesion. J Leukoc Biol. 1994;55(5):662-675.

40. Arndt H, Russell JB, Kurose I, Kubes P, Granger DN. Mediators of leukocyte adhesion in rat mesenteric venules elicited by inhibition of nitric oxide synthesis. Gastroenterology. 1993;105(3):675-680.

41. Akimitsu T, Gute DC, Korthuis RJ. Leukocyte adhesion induced by inhibition of nitric oxide production in skeletal muscle. J Appl Physiol (1985). 1995;78(5):1725-1732.

42. Hossain M, Qadri SM, Liu L. Inhibition of nitric oxide synthesis enhances leukocyte rolling and adhesion in human microvasculature. J Inflamm (Lond).9(1):28.

43. Gaboury J, Woodman RC, Granger DN, Reinhardt P, Kubes P. Nitric oxide prevents leukocyte adherence: role of superoxide. Am J Physiol. 1993;265(3 Pt 2):H862-867.

44. Mitchell DJ, Yu J, Tyml K. Local L-NAME decreases blood flow and increases leukocyte adhesion via CD18. Am J Physiol. 1998;274(4 Pt 2):H1264-1268.

45. Davenpeck KL, Gauthier TW, Lefer AM. Inhibition of endothelial-derived nitric oxide promotes P-selectin expression and actions in the rat microcirculation. Gastroenterology. 1994;107(4):1050-1058.

46. Carreau A, Kieda C, Grillon C. Nitric oxide modulates the expression of endothelial cell adhesion molecules involved in angiogenesis and leukocyte recruitment. Exp Cell Res.317(1):29-41.

47. De Caterina R, Libby P, Peng HB, Thannickal VJ, Rajavashisth TB, Gimbrone MA, Jr., Shin WS, Liao JK. Nitric oxide decreases cytokine-induced endothelial activation. Nitric oxide selectively reduces endothelial expression of adhesion molecules and proinflammatory cytokines. J Clin Invest. 1995;96(1):60-68.

48. Fernandez-Hernando C, Yu J, Davalos A, Prendergast J, Sessa WC. Endothelialspecific overexpression of caveolin-1 accelerates atherosclerosis in apolipoprotein E-deficient mice. Am J Pathol. 2010;177(2):998-1003. 
49. Fernandez-Hernando C, Yu J, Suarez Y, Rahner C, Davalos A, Lasuncion MA, Sessa WC. Genetic evidence supporting a critical role of endothelial caveolin-1 during the progression of atherosclerosis. Cell Metab. 2009;10(1):48-54.

50. Frank PG, Lee H, Park DS, Tandon NN, Scherer PE, Lisanti MP. Genetic ablation of caveolin-1 confers protection against atherosclerosis. Arterioscler Thromb Vasc Biol. 2004;24(1):98-105.

51. Lefer DJ, Jones SP, Girod WG, Baines A, Grisham MB, Cockrell AS, Huang PL, Scalia R. Leukocyte-endothelial cell interactions in nitric oxide synthase-deficient mice. Am J Physiol. 1999;276(6 Pt 2):H1943-1950.

52. Chatzizisis YS, Coskun AU, Jonas M, Edelman ER, Feldman CL, Stone PH. Role of endothelial shear stress in the natural history of coronary atherosclerosis and vascular remodeling: molecular, cellular, and vascular behavior. J Am Coll Cardiol. 2007;49(25):2379-2393.

53. Oguninade O, Kameya GT, Truskey GA. Effect of fluid shear stress on the permeability of the arterial endothelium. Ann Biomed Eng. 2002;30(4):430-446.

54. Kelm M, Schrader J. Control of coronary vascular tone by nitric oxide. Circ Res. 1990;66(6):1561-1575.

55. Woldman YY, Sun J, Zweier JL, Khramtsov VV. Direct chemiluminescence detection of nitric oxide in aqueous solutions using the natural nitric oxide target soluble guanylyl cyclase. Free Radic Biol Med. 2009;47(10):1339-1345.

56. Sato $\mathrm{M}$, Hida $\mathrm{N}$, Umezawa $\mathrm{Y}$. Imaging the nanomolar range of nitric oxide with an amplifier-coupled fluorescent indicator in living cells. Proc Natl Acad Sci U S A. 2005;102(41):14515-14520.

57. Ando J, Komatsuda T, Kamiya A. Cytoplasmic calcium response to fluid shear stress in cultured vascular endothelial cells. In Vitro Cell Dev Biol. 1988;24(9):871-877.

58. Shen J, Luscinskas FW, Connolly A, Dewey CF, Jr., Gimbrone MA, Jr. Fluid shear stress modulates cytosolic free calcium in vascular endothelial cells. Am J Physiol. 1992;262(2 Pt 1):C384-390.

59. Kuchan MJ, Frangos JA. Role of calcium and calmodulin in flow-induced nitric oxide production in endothelial cells. Am J Physiol. 1994;266(3 Pt 1):C628-636.

60. Buga GM, Gold ME, Fukuto JM, Ignarro LJ. Shear stress-induced release of nitric oxide from endothelial cells grown on beads. Hypertension. 1991;17(2):187-193.

61. O'Neill WC. Flow-mediated NO release from endothelial cells is independent of $\mathrm{K}+$ channel activation or intracellular Ca2+. Am J Physiol. 1995;269(4 Pt 1):C863-869. 
62. Bates DO, Curry FE. Vascular endothelial growth factor increases microvascular permeability via a $\mathrm{Ca}(2+)$-dependent pathway. Am J Physiol. 1997;273(2 Pt 2):H687-694.

63. He P, Curry FE. Depolarization modulates endothelial cell calcium influx and microvessel permeability. Am J Physiol. 1991;261(4 Pt 2):H1246-1254.

64. Norwood N, Moore TM, Dean DA, Bhattacharjee R, Li M, Stevens T. Store-operated calcium entry and increased endothelial cell permeability. Am J Physiol Lung Cell Mol Physiol. 2000;279(5):L815-824.

65. Mayhan WG, Joyner WL. The effect of altering the external calcium concentration and a calcium channel blocker, verapamil, on microvascular leaky sites and dextran clearance in the hamster cheek pouch. Microvasc Res. 1984;28(2):159-179.

66. Wiesner TF, Berk BC, Nerem RM. A mathematical model of the cytosolic-free calcium response in endothelial cells to fluid shear stress. Proc Natl Acad Sci U S A. 1997;94(8):3726-3731.

67. Yamamoto K, Korenaga R, Kamiya A, Ando J. Fluid shear stress activates $\mathrm{Ca}(2+)$ influx into human endothelial cells via P2X4 purinoceptors. Circ Res. 2000;87(5):385-391.

68. Goligorsky MS. Mechanical stimulation induces $\mathrm{Ca} 2+i$ transients and membrane depolarization in cultured endothelial cells. Effects on $\mathrm{Ca} 2+\mathrm{i}$ in co-perfused smooth muscle cells. FEBS Lett. 1988;240(1-2):59-64.

69. Mo M, Eskin SG, Schilling WP. Flow-induced changes in $\mathrm{Ca} 2+$ signaling of vascular endothelial cells: effect of shear stress and ATP. Am J Physiol. 1991;260(5 Pt 2):H1698-1707.

70. Dull RO, Tarbell JM, Davies PF. Mechanisms of flow-mediated signal transduction in endothelial cells: kinetics of ATP surface concentrations. J Vasc Res. 1992;29(6):410-419.

71. Dull RO, Davies PF. Flow modulation of agonist (ATP)-response $(\mathrm{Ca} 2+)$ coupling in vascular endothelial cells. Am J Physiol. 1991;261(1 Pt 2):H149-154.

72. Sill HW, Chang YS, Artman JR, Frangos JA, Hollis TM, Tarbell JM. Shear stress increases hydraulic conductivity of cultured endothelial monolayers. Am J Physiol. 1995;268(2 Pt 2):H535-543.

73. Chang YS, Yaccino JA, Lakshminarayanan S, Frangos JA, Tarbell JM. Shearinduced increase in hydraulic conductivity in endothelial cells is mediated by a nitric oxide-dependent mechanism. Arterioscler Thromb Vasc Biol. 2000;20(1):35-42. 
74. DePaola N, Phelps JE, Florez L, Keese CR, Minnear FL, Giaever I, Vincent P. Electrical impedance of cultured endothelium under fluid flow. Ann Biomed Eng. 2001;29(8):648-656.

75. Seebach J, Donnert G, Kronstein R, Werth S, Wojciak-Stothard B, Falzarano D, Mrowietz C, Hell SW, Schnittler HJ. Regulation of endothelial barrier function during flow-induced conversion to an arterial phenotype. Cardiovasc Res. 2007;75(3):596607.

76. Tarbell JM. Shear stress and the endothelial transport barrier. Cardiovasc Res.87(2):320-330.

77. Curry FR, Adamson RH. Vascular permeability modulation at the cell, microvessel, or whole organ level: towards closing gaps in our knowledge. Cardiovasc Res. 2010;87(2):218-229.

78. Montermini D, Winlove CP, Michel C. Effects of perfusion rate on permeability of frog and rat mesenteric microvessels to sodium fluorescein. J Physiol. 2002;543(Pt 3):959-975.

79. Kajimura M, Michel $\mathrm{CC}$. Flow modulates the transport of $\mathrm{K}+$ through the walls of single perfused mesenteric venules in anaesthetised rats. J Physiol. 1999;521 Pt 3:665-677.

80. Neal CR, Bates DO. Measurement of hydraulic conductivity of single perfused Rana mesenteric microvessels between periods of controlled shear stress. J Physiol. 2002;543(Pt 3):947-957.

81. Williams DA. A shear stress component to the modulation of capillary hydraulic conductivity (Lp). Microcirculation. 1996;3(2):229-232.

82. Adamson RH, Sarai RK, Altangerel A, Clark JF, Weinbaum S, Curry FE. Microvascular permeability to water is independent of shear stress, but dependent on flow direction. Am J Physiol Heart Circ Physiol. 2013;304(8):H1077-1084.

83. Williams DA. Network assessment of capillary hydraulic conductivity after abrupt changes in fluid shear stress. Microvasc Res. 1999;57(2):107-117.

84. Kim MH, Harris NR, Tarbell JM. Regulation of capillary hydraulic conductivity in response to an acute change in shear. Am J Physiol Heart Circ Physiol. 2005;289(5):H2126-2135.

85. Olesen SP, Clapham DE, Davies PF. Haemodynamic shear stress activates a K+ current in vascular endothelial cells. Nature. 1988;331(6152):168-170. 
86. Gudi SR, Clark CB, Frangos JA. Fluid flow rapidly activates $\mathrm{G}$ proteins in human endothelial cells. Involvement of $G$ proteins in mechanochemical signal transduction. Circ Res. 1996;79(4):834-839.

87. Rizzo V, Morton C, DePaola N, Schnitzer JE, Davies PF. Recruitment of endothelial caveolae into mechanotransduction pathways by flow conditioning in vitro. $\mathrm{Am} \mathrm{J}$ Physiol Heart Circ Physiol. 2003;285(4):H1720-1729.

88. Osawa M, Masuda M, Kusano K, Fujiwara K. Evidence for a role of platelet endothelial cell adhesion molecule-1 in endothelial cell mechanosignal transduction: is it a mechanoresponsive molecule? J Cell Biol. 2002;158(4):773-785.

89. Tarbell JM, Pahakis MY. Mechanotransduction and the glycocalyx. $J$ Intern Med. 2006;259(4):339-350.

90. Florian JA, Kosky JR, Ainslie K, Pang Z, Dull RO, Tarbell JM. Heparan sulfate proteoglycan is a mechanosensor on endothelial cells. Circ Res. 2003;93(10):e136142.

91. Barakat Al. Dragging along: the glycocalyx and vascular endothelial cell mechanotransduction. Circ Res. 2008;102(7):747-748.

92. Adamson RH. Permeability of frog mesenteric capillaries after partial pronase digestion of the endothelial glycocalyx. J Physiol. 1990;428:1-13.

93. Yuan $D, X u S$, He $P$. Enhanced permeability responses to inflammation in streptozotocin-induced diabetic rat venules: Rho-mediated alterations of actin cytoskeleton and VE-cadherin. Am J Physiol Heart Circ Physiol. 2014;307(1):H4453.

94. Weinbaum S, Zhang $X$, Han $\mathrm{Y}$, Vink H, Cowin SC. Mechanotransduction and flow across the endothelial glycocalyx. Proc Natl Acad Sci U S A. 2003;100(13):79887995.

95. Osborn EA, Rabodzey A, Dewey CF, Jr., Hartwig JH. Endothelial actin cytoskeleton remodeling during mechanostimulation with fluid shear stress. Am J Physiol Cell Physiol. 2006;290(2):C444-452.

96. Thi MM, Tarbell JM, Weinbaum S, Spray DC. The role of the glycocalyx in reorganization of the actin cytoskeleton under fluid shear stress: a "bumper-car" model. Proc Natl Acad Sci U S A. 2004;101(47):16483-16488.

97. Chiu JJ, Chen LJ, Lee PL, Lee Cl, Lo LW, Usami S, Chien S. Shear stress inhibits adhesion molecule expression in vascular endothelial cells induced by coculture with smooth muscle cells. Blood. 2003;101(7):2667-2674. 
98. Morigi M, Zoja C, Figliuzzi M, Foppolo M, Micheletti G, Bontempelli M, Saronni M, Remuzzi G, Remuzzi A. Fluid shear stress modulates surface expression of adhesion molecules by endothelial cells. Blood. 1995;85(7):1696-1703.

99. Lopez-Quintero SV, Amaya R, Pahakis M, Tarbell JM. The endothelial glycocalyx mediates shear-induced changes in hydraulic conductivity. Am J Physiol Heart Circ Physiol. 2009;296(5):H1451-1456.

100. Chen Y, Chen H, Liu X, Lei S, Mao Y, Zhang W. [Effect of flow shear stress on the expression of adhesion molecules of endothelial cells]. Sheng Wu Yi Xue Gong Cheng Xue Za Zhi. 2001;18(2):201-205.

101. Nagel T, Resnick N, Atkinson WJ, Dewey CF, Jr., Gimbrone MA, Jr. Shear stress selectively upregulates intercellular adhesion molecule-1 expression in cultured human vascular endothelial cells. J Clin Invest. 1994;94(2):885-891.

102. Granger DN, Kubes P. Nitric oxide as antiinflammatory agent. Methods Enzymol. 1996;269:434-442.

103. Zhou X, He P. Endothelial [Ca2+]i and caveolin-1 antagonistically regulate eNOS activity and microvessel permeability in rat venules. Cardiovasc Res.87(2):340-347.

104. Tarbell JM, Ebong EE. The endothelial glycocalyx: a mechano-sensor and transducer. Sci Signal. 2008;1(40):pt8.

105. Pahakis MY, Kosky JR, Dull RO, Tarbell JM. The role of endothelial glycocalyx components in mechanotransduction of fluid shear stress. Biochem Biophys Res Commun. 2007;355(1):228-233. 


\title{
CHAPTER 2
}

\section{CAVEOLIN-1 SCAFFOLDING DOMAIN PROMOTES LEUKOCYTE ADHESION BY REDUCED BASAL ENDOTHELIAL NITRIC OXIDE-MEDIATED ICAM-1 PHOSPHORYLATION IN RAT MESENTERIC VENULES}

\author{
Sulei Xu, Xueping Zhou, Dong Yuan, Yanchun Xu, and Pingnian He \\ Department of Physiology and Pharmacology, School of Medicine, West Virginia \\ University, Morgantown, WV 26506
}

Running Title: Reduced NO by applied caveolin-1 promotes leukocyte adhesion

This chapter was published in American Journal of Physiology - Heart and Circulatory Physiology. Xu, S, Zhou, X, Yuan, D, Xu, Y and He, P. Caveolin-1 scaffolding domain promotes leukocyte adhesion by reduced basal endothelial nitric oxide-mediated ICAM-1 phosphorylation in rat mesenteric venules. Am J Physiol Heart Circ Physiol. 2013, 305(10):H1484-93 Copyright $@ 2013$, American Journal of Physiology - Heart and Circulatory 


\begin{abstract}
Exogenously applied caveolin-1 scaffolding domain (CAV) has been shown to inhibit inflammatory mediator-induced nitric oxide (NO) production and NO-mediated increases in microvessel permeability. However, the effect of CAV on endothelial basal NO that prevents leukocyte adhesion remains unknown. This study aims to investigate the roles of exogenously applied CAV in endothelial basal NO production, leukocyte adhesion, and adhesion-induced changes in microvessel permeability. Experiments were conducted in individually perfused rat mesenteric venules. Microvessel permeability was determined by measuring hydraulic conductivity (Lp). NO was quantified with fluorescence imaging in DAF-2-loaded vessels. Perfusing venules with CAV inhibited basal NO production without affecting basal Lp. Resuming blood flow in CAV perfused vessels significantly increased leukocyte adhesion. The firmly adherent leukocytes altered neither basal Lp, nor adherens junction integrity. Increases in Lp occurred only upon formyl-Met-Leu-Phe application that induces release of reactive oxygen species from the adherent leukocytes. The application of NO synthase inhibitor showed similar results to CAV, and NO donor abolished CAVmediated leukocyte adhesion. Immunofluorescence staining showed increases in binding of ICAM-1 to an adhesion-blocking antibody concurrent with a Src-dependent ICAM-1 phosphorylation following CAV perfusion. Pre-perfusing vessels with anti-ICAM-1 blocking antibody or a Src kinase inhibitor attenuated CAV-induced leukocyte adhesion. These results indicate that the application of CAV, in addition to preventing excessive NOmediated permeability increases, also causes reduction of basal NO and promotes ICAM1-mediated leukocyte adhesion through Src activation-mediated ICAM-1 phosphorylation. CAV-induced leukocyte adhesion was uncoupled from leukocyte oxidative burst and microvessel barrier function, unless in the presence of a secondary stimulation.
\end{abstract}


Key Words: caveolin-1, microvessel permeability, leukocyte adhesion, reactive oxygen species ICAM-1 phorsphorylation 


\section{INTRODUCTION}

Nitric oxide (NO), in addition to being a potent vasodilator, has also been considered a "double edged sword"-mediator in inflammation. Excessive NO production induced by inflammatory mediators contributes to an increase in microvessel permeability and plays a pro-inflammatory role. ${ }^{1-3}$ Constitutive (or basal) NO production, on the other hand, has been reported to prevent leukocyte adhesion and platelet aggregation on the vessel wall, therefore, playing an anti-inflammatory role. ${ }^{4-6}$

Caveolin-1, the major structural protein in the caveolae of endothelial cells, is a recognized endogenous inhibitor of endothelial nitric oxide synthase (eNOS). ${ }^{7,8}$ The discovery of the scaffolding domain of caveolin-1 (CAV) that directly interacts with eNOS, and the use of Antennapedia homeodomain (AP) fusions to facilitate CAV uptake by living cells, provided a valuable tool for investigating the mechanisms involved in the regulation of eNOS activity through protein-protein interaction and NO-dependent vascular functions in vivo. Our previous studies demonstrated that exogenously applying AP-CAV to a perfused intact microvessel inhibits platelet activating factor (PAF)-induced NO production and NO-mediated increases in microvessel permeability. ${ }^{9,10}$ In whole animal studies, the administration of AP-CAV suppressed tissue edema and vascular leakage. ${ }^{11}$ These studies suggest an anti-inflammatory therapeutic potential for AP-CAV. However, whether the exogenously applied caveolin-1 peptide affects basal endothelial NO production has not been studied. If AP-CAV inhibits basal NO, whether it causes leukocyte adhesion and increases microvessel permeability is unknown. This study directly addresses these 
questions by measuring basal NO, microvessel permeability, and leukocyte adhesion in AP-CAV-perfused intact microvessels.

Experiments were conducted in individually perfused venular microvessels in rat mesenteries. We first quantitatively measured the effect of AP-CAV on endothelial basal NO production with DAF-2 using fluorescence imaging. We then examined the effect of AP-CAV on leukocyte adhesion, the mechanisms involved in the increases in adhesive activity of endothelial cells, and their impact on microvessel permeability. Permeability was determined by measuring hydraulic conductivity (Lp). The changes in endothelial junction integrity and adhesive capacity of adhesion molecules were evaluated by confocal imaging of immunofluorescence staining on AP-CAV-perfused microvessels. 


\section{METHODS}

\section{Animal preparation}

Experiments were carried out on venular microvessels in rat mesenteries. All procedures and animal use were approved by the Animal Care and Use Committee at West Virginia University. Female Sprague-Dawley rats (2-3-mo old; body wt 220-250 g; Hilltop Laboratory Animals; Scottdale, PA) were anesthetized with pentobarbital sodium given subcutaneously. The initial dosage was $65 \mathrm{mg} / \mathrm{kg}$ of body weight with an additional $3 \mathrm{mg}$ dose given as needed. The trachea was intubated, and a midline surgical incision $(1.5-2 \mathrm{~cm})$ was made in the abdominal wall. The mesentery was gently moved out of the abdominal cavity and spread over a glass cover slip attached to an animal tray. The upper surface of the mesentery was continuously superfused with mammalian Ringer solution. The temperature of the superfusate was maintained at $37^{\circ} \mathrm{C}$. All experiments were carried out on venular microvessels with diameters ranging between 40 and $50 \mu \mathrm{m}$. Each experiment was performed on a single microvessel with one experiment per animal.

\section{Measurement of $L_{p}$ in individually perfused rat mesenteric microvessels}

Modified Landis technique was used to measure the volume flux of water across the microvessel wall. The methods have been evaluated in detail. ${ }^{12,} 13$ In brief, a single microvessel was cannulated with a micropipette and perfused with albumin-Ringer solution (control) containing 1\% (vol/vol) hamster red blood cells as markers. A hydrostatic pressure (range 40-60 $\mathrm{cmH}_{2} \mathrm{O}$ ) was applied through the micropipette to the microvessel lumen to maintain a continuous flow. Water flux was measured when the vessel was briefly occluded downstream. Lp was calculated as the slope of the relationship between the initial water flux 
per unit area of microvessel wall and the pressure difference across the microvessel wall. In each experiment, the baseline $L p$ and the $L p$ after application of testing solutions were measured in the same vessel and the changes in $L p$ were expressed as the ratio of $L p_{\text {test }} /$

Lp control

\section{Fluorescence imaging of endothelial nitric oxide production}

Endothelial NO production was measured on DAF-2 DA loaded vessels using fluorescence imaging. Experiments were performed on a Nikon Diaphod 300 microscope equipped with a 12-bit digital CCD camera (ORCA; Hamamatsu) and a computer controlled shutter (Lambda 10-2; Sutter Instrument; Novato, CA). A 75-W xenon lamp was used as the light source. The excitation wavelength for DAF-2 was selected by an interference filter $(480 / 40 \mathrm{~nm})$, and emission was separated by a dichroic mirror $(505 \mathrm{~nm})$ and a band-pass barrier (535/50 nM). All the images were acquired and analyzed using Metafluor software (Universal Imaging). To minimize the photo-bleaching, a neutral density filter (0.5 ND) was positioned in front of the interference filter and the exposure time was minimized to 0.12 $\sec$ at 1 min intervals.

During the experiments, each vessel was cannulated and perfused with albumin-Ringer solution containing DAF-2 DA $(5 \mu \mathrm{M})$ throughout the experimental duration. Image collection was started at the beginning of DAF-2 DA perfusion. All images were collected from a group of endothelial cells located in the same focal plane of the vessel wall using a Nikon Fluor lens (x20, numerical aperture, 0.75). A steady-state of endothelial DAF-2 fluorescence (FIDAF) with continuous perfusion of DAF-2 DA was indicated by the rate change of the increased $\mathrm{FI}$. Quantitative analysis was conducted at the individual 
endothelial cell level using selected regions of interest (ROIs) along the vessel wall. Each $\mathrm{ROI}$ covers the area of one individual cell as indicated by the fluorescence outline. The tissue autofluorescence was subtracted from all of the measured Fls. The basal NO production rate was calculated from the slope of the FlDAF increase during albumin-Ringer perfusion after DAF-2 loading reached the steady-state. FlDAF was expressed in arbitrary units (AU). The rate of FlDAF increase (df/dt) derived by first differential conversion of cumulative FlDAF over time indicates the rate of NO production. Details have been described. ${ }^{14}$

\section{Immunofluorescence staining and confocal imaging}

VE-cadherin and adherent leukocyte staining was conducted in vessels after CAVinduced leukocyte adhesion. Phosphorylated ICAM-1 at Y526 staining was conducted in vessels after 30 min AP-CAV perfusion in the presence or absence of the Src kinase inhibitor, PP1. The rat mesentery bearing the perfused vessel was fixed with $1 \%$ paraformaldehyde and treated with triton X-100 before exposure to primary antibodies against VE-cadherin (C-19, sc-6458, Santa Cruz Biotechnology) and CD45 (clone OX-1, Biolegend), or phospho-(Y526)-ICAM-1 (ab51033, Abcam) and followed by incubation with their corresponding secondary antibodies (Invitrogen).

ICAM-1 staining was conducted in intact microvessels. Each venule was first perfused with albumin-Ringer solution for $10 \mathrm{~min}$ and then perfused with ICAM-1 primary antibodies (1A29 BD Pharmingen, $0.1 \mathrm{mg} / \mathrm{ml}$ ) for $20 \mathrm{~min}$. The secondary antibody (Invitrogen) was applied to each vessel for $15 \mathrm{~min}$ after washout of the primary antibody from the vessel lumen. Nuclei were labeled by perfusion vessels with DRAQ5 (Biostatus). The confocal 
images were acquired after all of the fluorescence markers were washed out from the vessel lumen.

All of the images were acquired using Leica confocal microscope. Each image stack was collected by optical sectioning at successive $X-Y$ focal planes with a vertical depth of $0.3 \mu \mathrm{m}$ (phospho-(Y526)-ICAM-1) and $0.5 \mu \mathrm{m}$ (ICAM-1) using Leica X25 objective (HC Plan APO, NA 0.95) and 1024 x 1024 scanning format. Leica software was used for image acquisition and image analysis.

The fluorescence intensity (FI) of labeled ICAM-1 and phospho-(Y526)-ICAM-1 was quantified from a segment of the vessel wall. The total FI was calculated as area $\times$ depth $\times$ mean amplitude, where the area is the selected ROI per vessel section and the depth is the total number of images at $z$ dimension. Because ICAM-1 was expressed on endothelial cell surface, $\mathrm{Fl}$ was quantified as total intensity per square micrometer of vessel wall (FI/A). Assuming a cylindrical geometry, surface area of the vessel wall was calculated as $2 \pi \times r$ $\times L$, where $r$ is the radius of the microvessel and $L$ is the length of selected $R O I$ from the vessel.

\section{Solutions and reagents}

Mammalian Ringer solution ${ }^{15}$ was used for the experiments. All perfusates contained albumin-Ringer solution (BSA, $10 \mathrm{mg} / \mathrm{ml}$ ). AP-CAV, the fusion peptide of caveolin-1 scaffolding domain with AP, the Antennapedia internalization sequence from Drosophila Antennapedia homeodomain, and AP-CAV-X, the fusion peptide of scrambled CAV with AP were synthesized by Tufts University. ${ }^{11}$ The chemotactic peptide formyl-Met-Leu-Phe$\mathrm{OH}$ (fMLP) was purchased from Calbiochem (San Diego, CA). PP1 (4-amino-5-(4- 
methylphenyl)-7-(t-butyl)pyrazolo[3,4-d]pyrimidine), sodium nitroprusside and $\mathrm{N}$ (omega)monomethyl-I-arginine (L-NMMA) were purchased from Sigma (St. Louis, MO). The stock solutions were prepared with $100 \%$ DMSO and each final solution was achieved by $>1: 1,000$ dilution with albumin-Ringer solution.

\section{Data analysis and statistics}

All values are mean \pm SE. Paired t-test was used for paired data analysis. ANOVA was used to compare data between groups. A probability value of $P<0.05$ was considered statistically significant. 


\section{RESULTS}

\section{AP-CAV inhibits basal NO production without affecting basal Lp in intact venules}

The effect of AP-CAV on basal NO was examined in four vessels. A steady-state of DAF2 fluorescence intensity, FlDAF, was reached at $39.5 \pm 1.3$ min with continuous DAF-2 DA perfusion. The FlDAF accumulation rate, an indication of basal NO production rate, was 0.13 $\pm 0.01 \mathrm{AU}$ per min. After perfusion of each vessel with AP-CAV $(10 \mu \mathrm{M})$, FlDAF accumulation rate significantly decreased to $0.02 \pm 0.01 \mathrm{AU}$ per min within $1 \mathrm{~min}(P<0.01)$. To confirm that DAF-2 was still functional after the application of AP-CAV, a NO donor, SNP (10 $\mu \mathrm{M})$ was added to the perfusate in the presence of AP-CAV in two of the vessels. The FlDAF increased at a relatively linear rate of $0.15 \pm 0.01 \mathrm{AU}$ per min, which is comparable with that under control conditions (Fig. 1A and 1C). Based on these results we estimate that the level of NO generated by $10 \mu \mathrm{M}$ SNP is close to basal NO production rate. To compare the inhibitory effect of AP-CAV on basal NO with that of the NOS inhibitor, basal NO production was measured in another eight vessels in the absence or presence of L-NMMA ( $500 \mu \mathrm{M}$, four vessels per group). The mean FlDAF accumulation rate of four vessels was $0.13 \pm 0.02$ $\mathrm{AU}$ per min and was significantly decreased to $0.01 \pm 0.002 \mathrm{AU}$ per min in the presence of L-NMMA (Fig. 1B and 1C), demonstrating a similar magnitude of basal NO reduction to that of AP-CAV.

To examine whether reduced basal NO via perfusion of AP-CAV has a direct effect on basal permeability, we measured $L p$ in four microvessels. The mean baseline $L p$ of the four vessels was $1.8 \pm 0.2 \times 10^{-7} \mathrm{~cm} \cdot \mathrm{s}^{-1} \cdot \mathrm{cmH}_{2} \mathrm{O}^{-1}$. After perfusing vessels with AP-CAV $(10 \mu \mathrm{M})$ for $30 \mathrm{~min}$, the mean $\mathrm{Lp}$ was $1.8 \pm 0.3 \times 10^{-7} \mathrm{~cm} \cdot \mathrm{s}^{-1} \cdot \mathrm{cmH}_{2} \mathrm{O}^{-1}$, not significantly different from 
that of the control. Fig. 2 shows a single experiment and the data summary. Our previous study demonstrated that perfusion of mesenteric venules with AP-CAV at $1 \mu \mathrm{M}$ for 30 min also did not affect basal Lp. ${ }^{9}$

\section{AP-CAV perfusion induces leukocyte adhesion without increasing microvessel permeability unless in the presence of a secondary stimulation.}

The effect of AP-CAV-induced basal NO reduction on leukocyte adhesion was examined in 8 microvessels. Each vessel was perfused with 1 or $10 \mu \mathrm{M}$ of AP-CAV for 30 min followed by resuming blood flow in the perfused microvessel for $10 \mathrm{~min}$. When each vessel was re-cannulated with albumin-Ringer perfusate, we observed a dose-dependent leukocyte adhesion. In vessels perfused with AP-CAV at $1 \mu \mathrm{M}$, the adherent leukocytes increased from baseline levels of $0.7 \pm 0.1$ to $8.4 \pm 0.1$ per $100 \mu \mathrm{m}$ of vessel length $(n=3$, $p<0.05)$. In $10 \mu \mathrm{M}$ AP-CAV-perfused vessels, the mean adherent leukocytes increased from baseline levels of $1.3 \pm 0.2$ to $26.3 \pm 3.2$ per $100 \mu \mathrm{m}$ of vessel length $(n=5)$. The left panel of Fig. 3A demonstrates the representative images of leukocyte adhesion before and after AP-CAV $(10 \mu \mathrm{M})$ perfusion followed by resumed blood flow from a single vessel. When Lp was measured in the presence of CAV-induced adherent leukocytes, the mean value was $2.1 \pm 0.1 \times 10^{-7} \mathrm{~cm} \cdot \mathrm{s}^{-1} \cdot \mathrm{cmH}_{2} \mathrm{O}^{-1}$, which was not significantly different from the mean baseline Lp, $1.7 \pm 0.2 \times 10^{-7} \mathrm{~cm} \cdot \mathrm{s}^{-1} \cdot \mathrm{cmH}_{2} \mathrm{O}^{-1}$.

Our previous study showed that formyl-Met-Leu-Phe (fMLP) can stimulate reactive oxygen species (ROS) release from TNF-a-induced adherent leukocytes, resulting in increases in microvessel Lp. ${ }^{16}$ Here, we further investigated whether exposing CAVinduced adherent leukocytes to fMLP increases Lp. We added fMLP $(10 \mu M)$ to the 
perfusate after leukocyte adhesion in AP-CAV perfused vessels. To maximize the local concentration of ROS released from the adherent leukocytes upon FMLP stimulation, the perfusate was kept at a balanced pressure (no flow) for 5 min before Lp measurement. Lp measured after 5 min stationary flow transiently increased to mean peak values of $4.8 \pm$ 0.6 and $16.3 \pm 2.1$ times that of the control in 1 and $10 \mu \mathrm{M}$ AP-CAV perfused vessels, respectively. The 5 min stationary flow in the absence of adherent leukocytes does not affect Lp. ${ }^{16}$ Fig 3B shows a typical time course of the Lp changes in a single experiment. Fig. $3 \mathrm{C}$ shows result summary (2 panels on the left).

\section{Nitric oxide donor prevents AP-CAV induced leukocyte adhesion}

To examine whether the AP-CAV-induced reduction of basal NO is responsible for increased leukocyte adhesion, we applied a NO donor, SNP, during AP-CAV perfusion in four vessels. The mean baseline $\mathrm{Lp}$ was $1.7 \pm 0.1 \times 10^{-7} \mathrm{~cm} \cdot \mathrm{s}^{-1} \cdot \mathrm{cmH}_{2} \mathrm{O}^{-1}$. Each vessel was first perfused with AP-CAV $(10 \mu \mathrm{M})$ and SNP $(10 \mu \mathrm{M})$ for $30 \mathrm{~min}$, followed by $10 \mathrm{~min}$ of resumed blood flow with superfusate containing SNP $(20 \mu \mathrm{M})$. When each vessel was recannulated with albumin-Ringer perfusate, the mean number of adherent leukocytes was only $3.7 \pm 1.1$ per $100 \mu \mathrm{m}$ of vessel length, which was a significant reduction from AP-CAV perfusion alone and not significantly different from that of the baseline. The right panel of Fig. 3A demonstrates the representative images before and after AP-CAV and SNP perfusion followed by resumed blood flow from one single vessel. We also measured Lp when vessels were perfused with SNP and AP-CAV, which showed no significant difference from that of the control (Fig. 3C, 3rd panel from left).

Scrambled AP-CAV peptide does not affect leukocyte adhesion and microvessel Lp 
AP-CAV-X $(10 \mu \mathrm{M})$ was used to examine the specificity of AP-CAV in another three microvessels. Experiments were conducted under identical conditions to that of AP-CAV application. No significant leukocyte adhesion or changes in Lp were observed after resuming blood flow in AP-CAV-X perfused vessels. The mean number of adherent leukocytes before and after resuming blood flow was $0.8 \pm 0.1$ and $1.1 \pm 0.2$ / $100 \mu \mathrm{m}$ of vessel length, and the mean $L p$ measured under each condition was $1.7 \pm 0.2$ and $1.8 \pm$ $0.2 \times{ }^{-7} \mathrm{~cm} \cdot \mathrm{s}^{-1} \cdot \mathrm{cmH}_{2} \mathrm{O}^{-1}$, respectively (Fig. $3 \mathrm{C}$, far right panel).

\section{Inhibition of NOS by L-NMMA shows similar results to the application of AP-CAV}

If AP-CAV-induced leukocyte adhesion is attributed to its inhibitory effect on basal NO, we would expect the similar effect from the application of $\mathrm{N}^{\mathrm{G}}-\mathrm{Methyl}-\mathrm{L}-\operatorname{arginine}(\mathrm{L}-\mathrm{NMMA}$ ), a NOS inhibitor. Experiments were conducted in three microvessels. Fig. 4A shows a representative time course of the $L p$ changes in a single experiment. The mean control Lp of three vessels was $2.4 \pm 0.3 \times 10^{-7} \mathrm{~cm} \cdot \mathrm{s}^{-1} \cdot \mathrm{cmH}_{2} \mathrm{O}^{-1}$. After application of L-NMMA to both perfusate $(100 \mu \mathrm{M})$ and superfusate $(500 \mu \mathrm{M})$ for 30 minutes followed by 20 minutes resumed blood flow, the mean adherent leukocytes to the microvessel wall increased from a mean baseline level of $0.8 \pm 0.2$ to $14.8 \pm 2.5$ per $100 \mu \mathrm{m}$ of vessel length. Consistent with the results of AP-CAV-induced leukocyte adhesion, Lp measured in the presence of L-NMMA-induced adherent leukocytes was $2.7 \pm 0.6 \times 10^{-7} \mathrm{~cm} \cdot \mathrm{s}^{-1} \cdot \mathrm{cmH}_{2} \mathrm{O}^{-1}$, not significantly different from its control. When fMLP was applied to the perfusate, Lp measured after 5 min of stationary flow transiently increased to a mean peak value of $5.4 \pm 0.9$ times that of the control. Fig. 4B summarizes the results. 


\section{AP-CAV-induced leukocyte adhesion does not change endothelial adherens junction}

Our results showed that AP-CAV-induced leukocyte adhesion did not change basal Lp. We then further examined the location of adherent leukocytes and whether the adherent leukocytes cause local structural changes of endothelial junctions. Immunofluorescence staining was used to simultaneously label adherent leukocytes and VE-cadherin in three vessels. The representative confocal images shown in Fig. 5 illustrate that AP-CAVinduced leukocyte adhesion did not change the distributions of VE-cadherin at endothelial junctions. Endothelial junctions were outlined by continuous VE-cadherin that had no apparent interruptions, even underneath the site of the attached leukocyte. In addition, the dual staining of both leukocyte and VE-cadherin demonstrated that the majority of adherent leukocytes selectively adhered at or near endothelial junctions.

\section{Reduced basal NO by AP-CAV increases the adhesive capacity of ICAM-1 on the surface of endothelium lining microvessel walls.}

Our results showed that leukocytes were firmly attached to the venular walls after resuming blood flow in vessels that has been exposed to AP-CAV. We then investigated whether AP-CAV-induced NO reduction affects the adhesive capacity of intercellular adhesion molecule-1 (ICAM-1), an important molecule mediating the firm adhesion of leukocytes to the vascular endothelium. A monoclonal blocking antibody directed against ICAM-1 (mAb1A29) ${ }^{17,} 18$ was used to evaluate the changes in adhesive activity of ICAM-1 on endothelial cells lining the venular walls after 30 min perfusion of AP-CAV. AP-CAV perfusion at 1 and $10 \mu \mathrm{M}$ significantly increased the mean $\mathrm{FI}$ of mAb1A29 to $2.4 \pm 0.2$ and 
$9.5 \pm 1.7$ times that of the control, respectively ( $n=4$ per group). The application of $10 \mu \mathrm{M}$ SNP abolished AP-CAV-induced increases in mAb1A29 binding $(n=3)$, and perfusion of AP-CAV-X $(10 \mu M)$ showed no difference in mAb1A29 binding from that of the control $(n=$ 3). Fig. 6 shows representative confocal images and summarized FI quantification of mAb1A29 staining in 5 group of studies.

\section{Anti-ICAM-1 blocking antibody attenuates AP-CAV-induced leukocyte adhesion}

To confirm that the specific binding of mAb1A29 to ICAM-1 are directly associated with the adhesive activity of ICAM-1, we perfused microvessels with mAb1A29 before and during AP-CAV application. The initial number of adherent leukocytes under albuminRinger perfusion was $1.0 \pm 0.3$ per $100 \mu \mathrm{m}$ of vessel length $(n=5)$. Each vessel was then perfused with mAb1A29 $(0.1 \mathrm{mg} / \mathrm{ml})$ for 30 minutes followed by perfusion of AP-CAV (10 $\mu \mathrm{M})$ in the presence of mAb1A29 for another 30 min before resuming blood flow. When each vessel was re-cannulated with albumin-Ringer perfusate after 10 min of blood flow, the mean number of adherent leukocytes was only $4.5 \pm 0.8$ per $100 \mu \mathrm{m}$ of vessel length, which is a significant reduction from that of AP-CAV perfusion alone $(26.3 \pm 3.2$ per 100 $\mu \mathrm{m}$ of vessel length). Fig. $6 \mathrm{C}$ summarizes the results.

\section{ICAM-1 Phosphorylation at Tyrosine 526 is required for AP-CAV-induced leukocyte adhesion}

Studies have demonstrated that TNF- $\alpha$-induced early phase of leukocyte adhesion involves Src activation-mediated phosphorylation of ICAM-1. 19, 20 We then examined whether this mechanism also applies to AP-CAV-induced ICAM-1-dependent leukocyte 
adhesion using fluorescence immunostaining and confocal imaging. We found that $30 \mathrm{~min}$ perfusion of AP-CAV $(10 \mu \mathrm{M})$ caused a marked increase in ICAM-1 phosphorylation at tyrosine 526 (Y526) on endothelial cells lining the venular walls compared to that of the control. The same period of AP-CAV-X perfusion showed no such effect. Preferfusing vessels with PP1 $(10 \mu \mathrm{M})$, a src kinase inhibitor, abolished AP-CAV-induced ICAM-1 phosphorylation. Representative confocal images are shown in Fig 7A and the FI quantifications are summarized in Fig 7B ( $n=3$ per group). The role of AP-CAV-induced ICAM-1 phosphorylation in AP-CAV-induced leukocyte adhesion was examined in another 3 vessels. Each vessel was first perfused with PP1 (10 $\mu \mathrm{M})$ for 30 min before adding APCAV $(10 \mu \mathrm{M})$ for another 30 min perfusion. After 10 min of resumed blood flow, the mean number of adherent leukocytes was only $4.6 \pm 0.1$ per $100 \mu \mathrm{m}$ of vessel length, which was significantly lower than that of AP-CAV perfusion alone $(26.3 \pm 3.2$ per $100 \mu \mathrm{m}$ of vessel length). Fig. 7C summarizes the results. 


\section{DISCUSSION}

Our study demonstrated for the first time that exogenously applied AP-CAV, in addition to inhibition of stimulus-induced increases in NO production and microvessel permeability, 9, 10, 21 also reduces basal NO production in endothelial cells lining microvessel walls. Most importantly, this study revealed how reduced basal NO affects the adhesiveness and barrier function of endothelial cells in intact microvessels. While reduced basal NO by APCAV did not directly change basal microvessel permeability, it increased the adhesive capacity of endothelial cells and promotes leukocyte adhesion via Src-mediated phosphorylation of ICAM-1. Our results that NO donor abolished the effect of AP-CAV on increased ICAM-1 binding to a blocking antibody and leukocyte adhesion support an important anti-adhesive role of basal NO in maintaining the homeostasis of vascular walls. Although the experiments focused on the effect of AP-CAV on microvessels, the identified cellular mechanisms may represent the microvascular manifestations associated with reduced basal NO under various pathological conditions in vivo.

\section{CAV reduces basal NO production and promotes leukocyte adhesion in intact venules}

The relationship between Caveolin-1 and eNOS is one of the fundamental discoveries of caveolae involvement in cardiovascular functions. Cell culture and purified protein binding studies demonstrated that caveolin-1 inhibits eNOS activity by a direct interaction with eNOS through its N-terminal "scaffolding domain," located between amino acids 82

and 101 within endothelial plasmalemmal caveolae. ${ }^{7,8,22}$ Although the effect of the caveolin-1 scaffolding domain peptide on eNOS activity has been extensively studied for decades, most of the in vivo studies were conducted in vessels with acute exposure to 
inflammatory mediators. ${ }^{9-11,23}$ We previously demonstrated that when individually perfused venules were exposed to PAF, the excessive NO production triggered by increased endothelial $\left[\mathrm{Ca}^{2+}\right]$ i was a necessary signal for permeability increases, ${ }^{21,} 24$ and that the application of AP-CAV, which inhibits PAF-induced NO, prevents the increases in microvessel permeability. ${ }^{9,10}$ The systemic application of AP-CAV in mice has been shown to suppress carrageenan and mustard oil-induced vascular leakage, as well as the hyperpermeability of tumor vasculature. ${ }^{11,23,25}$ These in vivo evidence suggest a promising anti-inflammatory therapeutic potential of AP-CAV in protecting endothelial barrier function through its inhibitory effect on inflammation-associated eNOS activation. ${ }^{9-11,26}$ However, NO has also been known as an anti-adhesive molecule and plays anti-inflammatory roles in preventing platelet and leukocyte adhesion on microvessel walls. ${ }^{5,27}$ How the inhibitory action of AP-CAV on eNOS affects the anti-inflammatory roles of basal NO has not been previously investigated. Detecting changes in basal NO requires a sensitive method, because the magnitude of basal NO production is much smaller than that induced by inflammatory mediators. Using continuous perfusion of NO indicator, DAF-2, to measure basal NO is a new method recently developed in our laboratory. ${ }^{14}$ This method enables the endothelial cells lining the microvessel wall to maintain a relatively constant dye concentration, which enhances the sensitivity of NO detection. Using this method, we provided direct evidence that the exogenously applied AP-CAV, in addition to its role in suppression of stimulus-induced increases in NO production and microvessel permeability, also inhibits basal NO in intact venules and promotes leukocyte adhesion. The addition of NO donor that reversed the CAV effect on leukocyte adhesion supports a causal relationship between the reduced NO and increased leukocyte adhesion. Based on these 
findings we further investigated the mechanisms involved in increased adhesiveness of microvessel walls by reduced basal NO and the impact of increased adhesive activity of endothelium on microvessel permeability.

Role of basal NO in the regulation of microvessel permeability

NO has been recognized as an important endothelial factor that is not only a potent vasodilator regulating vascular tone and blood pressure, but is also an important intrinsic modulator of microvessel permeability. ${ }^{4,28-31}$ Despite decades of studies, the exact roles of $\mathrm{NO}$ in the regulation of microvessel permeability remain controversial. Some studies showed that the application of NOS inhibitors increased vascular leakages, especially in the presence of blood. ${ }^{4,32-34}$ Others showed that inhibition of NO prevented permeability increases in response to inflammatory mediators. ${ }^{3,9-11,21,23,24,26,31,35}$ The inconsistent results were usually derived from different experimental approaches, organ vasculatures, tissue conditions, and animal species. Our present study was conducted in intact microvessels under basal conditions. Combined with our previous findings on stimuliinduced NO and microvessel permeability, ${ }^{9,10,21,24}$ we revealed dual actions of NO on endothelial functions using the same experimental preparation, but under different conditions, which provides a unified perspective of the roles of NO in the regulation of endothelial function and microvessel permeability. Our results demonstrated that reducing basal NO production by AP-CAV perfusion increased leukocyte adhesion through an alteration of endothelial ICAM-1 adhesive capacity on the surface of endothelial cells lining intact microvessel walls. Neither the increased adhesiveness of ICAM-1 alone, nor ICAM1-mediated leukocyte adhesion directly affect basal microvessel permeability. However, 
the presence of chemokines can trigger a respiratory burst from adherent leukocytes, resulting in ROS-mediated increases in microvessel permeability (Fig 3 and 4). These results demonstrate that the reduced basal NO does not directly increase permeability, but rather primes the microvascular endothelial cells into an adhesive state. These findings reveal the dual roles of NO in the regulation of endothelial functions in microvessels. While basal NO plays anti-adhesive roles in maintaining the homeostasis of vascular walls, the inflammatory mediator-induced excessive NO mediates increases in microvessel permeability.

The relationship between reduced basal NO, ICAM-1 expression, leukocyte adhesion, and microvessel permeability.

Leukocyte adhesion to microvessel walls has been characterized as a series of coordinated interactions mediated by different adhesion molecules. During acute inflammation, the rapid recruitment of leukocytes (minutes) usually involves P-selectin translocation and the secretion of chemokines, ${ }^{36,37}$ which mediate loose interaction between leukocyte and endothelium, such as rolling and tethering. Firm adhesion, usually occurring hours after cytokine-mediated activation, is mediated by the enhanced expressions of VCAM-1 or ICAM-1 that requires transcription and translation of new proteins. ${ }^{28}$ In contrast to this classical pattern of leukocyte/endothelium interactions, we observed a large number of tightly adhered leukocytes in CAV- and L-NMMA-perfused vessels within 10 and 20 min of resumed blood flow, respectively. The adherent leukocytes were tightly attached to the microvessel walls and could not be washed away by reperfusing the vessel with albumin-Ringer perfusate, indicating ICAM-1-mediated adhesion. 
Our results showed a 9.5 fold increase in ICAM-1 immunostaining in intact microvessels following a 30 min CAV perfusion (images in Fig 6). In this study, the anti-ICAM-1 antibody staining was achieved by direct perfusion of intact vessels with the antibody without fixation. Therefore, the observed antibody binding with ICAM-1 should only represent the levels of extracellular domain of ICAM-1 on the surface of endothelial cells. The timing of increased ICAM-1 staining could rule out the possibility of new ICAM-1 synthesis. Studies have demonstrated that mAb1A29 is a specific blocking antibody for ICAM-1 that inhibits ICAM1-mediated leukocyte adhesion. ${ }^{17,} 18$ Our results that perfusing vessels with 1A29 significantly inhibited AP-CAV-induced leukocyte adhesion are consistent with those reports. Then the increased binding affinity of ICAM-1 for this antibody upon AP-CAV perfusion could be the results of increased adhesive binding sites of ICAM-1 for leukocyte adhesion. While the evidence derived from our present study could not rule out the possibility of membrane translocation of ICAM-1 in response to AP-CAV-induced NO reduction, protein analysis from cytoplasmic and membrane fractions in cultured cells reported that TNF-a-induced early phase leukocyte adhesion involves the activation of constitutive ICAM-1 in cell surface. ${ }^{19}$ We also explored the signaling pathways involved in AP-CAV-induced increases in the adhesive activity of ICAM-1. The significant increases in phospho-(Y526)-ICAM-1 staining following AP-CAV perfusion, not AP-CAP-X perfusion, showed a direct correlation between ICAM-1 phosporylation and increased endothelial surface ICAM-1 binding to mAb1A29. The application of a Src kinase inhibitor, PP1, that abolished AP-CAV-induced ICAM-1 phosphorylation and also inhibited AP-CAV-induced leukocyte adhesion supports that Src activation-mediated ICAM-1 phosphorylation resulted in increases in the adhesive activity of ICAM-1. Src activation mediated ICAM-1 
phosphorylation that was independent of protein synthesis has also reported in TNF- $\alpha-$ induced early phase leukocyte adhesion in both cell culture and mouse lung studies ${ }^{19,} 20$. Our results that NOS inhibitor (L-NMMA) showed similar results to those of AP-CAVmediated leukocyte adhesion and that adding exogenous NO donor (SNP), or blocking ICAM-1, prevented AP-CAV-induced leukocyte adhesion, support that reduced basal NOmediated, Src activation-dependent increases in adhesive activity of ICAM-1 is the mechanism for AP-CAV-induced leukocyte adhesion. Most importantly, AP-CAV-induced increases in adhesive activity of ICAM-1 on endothelial cells appears sufficient to mediate firm adhesion of leukocytes within 10-20 min without the need of activating blood cells.

Consistent with our findings, overexpression of caveolin-1 was shown to reduced NO production in vitro, ${ }^{38-40}$ and eNOS-deficient mice show increased levels of leukocyte adhesion. ${ }^{41} \mathrm{~A}$ rapid increase (in $30 \mathrm{~min}$ ) in ICAM-1 expression in thrombin-stimulated umbilical vein endothelial cell was not inhibited by a protein biosynthesis inhibitor, cycloheximide. ${ }^{42}$ However, the mechanisms involved in the anti-inflammatory roles of NO have been attributed to its ability to neutralize superoxide. ${ }^{28,43}$ It has been proposed that eNOS-derived NO does not directly modulate endothelial cell conversion to a pro-adhesive phenotype, but rather interferes with the generation of ROS by NADPH oxidase and prevents ROS-mediated inflammatory response. ${ }^{28,44}$ In contrast to this proposal, the timing and pattern of leukocyte adhesion observed in our studies strongly support a direct association between basal NO and adhesive states of endothelial cells. Although detailed molecular mechanisms remain to be discovered, our present studies indicate that the signaling pathways involved in reduced basal NO-mediated leukocyte adhesion are different from those induced by cytokines. 
Our results also demonstrated that CAV-induced leukocyte adhesion, without fMLP stimulation, does not increase microvessel permeability. The continuous VE-cadherin outlined underneath the AP-CAV-induced adherent leukocytes (Fig 5) provide further evidence that the adhesion process did not cause local changes at endothelial adherent junctions, which is consistent with the permeability measurements. These findings also support that the process of leukocyte recruitments such as adhesion and migration may not necessarily cause vascular leakage. Our results that the increases in microvessel permeability only occurred upon the application of fMLP to vessels with AP-CAV-induced adherent leukocytes, further support that the adhesion process alone can be dissociated with increases in microvessel permeability, and the additional stimulus-induced leukocyte oxidative burst is the direct cause of leukocyte adhesion-mediated increases in microvessel permeability. ${ }^{16,} 45-50$ This could also explain the effective anti-inflammatory actions of systemically applied AP-CAV without reported injury of unaffected organs by several investigators. $11,23,25,29$

\section{Summary}

Our experimental approach allows us to investigate microvessel functions under both basal and stimulated conditions, and in the absence and presence of blood components, thus providing us a unique opportunity to dissect the distinct roles of AP-CAV and NO in the regulation of endothelial function in vivo under different vascular conditions. Our present study on CAV-induced basal NO reduction, in combination with previous studies, demonstrated that basal $\mathrm{NO}$ is essential in maintaining the non-adhesive state of endothelium through regulating of endothelial surface adhesion molecules, whereas the stimulated excessive NO causes increases in microvessel permeability. The reduced basal 
NO-induced increases in adhesive activity of ICAM-1 to leukocytes were mediated by Src activation-dependent ICAM-1 phosphorylation.

\section{GRANT SUPPORT}

This study was supported by National Heart, Lung, and Blood Institute grants HL56237 and HL084338 (He, P.) and American Heart Association Great Rivers Affiliate 12PRE11470010 pre-doctoral fellowship (Xu, S.). 


\section{REFERENCE}

1. He P, Liu B, Curry FE. Effect of nitric oxide synthase inhibitors on endothelial [Ca2+]i and microvessel permeability. Am J Physiol. 1997;272(1 Pt 2):H176-185.

2. Wu HM, Huang $Q$, Yuan $Y$, Granger HJ. VEGF induces NO-dependent hyperpermeability in coronary venules. Am J Physiol. 1996;271(6 Pt 2):H2735-2739.

3. Mayhan WG. Nitric oxide accounts for histamine-induced increases in macromolecular extravasation. Am J Physiol. 1994;266(6 Pt 2):H2369-2373.

4. Kubes $\mathrm{P}$, Granger DN. Nitric oxide modulates microvascular permeability. Am J Physiol. 1992;262(2 Pt 2):H611-615.

5. Kubes P, Suzuki M, Granger DN. Nitric oxide: an endogenous modulator of leukocyte adhesion. Proc Natl Acad Sci U S A. 1991;88(11):4651-4655.

6. Radomski MW, Palmer RM, Moncada S. Endogenous nitric oxide inhibits human platelet adhesion to vascular endothelium. Lancet. 1987;2(8567):1057-1058.

7. Ju H, Zou R, Venema VJ, Venema RC. Direct interaction of endothelial nitric-oxide synthase and caveolin-1 inhibits synthase activity. $J$ Biol Chem. 1997;272(30):18522-18525.

8. Garcia-Cardena G, Martasek P, Masters BS, Skidd PM, Couet J, Li S, Lisanti MP, Sessa WC. Dissecting the interaction between nitric oxide synthase (NOS) and caveolin. Functional significance of the nos caveolin binding domain in vivo. $J$ Biol Chem. 1997;272(41):25437-25440.

9. Zhou X, He P. Endothelial [Ca2+]i and caveolin-1 antagonistically regulate eNOS activity and microvessel permeability in rat venules. Cardiovasc Res. 2010;87(2):340-347.

10. Zhu L, Schwegler-Berry D, Castranova V, He P. Internalization of caveolin-1 scaffolding domain facilitated by Antennapedia homeodomain attenuates PAFinduced increase in microvessel permeability. Am J Physiol Heart Circ Physiol. 2004;286(1):H195-201.

11. Bucci M, Gratton JP, Rudic RD, Acevedo L, Roviezzo F, Cirino G, Sessa WC. In vivo delivery of the caveolin-1 scaffolding domain inhibits nitric oxide synthesis and reduces inflammation. Nat Med. 2000;6(12):1362-1367.

12. Kendall $\mathrm{S}$, Michel $\mathrm{CC}$. The measurement of permeability in single rat venules using the red cell microperfusion technique. Exp Physiol. 1995;80(3):359-372.

13. Curry PE, Huxley $\mathrm{VH}$, Sarelius $\mathrm{IH}$. Techniques in microcirculation: measurement of permeability, pressure and flow. In: Cardiovascular Physiology. Techniques in the Life Sciences. New York: Elsevier; 1983. 
14. Zhou X, He P. Improved measurements of intracellular nitric oxide in intact microvessels using 4,5-diaminofluorescein diacetate. Am J Physiol Heart Circ Physiol. 2011.

15. He $\mathrm{P}$, Zhang $\mathrm{X}$, Curry FE. Ca2+ entry through conductive pathway modulates receptor-mediated increase in microvessel permeability. Am J Physiol. 1996;271(6 Pt 2):H2377-2387.

16. Zhu L, He P. fMLP-stimulated release of reactive oxygen species from adherent leukocytes increases microvessel permeability. Am J Physiol Heart Circ Physiol. 2006;290(1):H365-372.

17. Morisaki H, Suematsu M, Wakabayashi $Y$, Moro-oka S, Fukushima K, Ishimura $Y$, Takeda J. Leukocyte-endothelium interaction in the rat mesenteric microcirculation during halothane or sevoflurane anesthesia. Anesthesiology. 1997;87(3):591-598.

18. Ferrante RJ, Hobson RW, 2nd, Miyasaka M, Granger DN, Duran WN. Inhibition of white blood cell adhesion at reperfusion decreases tissue damage in postischemic striated muscle. J Vasc Surg. 1996;24(2):187-193.

19. Javaid K, Rahman A, Anwar KN, Frey RS, Minshall RD, Malik AB. Tumor necrosis factor-alpha induces early-onset endothelial adhesivity by protein kinase Czetadependent activation of intercellular adhesion molecule-1. Circ Res. 2003;92(10):1089-1097.

20. Liu G, Vogel SM, Gao X, Javaid K, Hu G, Danilov SM, Malik AB, Minshall RD. Src phosphorylation of endothelial cell surface intercellular adhesion molecule-1 mediates neutrophil adhesion and contributes to the mechanism of lung inflammation. Arterioscler Thromb Vasc Biol. 2011;31(6):1342-1350.

21. Zhou X, He P. Temporal and spatial correlation of platelet-activating factor-induced increases in endothelial $[\mathrm{Ca}(2)(+)]$ i, nitric oxide, and gap formation in intact venules. Am J Physiol Heart Circ Physiol. 2011;301(5):H1788-1797.

22. Li S, Couet J, Lisanti MP. Src tyrosine kinases, Galpha subunits, and H-Ras share a common membrane-anchored scaffolding protein, caveolin. Caveolin binding negatively regulates the auto-activation of Src tyrosine kinases. J Biol Chem. 1996;271(46):29182-29190.

23. Bucci M, Roviezzo F, Posadas I, Yu J, Parente L, Sessa WC, Ignarro LJ, Cirino G. Endothelial nitric oxide synthase activation is critical for vascular leakage during acute inflammation in vivo. Proc Natl Acad Sci U S A. 2005;102(3):904-908.

24. Zhu L, He P. Platelet-activating factor increases endothelial $[\mathrm{Ca} 2+] \mathrm{i}$ and $\mathrm{NO}$ production in individually perfused intact microvessels. Am J Physiol Heart Circ Physiol. 2005;288(6):H2869-2877. 
25. Gratton JP, Lin MI, Yu J, Weiss ED, Jiang ZL, Fairchild TA, Iwakiri Y, Groszmann R, Claffey KP, Cheng YC, Sessa WC. Selective inhibition of tumor microvascular permeability by cavtratin blocks tumor progression in mice. Cancer Cell. 2003;4(1):31-39.

26. Bernatchez PN, Bauer PM, Yu J, Prendergast JS, He P, Sessa WC. Dissecting the molecular control of endothelial NO synthase by caveolin-1 using cell-permeable peptides. Proc Natl Acad Sci U S A. 2005;102(3):761-766.

27. Yao SK, Ober JC, Krishnaswami A, Ferguson JJ, Anderson HV, Golino P, Buja LM, Willerson JT. Endogenous nitric oxide protects against platelet aggregation and cyclic flow variations in stenosed and endothelium-injured arteries. Circulation. 1992;86(4):1302-1309.

28. Kvietys PR, Granger DN. Role of reactive oxygen and nitrogen species in the vascular responses to inflammation. Free Radic Biol Med. 2012;52(3):556-592.

29. Cirino G, Fiorucci S, Sessa WC. Endothelial nitric oxide synthase: the Cinderella of inflammation? Trends Pharmacol Sci. 2003;24(2):91-95.

30. Kuhlencordt PJ, Rosel E, Gerszten RE, Morales-Ruiz M, Dombkowski D, Atkinson WJ, Han F, Preffer F, Rosenzweig A, Sessa WC, Gimbrone MA, Jr., Ertl G, Huang $\mathrm{PL}$. Role of endothelial nitric oxide synthase in endothelial activation: insights from eNOS knockout endothelial cells. Am J Physiol Cell Physiol. 2004;286(5):C11951202.

31. Duran WN, Breslin JW, Sanchez FA. The NO cascade, eNOS location, and microvascular permeability. Cardiovasc Res.87(2):254-261.

32. Kurose I, Wolf R, Grisham MB, Granger DN. Effects of an endogenous inhibitor of nitric oxide synthesis on postcapillary venules. Am J Physiol. 1995;268(6 Pt 2):H2224-2231.

33. Kurose I, Kubes P, Wolf R, Anderson DC, Paulson J, Miyasaka M, Granger DN. Inhibition of nitric oxide production. Mechanisms of vascular albumin leakage. Circ Res. 1993;73(1):164-171.

34. Rumbaut RE, Wang J, Huxley VH. Differential effects of L-NAME on rat venular hydraulic conductivity. Am J Physiol Heart Circ Physiol. 2000;279(4):H2017-2023.

35. Mayhan WG. Role of nitric oxide in modulating permeability of hamster cheek pouch in response to adenosine 5'-diphosphate and bradykinin. Inflammation. 1992;16(4):295-305.

36. Pober JS, Sessa WC. Evolving functions of endothelial cells in inflammation. Nat Rev Immunol. 2007;7(10):803-815. 
37. Ley K, Laudanna C, Cybulsky MI, Nourshargh S. Getting to the site of inflammation: the leukocyte adhesion cascade updated. Nat Rev Immunol. 2007;7(9):678-689.

38. Fernandez-Hernando C, Yu J, Davalos A, Prendergast J, Sessa WC. Endothelialspecific overexpression of caveolin-1 accelerates atherosclerosis in apolipoprotein E-deficient mice. Am J Pathol. 2010;177(2):998-1003.

39. Fernandez-Hernando C, Yu J, Suarez Y, Rahner C, Davalos A, Lasuncion MA, Sessa WC. Genetic evidence supporting a critical role of endothelial caveolin-1 during the progression of atherosclerosis. Cell Metab. 2009;10(1):48-54.

40. Frank PG, Lee H, Park DS, Tandon NN, Scherer PE, Lisanti MP. Genetic ablation of caveolin-1 confers protection against atherosclerosis. Arterioscler Thromb Vasc Biol. 2004;24(1):98-105.

41. Lefer DJ, Jones SP, Girod WG, Baines A, Grisham MB, Cockrell AS, Huang PL, Scalia R. Leukocyte-endothelial cell interactions in nitric oxide synthase-deficient mice. Am J Physiol. 1999;276(6 Pt 2):H1943-1950.

42. Sugama $Y$, Tiruppathi $C$, offakidevi $K$, Andersen TT, Fenton JW, 2nd, Malik AB. Thrombin-induced expression of endothelial P-selectin and intercellular adhesion molecule-1: a mechanism for stabilizing neutrophil adhesion. J Cell Biol. 1992;119(4):935-944.

43. Kurose I, Wolf R, Grisham MB, Aw TY, Specian RD, Granger DN. Microvascular responses to inhibition of nitric oxide production. Role of active oxidants. Circ Res. 1995;76(1):30-39.

44. Niu XF, Smith CW, Kubes P. Intracellular oxidative stress induced by nitric oxide synthesis inhibition increases endothelial cell adhesion to neutrophils. Circ Res. 1994;74(6):1133-1140.

45. Zeng $\mathrm{M}$, Zhang $\mathrm{H}$, Lowell $\mathrm{C}$, He $\mathrm{P}$. Tumor necrosis factor-alpha-induced leukocyte adhesion and microvessel permeability. Am J Physiol Heart Circ Physiol. 2002;283(6):H2420-2430.

46. Zhu L, Castranova V, He P. fMLP-stimulated neutrophils increase endothelial [Ca2+]i and microvessel permeability in the absence of adhesion: role of reactive oxygen species. Am J Physiol Heart Circ Physiol. 2005;288(3):H1331-1338.

47. Tinsley JH, Ustinova EE, Xu W, Yuan SY. Src-dependent, neutrophil-mediated vascular hyperpermeability and beta-catenin modification. Am J Physiol Cell Physiol. 2002;283(6):C1745-1751.

48. Jakus Z, Berton G, Ligeti E, Lowell CA, Mocsai A. Responses of neutrophils to antiintegrin antibodies depends on costimulation through low affinity Fc gamma Rs: full activation requires both integrin and nonintegrin signals. $J$ Immunol. 2004;173(3):2068-2077. 
49. Harris NR, Benoit JN, Granger DN. Capillary filtration during acute inflammation: role of adherent neutrophils. Am J Physiol. 1993;265(5 Pt 2):H1623-1628.

50. He P. Leucocyte/endothelium interactions and microvessel permeability: coupled or uncoupled? Cardiovasc Res. 2010;87(2):281-290. 
FIGURES AND FIGURE LEGENDS

Figure 1
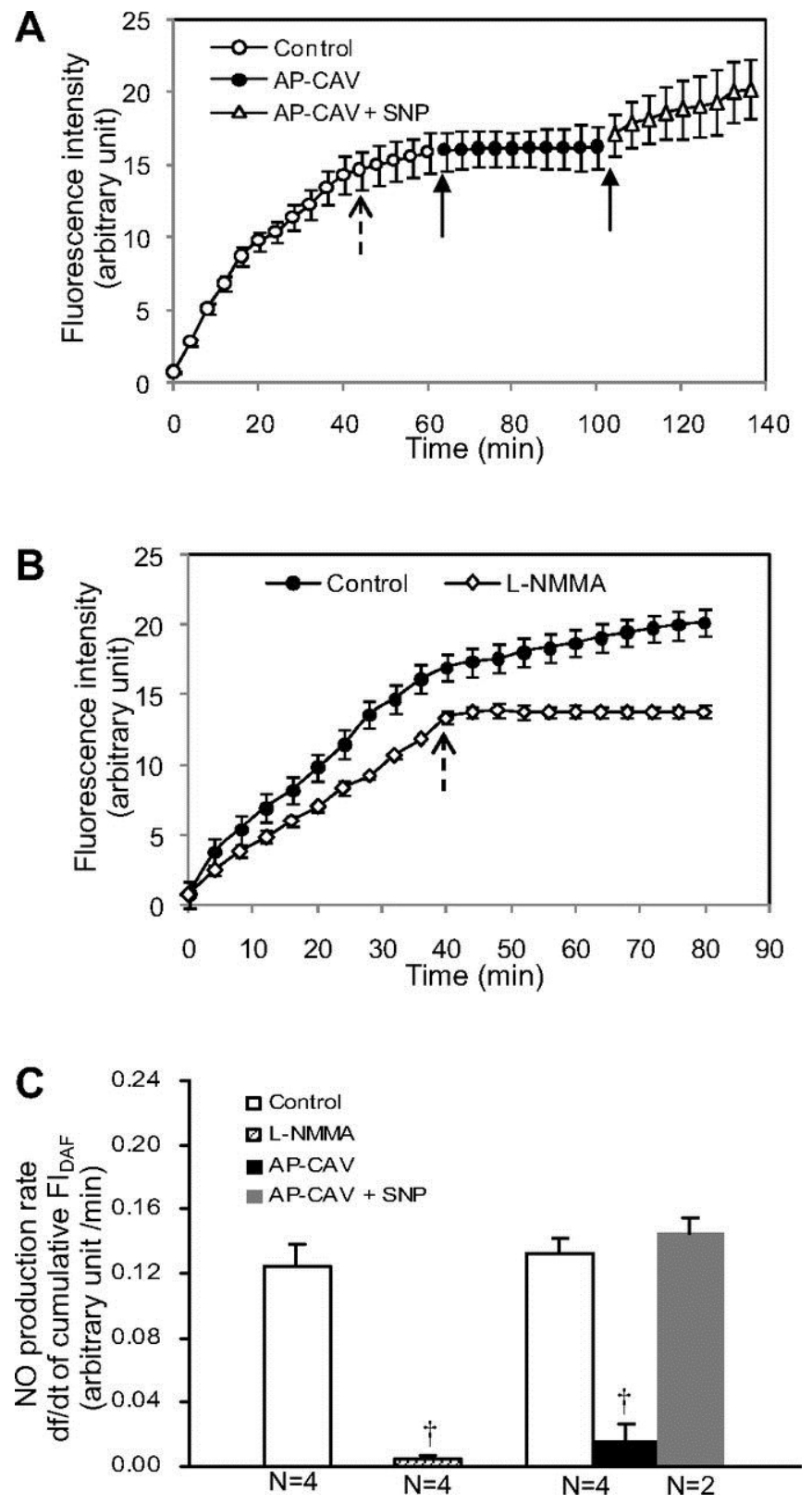
Figure 1. The application of Antennapedia homeodomain (AP)-caveolin-1 (CAV) inhibits basal nitric oxide (NO). A: the time-dependent changes in cumulative DAF-2 fluorescence intensity (FIDAF) from one representative experiment before $(\circ)$ and after $(\bullet)$ treatment with $10 \mu \mathrm{M}$ AP-CAV, followed by $10 \mu \mathrm{M}$ SNP $(\Delta)$ perfusion for $40 \mathrm{~min}$. B: timedependent changes in cumulative FIDAF from two representative experiments in the absence $(\bullet)$ or presence of $(\circ)$ of $500 \mu \mathrm{M}$ I-NMMA. C: summary of NO production rate derived from cumulative FIDAF in each group of vessels. The dashed arrow indicates the time when DAF-2 fluorescence reached steady state. The arrow indicates the time when testing solution was added. †Significant decreases from control $(P<0.05)$. 
Figure 2
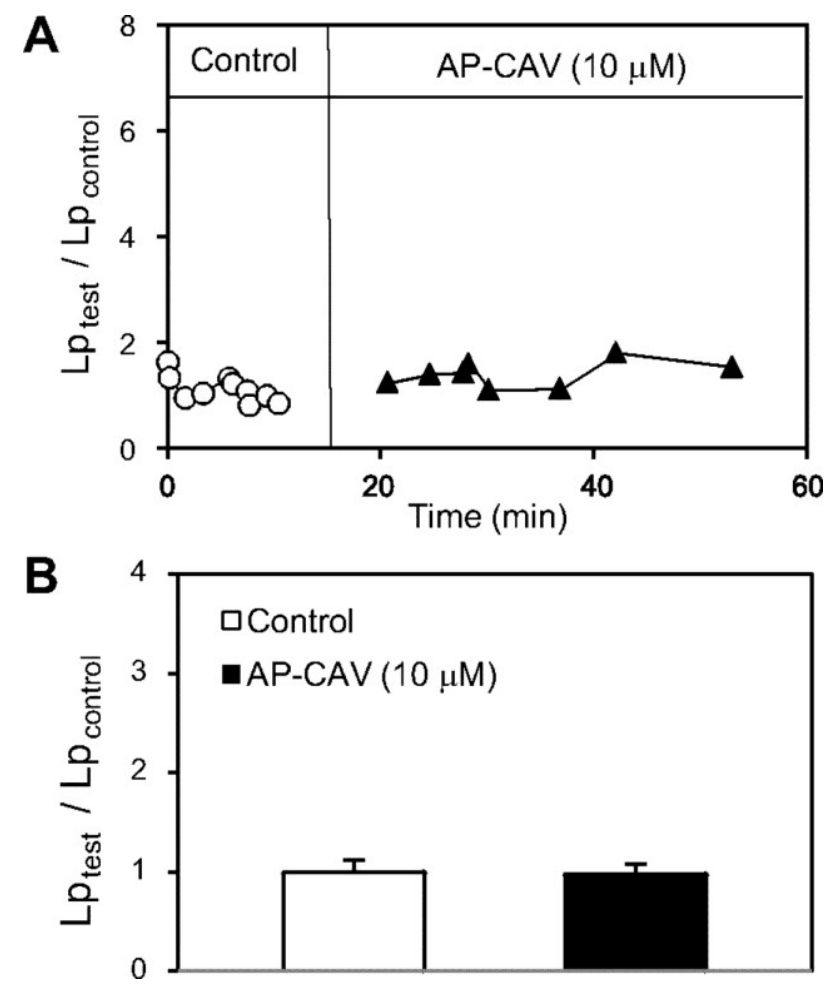

Figure 2. Perfusion of AP-CAV for $30 \mathrm{~min}$ has no effect on basal hydraulic conductivity (Lp). A: a representative experiment shows the time course of $L p$ changes when the vessel was perfused with AP-CAV $(10 \mu \mathrm{M})$. B: summary results of four experiments. 
Figure 3
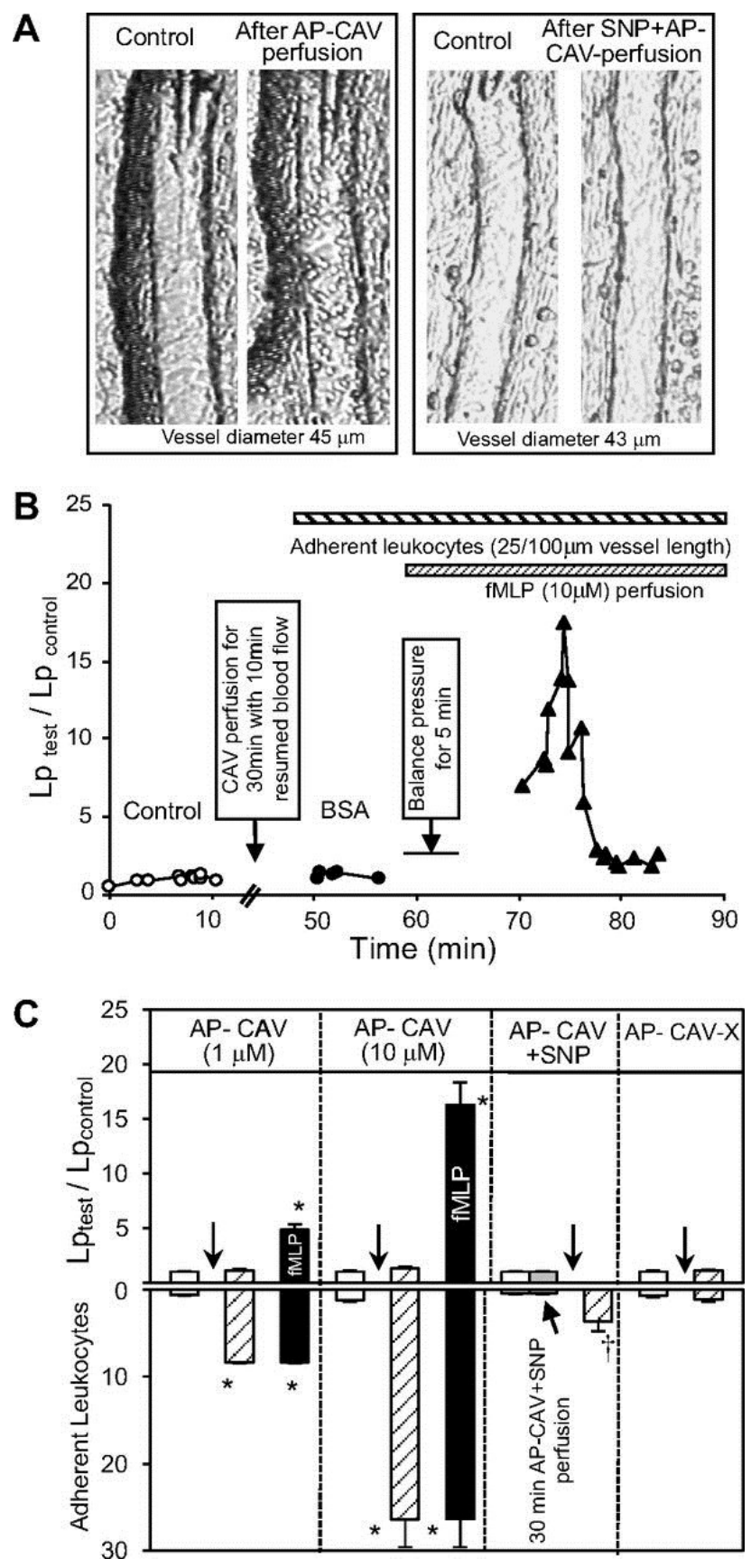
Figure 3. Reduction of basal NO by AP-CAV induced significant leukocyte adhesion without increasing $L p$ in the absence of a secondary stimulation. A: paired video images from two rat venules. The two images on the left show the same vessel under control conditions and after AP-CAV $(10 \mu \mathrm{M})$-induced leukocyte adhesion. The two images on the right show that the application of sodium nitroprusside (SNP) abolished AP-CAVinduced leukocyte adhesion. B: the time course of $L p$ changes from a representative experiment showing that AP-CAV $(10 \mu \mathrm{M})$-induced leukocyte adhesion $(25 / 100 \mu \mathrm{m}$ of vessel length) did not increase Lp, unless formyl-Met-Leu-Phe-OH (fMLP) (10 $\mu \mathrm{M})$ was added to the perfusate. C: summary graph showing the correlation between the number of adherent leukocytes (per $100 \mu \mathrm{m}$ vessel length) and the changes in Lp in four group of studies. Perfusion vessels with AP-CAV at $1 \mu M(n=3)$ and $10 \mu M(n=5)$ show AP-CAV dose-dependent increases in leukocyte adhesion and fMLP-induced increases in Lp. The application of SNP attenuated AP-CAV-induced leukocyte adhesion $(n=4)$, and replacing AP-CAV with scrambled AP-CAV (AP-CAV-X) showed no effect on leukocyte adhesion (n $=3)$. The blank bars represent the control values. The arrows indicate the procedures of $30 \mathrm{~min}$ of AP-CAV or AP-CAV-X perfusion followed by $10 \mathrm{~min}$ of resumed blood flow. The dashed line bars represent values measured after resumed blood flow in AP-CAV or APCAV-X perfused vessels. *Significant increases from the control values $(P<0.05)$. †Significant decreases from AP-CAV group $(P<0.05)$. 
Figure 4
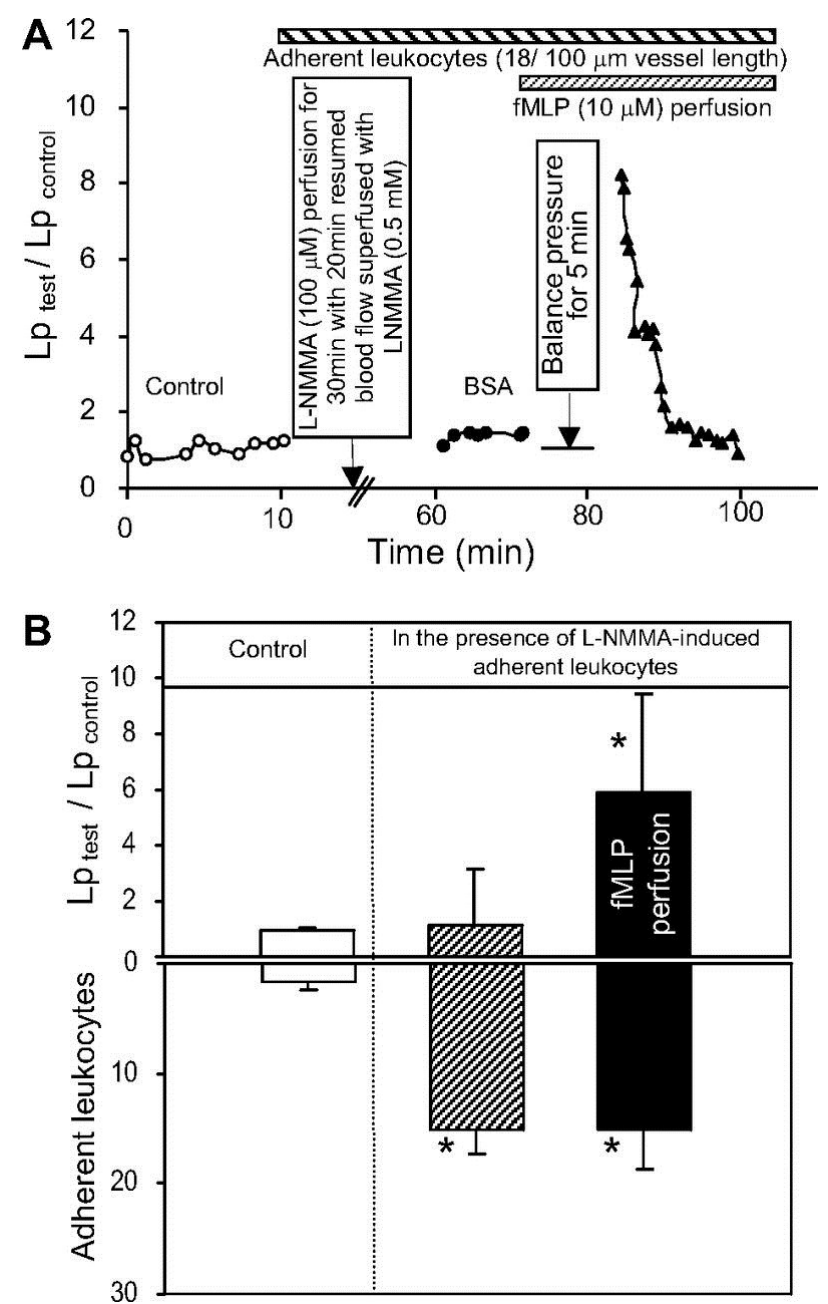

Figure 4. L-NMMA, a NO synthase (NOS) inhibitor, showed similar effects on leukocyte adhesion and microvessel permeability to those of CAV. A: time course of Lp changes from a representative experiment. B: summary of the number of adherent leukocytes (per 100- $\mu \mathrm{m}$ vessel length) and the corresponding changes in microvessel Lp $(n=3) .{ }^{*}$ Significant increases from control $(P<0.05)$. 
Figure 5

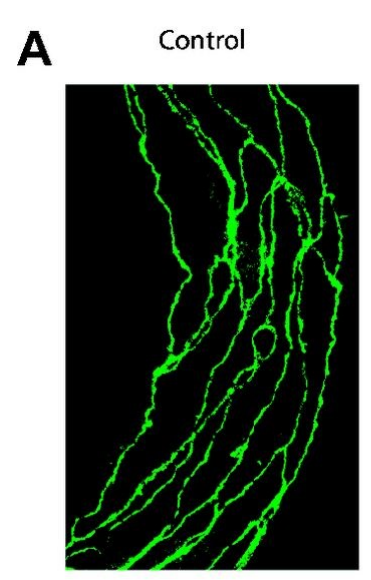

VE-cadherin

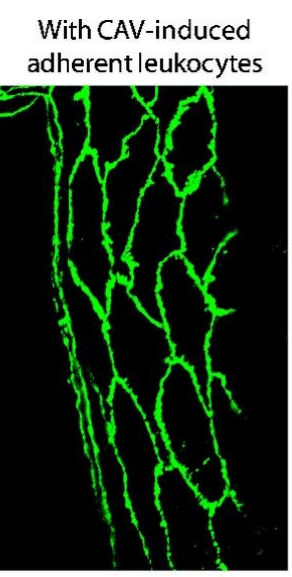

VE-cadherin
With CAV-induced

adherent leukocytes

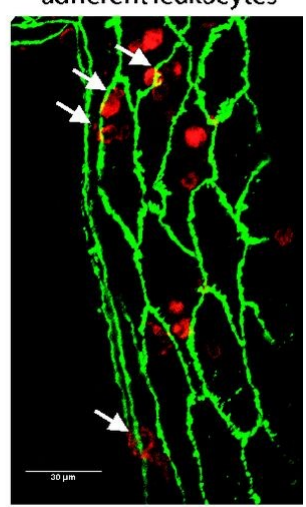

VE-cadherin and leukocytes

B
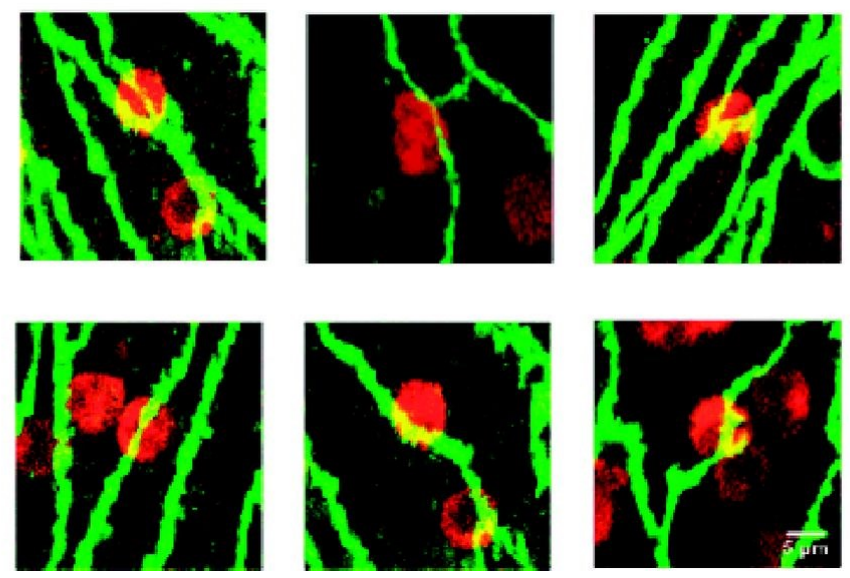

Figure 5. Confocal images of the co-staining of VE-cadherin and adherent leukocytes

illustrating that CAV-induced leukocyte adhesion did not change VE-cadherin distribution. A: VE-cadherin staining under control conditions and after CAV-induced leukocyte adhesion. The third image shows the double staining of VE-cadherin and adherent leukocytes (indicated by arrows). B: magnified images from three different vessels showing no changes in VE-cadherin at the adhesion sites. 
Figure 6.

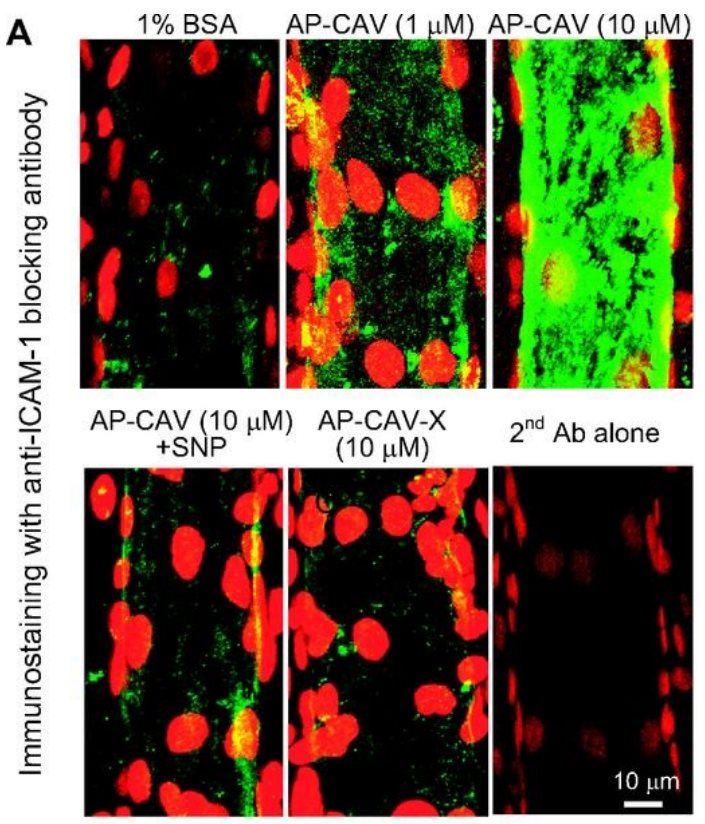

B
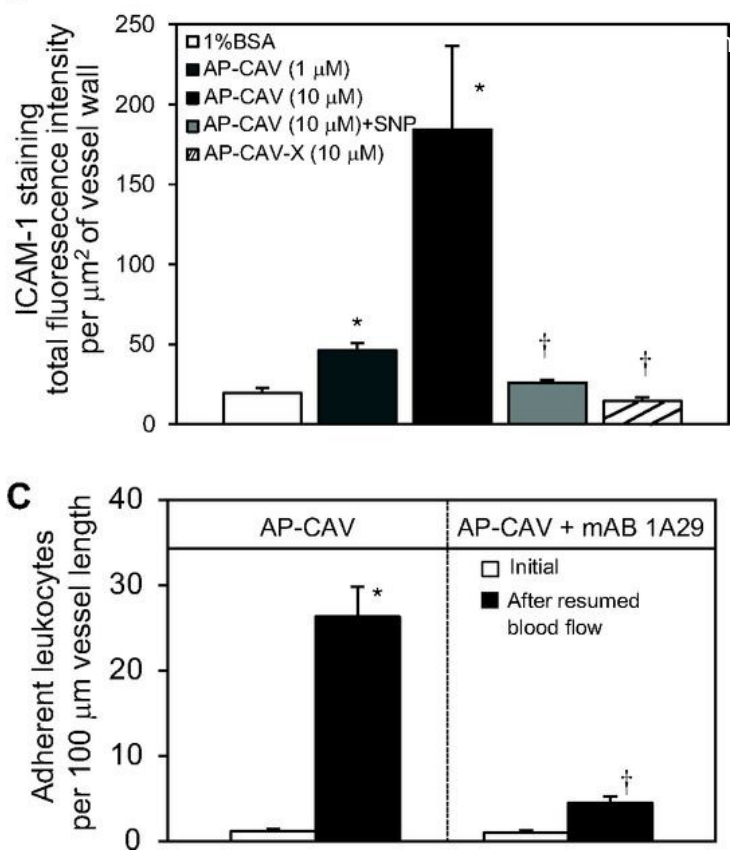
Figure 6. AP-CAV-induced increase in ICAM-1 adhesive capacity causes leukocyte adhesion. $A$ : representative confocal images of anti-ICAM-1 blocking antibody mAb1A29 and vascular cell nuclei co-staining from five groups of studies and the secondary antibody control (ICAM-1 is shown in green, and nuclei are red). B: quantification of total fluorescence intensity $(\mathrm{FI})$ of ICAM-1 per unit area of vessel wall under control conditions $(n=4)$, after AP-CAV $(1 \mu \mathrm{M}, n=3$; and $10 \mu \mathrm{M}, n=4)$ perfusion in the absence and presence of SNP $(n=3)$, and after AP-CAV-X perfusion $(n=3)$. C: perfusion of vessels with mAb1A29 significantly attenuated AP-CAV (10 $\mu \mathrm{M})$-induced leukocyte adhesion ( $n=5$ per group). ${ }^{*}$ Significant increase from control $(P<0.05)$. $†$ Significant decrease from AP-CAV group $(P$ $<0.05)$. 
Figure 7.
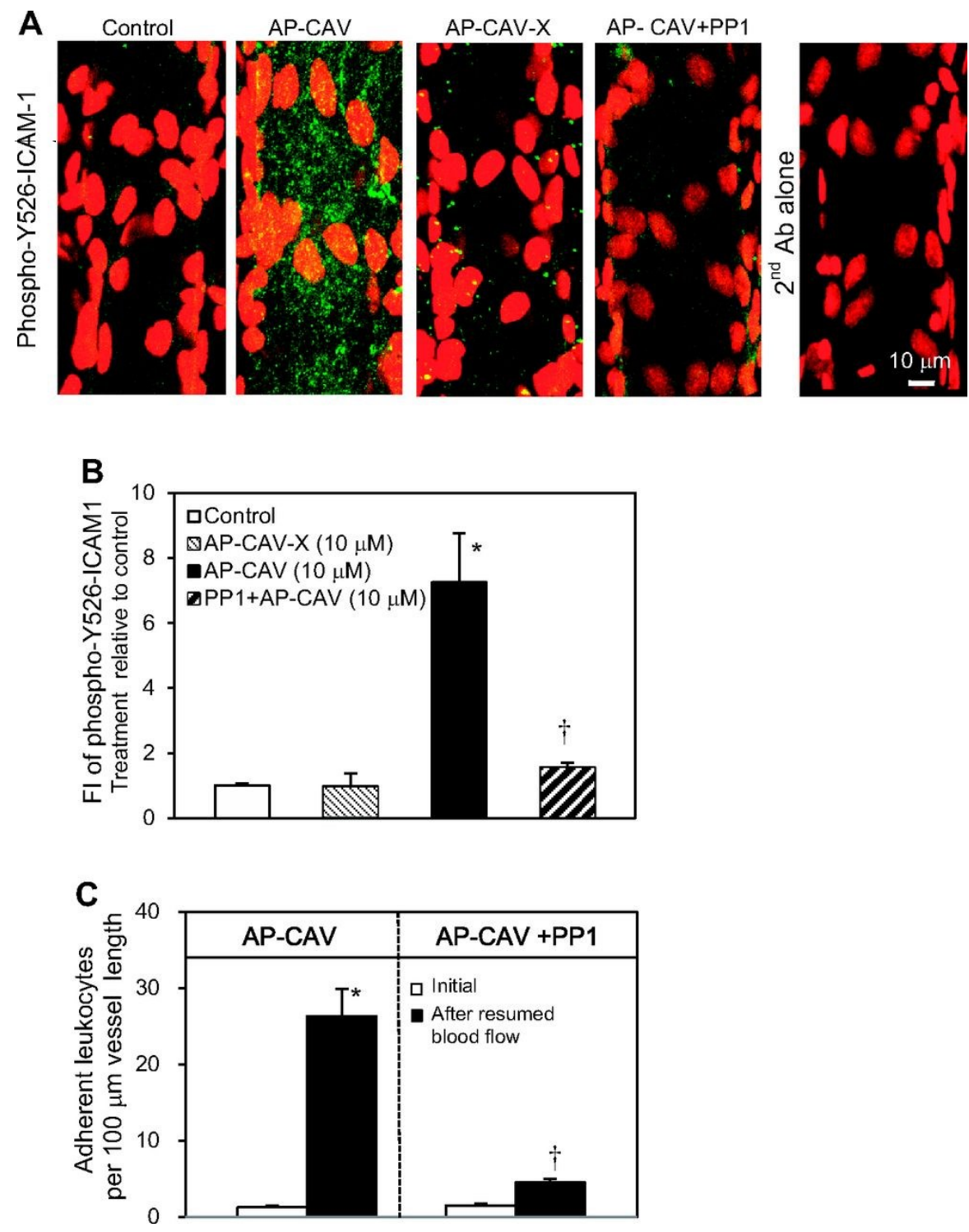
Figure 7. AP-CAV-induced leukocyte adhesion involves Src activation-mediated ICAM-1 phosphorylation at tyrosine 526 (Y526). A: representative confocal images demonstrate increases in ICAM-1 phosphorylation at Y526 following AP-CAV perfusion relative to that of the control. Such increased ICAM-1 phosphorylation was blocked by the application of a Src kinase inhibitor, PP1, and was absent in vessels perfused with APCAV-X or secondary antibody alone. Phosphorylated ICAM-1 at Y526 is shown in green, and nuclei are red. B: summary of changes in FI of phospho-Y526-ICAM-1 relative to control in each experimental group ( $n=3$ per group). $C$ : perfusion of vessels with PP1 that prevented ICAM-1 phosphorylation also significantly attenuated AP-CAV induced leukocyte adhesion $(n=3)$. *Significant increase from control $(P<0.05)$. †Significant decrease from AP-CAV group $(P<0.05)$. 


\title{
CHAPTER 3
}

SHEAR STRESS-INDUCED ENDOTHELIAL CELL SIGNALING AND BARRIER

FUNCTION IN INTACT MICROVESSELS: INTEGRATIVE ROLES OF FLUID-

\section{GENERATED WALL SHEAR STRESS AND SHEAR-INDUCED RELEASES OF ATP \\ FROM RED BLOOD CELLS}

\author{
Sulei Xu, Stanley D. Yokota, Pingnian He
}

Department of Physiology and Pharmacology, School of medicine, West Virginia University, Morgantown, WV 26506

Short Title: Shear stress and microvessel permeability

First author's surname: Xu

Address for proofs:

Dr. Pingnian $\mathrm{He}$

Department of Physiology and Pharmacology

School of Medicine

West Virginia University

Morgantown, WV 26506-9229

Tel: (304) 293-1515

Fax: (304) 293-3850

E-Mail: phe@hsc.wvu.edu

Subject Code: [95] Endothelium/vascular type/nitric oxide, [96] Mechanism of atherosclerosis/growth factors 


\begin{abstract}
Rationale Acute changes of shear stress (SS) have been recognized to play important roles in vascular dysfunctions. However, detailed mechanisms involved in SS-induced endothelial cell (EC) signaling and vascular barrier function in vivo have not been well understood, especially in the presence of red blood cells (RBCs).
\end{abstract}

Objective This study investigates the effects of acute changes of SS on EC $\left[\mathrm{Ca}^{2+}\right]$ i, eNOS activation, nitric oxide (NO) production, and vascular barrier function in individually perfused intact rat mesenteric venules.

Methods and Results SS was quantified by measuring maximal flow velocity and fluid viscosity using a high-speed camera and a cone-plate viscometer. $\mathrm{EC}\left[\mathrm{Ca}^{2+}\right]_{i}$ and $\mathrm{NO}$ were measured in Fura-2 AM and DAF-2 DA loaded venules. EC eNOS activation was evaluated by immunofluorescence staining with confocal imaging. ATP released from RBCs was measured by bioluminescence assay. In response to changes of SS, transient SSdependent increases in EC $\left[\mathrm{Ca}^{2+}\right]$ i occurred only in vessels perfused with whole blood or perfusate containing RBCs, which were correlated with EC gap formation illustrated by fluorescent microsphere accumulation. Whereas, both plasma and whole blood perfusion induced SS-dependent NO production and eNOS-Ser ${ }^{1177}$ phosphorylation. Carbenoxolone, a Pannexin 1 inhibitor, abolished SS-dependent ATP release from RBCs and also prevented SS-induced increases in EC $\left[\mathrm{Ca}^{2+}\right]$ and EC gap formation. 
Conclusions These results indicate that SS-induced ATP release from RBCs causes an increase in EC $\left[\mathrm{Ca}^{2+}\right]$ and vascular permeability, and that SS-induced NO production can be independent of increased EC $\left[\mathrm{Ca}^{2+}\right]$ in intact venules.

Keywords: shear stress; microvessel; permeability

\section{Non-standard Abbreviations and Acronyms}

$\mathrm{SS}=$ shear stress

$\mathrm{EC}=$ endothelial cell

$\mathrm{NO}=$ nitric oxide

eNOS $=$ endothelial nitric oxide synthase

$\mathrm{RBC}=$ red blood cell 


\section{INTRODUCTION}

In the circulatory system, sustained fluid shear stress (SS) has been implicated to play important roles in maintaining vascular wall endothelium integrity, and changes of SS or disrupted flow patterns contribute to the pathogenesis of many disease-associated vascular dysfunction. ${ }^{1}$ Although the effects of SS on endothelial cell (EC) functions have been extensively investigated in both cell culture and in intact microvessels, the results are still controversial and the detailed mechanisms remain not well understood. Most of the SSrelated studies were conducted in cultured endothelial monolayers, which have provided important means to elucidate the relationship between SS and endothelial function. ${ }^{2-7}$ However, it has also been recognized that the cultured ECs grown under static conditions usually have 10-100 times higher baseline permeability compared to intact microvessels, and have been considered as pro-inflammatory phenotype, ${ }^{8}$ therefore, their responses to mechanical forces may not fully replicate in vivo conditions. Additionally, under in vivo conditions, the fluid in the vascular system is not a cell-free fluid. It contains blood cells that also subject to SS under different flow conditions. Studies on red blood cells (RBCs) have indicated that SS plays important roles in triggering RBC to release bioactive agents, such as ATP, that regulate many important vascular functions. ${ }^{9-13}$ However, previous studies of the effects of SS on vascular endothelium, either in cultured endothelial monolayers ${ }^{2-7}$ or in individually perfused microvessels, ${ }^{14-16}$ were mainly conducted in the absence of blood cell components, whereas the studies of the effects of SS on blood cells, such as RBCs, usually do not involve vascular endothelium. ${ }^{9-13}$

Considering current contradictory results relating to the effects of SS on ECs, such as the SS-induced changes of EC $\left[\mathrm{Ca}^{2+}\right]$, the $\mathrm{Ca}^{2+}$ dependence of SS-induced NO production, 
and the effect of changing of SS on vascular permeability, our study aims to provide a better understanding of these issues by focusing on direct comparisons of the effects of SS generated by cell-free fluid (i.e. plasma and dextran solutions) and whole blood on EC $\left[\mathrm{Ca}^{2+}\right] \mathrm{i}, \mathrm{NO}$ production, and EC barrier integrity using individually perfused intact venules in rat mesentery. ECs respond differently to constant versus transient changes of SS. ${ }^{1}$ Our studies were focused on the effects of transient increases in SS. The changes of SS were generated by changing flow velocity and perfusion pipet diameter and mimic some of the geometric or pathological changes that occur in the vasculature in vivo. We first quantified the wall SS by measuring the maximal RBC velocity and fluid viscosity using a high-speed camera and a cone-plate viscometer. $\mathrm{EC}\left[\mathrm{Ca}^{2+}\right]_{i}$ and nitric oxide (NO) were measured in Fura-2 AM and DAF-2 DA loaded venules when the SS was generated by different perfusates. EC eNOS activation was evaluated by immunofluorescence staining with confocal imaging. SS-induced ATP released from RBCs is measured by bioluminescence assay. Changes of microvessel permeability upon changing SS was evaluated by the endothelial gap formations using fluorescence microspheres. The involvement of pannexin 1 channel on RBCs in SS-induced ATP release and its relationship with endothelial $\mathrm{Ca}^{2+}$ and microvessel permeability were also investigated. 


\section{METHODS}

\section{Animal preparation}

Experiments were carried out on venular microvessels in rat mesenteries. Female SpragueDawley rats (2-3-mo old; 220-250 g body wt; Hilltop Lab Animals, Inc.; Scottdale, PA) were used for all of the experiments. Sodium pentobarbital was used for anesthesia and given subcutaneously at an initial dosage of $65 \mathrm{mg} / \mathrm{kg}$ body weight with an additional 3-mg dose given as needed. The trachea was intubated, and a midline surgical incision $(1.5-2 \mathrm{~cm})$ was made in the abdominal wall. A loop of the ileum was gently extended from the abdominal cavity and the mesentery spread over a glass cover slip attached to an animal tray. The upper surface of the mesentery was continuously superfused with mammalian Ringer's solution, maintained at $37^{\circ} \mathrm{C}$. All experiments were carried out on venular microvessels with diameters ranging between 40 to $45 \mu \mathrm{m}$. Each experiment was performed on a single microvessel with one experiment per animal. The investigation conforms to the Guide for the Care and Use of Laboratory Animals (NRC Eighth edition 2010). All procedures were approved by the West Virginia University Animal Care and Use Committee.

\section{Quantification of shear stress in individually perfused venules.}

Wall SS, the tangential force exerted per unit area of fluid-endothelium surface, is determined by shear rate and viscosity. Wall Shear rate $(\mathrm{V})$, determined by mean fluid velocity ( $\left.\mathrm{V}_{\text {mean }}\right)$ and vessel diameter $(\mathrm{D})$, is calculated by the equation $\mathrm{y}=8 \mathrm{~V}_{\text {mean }} / \mathrm{D} \cdot{ }^{17} \mathrm{~A}$ Photron-FASTCAM high-speed camera was used to record images at 500 frames/sec for flow velocity measurements. ImageJ analysis software with the MTrackJ plugin was used to quantify the center line red blood cell velocity ( $V \max$ ), the maximum red blood cell velocity in laminar flow 
parabolic profile. The mean fluid velocity (Vmean) is calculated as Vmax / 1.6. ${ }^{17}$ Perfusate viscosity was measured using a Wells-Brookfield cone/plate digital viscometer (LVTDCP). In the designed experiments, a single venular microvessel was cannulated with a glass micropipette and perfused with albumin-Ringer solution (control) or other perfusate solutions. Hydrostatic pressure, controlled by a water manometer, was applied through the micropipette to the microvessel lumen to control the flow rate. Wall shear stress was modulated by changing shear rate through changing perfusion pressure or the composition of the perfusate.

\section{Measurements of endothelial $\left[\mathrm{Ca}^{2+}\right]_{\mathrm{i}}$}

Endothelial $\left[\mathrm{Ca}^{2+}\right]$ i was measured in individually perfused microvessels using the fluorescent $\mathrm{Ca}^{2+}$ indicator fura 2-AM. Experiments were performed on a Nikon Diaphot 300 inverted microscope equipped with a Nikon photometry system including photometer head and finder (P101), computer-controlled shutter, and filter changer (Lambda 10-2; Sutter Instrument; Novato, CA). A rectangular variable diaphragm located in the photometer finder determined the size of the measuring window through which the fluorescence intensity $(\mathrm{FI})$ was collected. A segment of fura 2-AM-loaded vessel, at least $100 \mu \mathrm{m}$ away from the cannulation pipet site assuring laminar flow, was then positioned within the field of view of the measuring window. The size of the window covered about 50 endothelial cells forming the vessel wall. A Nikon Fluor lens (x20, numerical aperture, 0.75$)$ was used to collect FI values. The excitation wavelengths for fura 2-AM were selected by two narrow-band interference filters (340 \pm 5 and $380 \pm 5 \mathrm{~nm}$; Oriel), and the emission was separated with a dichroic mirror (DM400) and a wide-band interference filter $(500 \pm 35 \mathrm{~nm}$; Oriel). The exposure time is $0.25 \mathrm{~s}$. The ratios 
of the two $\mathrm{FI}$ values were converted to $\mathrm{Ca}^{2+}$ concentrations using an in vitro calibration curve. A detailed description has been previously reported. ${ }^{18}$

\section{Measurements of endothelial nitric oxide}

Endothelial NO production was measured in DAF-2 DA loaded vessels using fluorescence imaging. Experiments were performed on a Nikon Diaphot 300 microscope equipped with a 12-bit digital CCD camera (ORCA; Hamamatsu) and a computer controlled shutter (Lambda 10-2; Sutter Instrument; Novato, CA). A 75-W xenon lamp was used as the light source. The excitation wavelength for DAF-2 was selected by an interference filter (480/40 $\mathrm{nm})$, and emission was separated by a dichroic mirror ( $505 \mathrm{~nm})$ and a band-pass barrier $(535 / 50 \mathrm{nM})$. All the images were acquired and analyzed using Metafluor software (Universal Imaging, West Chester, PA). To minimize the photo-bleaching, a neutral density filter (0.5 ND) was positioned in front of the interference filter and the exposure time was minimized to $0.12 \mathrm{sec}$ at 1 min intervals. During the experiments, each vessel was first perfused with albumin-Ringer solution containing DAF-2 DA (5 $\mu \mathrm{M})$. Image collection was started after 40 minutes of DAF-2 DA perfusion. All images were collected from a group of endothelial cells located in the same focal plane of the vessel wall using a Nikon Fluor lens (x20, numerical aperture, 0.75). Quantitative analysis was conducted at the individual endothelial cell level using manually selected regions of interest (ROls) along the vessel wall. Each ROI covers the area of one individual cell as indicated by the fluorescence outline. The tissue autofluorescence was subtracted from all of the measured Fls. Shear stress-induced changes in fluorescence intensity $(\mathrm{Fl})$ were expressed as $(\mathrm{Fl} / \mathrm{Fl} 0) \times 100$, 
where $\mathrm{Fl}_{\mathrm{o}}$ is control $\mathrm{Fl}$ level. The NO production rate was calculated as $\mathrm{dFI} / \mathrm{dt}$. Details have been described previously. ${ }^{19}$

\section{RBC preparation and ATP measurement}

Blood was draw from the carotid artery with heparin $(20 \mathrm{unit} / \mathrm{ml})$ and was used immediately. The blood was diluted to approximately $1 \%$ Hct in mammalian Ringer's solution. The blood suspension was then centrifuged at $500 \times \mathrm{g}$ for $5 \mathrm{~min}$ and the buffy coat removed. This procedure was repeated another two times to obtain washed RBCs. Blood samples were kept at room temperature throughout experiments. Packed RBCs were resuspended in $1 \%$ albumin-Ringer's solution. Immediately following exposure to shear stress in the viscometer, $\mathrm{RBC}$ samples were removed and were mixed 50/50 with the luciferin/luciferase assay mix (Sigma). The ATP level in each sample was measured for $20 \mathrm{sec}$ using a luminometer (AutoLumatPlus LB953, Berthold). The relative light unit (I) is proportional to the concentration of extracellular ATP. A zero-shear control was taken for each prepared solution (lo). The relative ATP release is reported as $\left(\mathrm{I}-\mathrm{I}_{0}\right) / \mathrm{I}_{0}$.

\section{Immunofluorescence staining and confocal imaging}

The mesentery bearing each perfused vessel was fixed with paraformaldehyde, followed by permeabilization with $0.1 \%$ Triton $\mathrm{X}-100$ before exposure to the anti-phosphorylated eNOS at Ser ${ }^{1177}$ (Abcam) or Thr ${ }^{495}$ (Cell Signaling Technology) antibody. The tissue was then incubated with Alexa 488-conjugated secondary antibody (Invitrogen) at room temperature for $2 \mathrm{~h}$. Confocal images were obtained using a Leica objective x63 (HCX PL APO, NA 1.2) with $\times 1.5$ electronic zoom, and the vertical step was $0.3 \mu \mathrm{m}$. The mean FI of individual endothelial cells was quantified by selecting three one square micron ROls that 
within each cell and averaging the Z-axis stack of each ROI, using Leica confocal software. The mean of the FI averaged from three stacks of ROIs estimated the individual endothelial cell mean FI. The mean FI for each vessel segment was estimated by averaging the mean endothelial cell Fls for three endothelial cells, and represented site specific eNOS phosphorylation.

\section{Visualization of endothelial gap formation in intact microvessels}

We used fluorescent microspheres (FMs) as the marker to identify endothelial gap formation. Briefly, each individual microvessel was perfused with a perfusate containing red FMs $\left(100 \mathrm{~nm}, 3.6 \times 10^{11} / \mathrm{ml}\right)$ for $10 \mathrm{~min}$ with and without changes in SS. Confocal images were collected following 10 min albumin-Ringer perfusion to remove the free FMs from the vessel lumen. A stack of confocal images was obtained from each vessel at successive X$Y$ focal planes with vertical depth of $0.5 \mu \mathrm{m}$ using Leica $\times 25$ (NA 0.95) objective. The accumulation of FMs at junctions between ECs was analyzed in images from lower half of the complete Z-axis image stack of the vessel. The total FI of FM (area $x$ depth $x$ mean intensity/pixel) for each vessel segment was quantified as total intensity/surface area of the vessel wall under control or shear conditions. Details have been described previously. ${ }^{20}$

\section{Measurements of fluid velocity in the perfusion pipette}

To quantify the SS exerted on RBCs when they pass through the narrow perfusion pipette, the fluid velocity in the perfusion pipette was measured with Photron-FASTCAM high-speed camera at 250 1000 frames/sec. ImageJ analysis software with MTrackJ plugin was used to quantify the center line red blood cell velocity (Vmax), the maximum red blood cell velocity in laminar flow parabolic profile. The mean fluid velocity (Vmean) is calculated as 
$V$ mean $=V \max / 2\left(1-8 / R^{2}\right),{ }^{21}$ and shear rate is calculated as $8 V$ mean $/ 2 R$, where $R$ is the radius of the certain cross section of a pipette. The power trendline in Excel (Microsoft office) was used for regression analysis.

\section{Solutions and reagents}

Mammalian Ringer's solution was used for the experiments. The composition of the mammalian Ringer's solution was (mM) $132 \mathrm{NaCl}, 4.6 \mathrm{KCl}, 2 \mathrm{CaCl}_{2}, 1.2 \mathrm{MgSO}_{4}, 5.5$ glucose, $5.0 \mathrm{NaHCO}_{3}, 20 \mathrm{~N}-2$ hydroxyethylpiperazine-N'-2-ethanesulfonic acid (HEPES) and Na-HEPES pH 7.4. Albumin-Ringer's solution (BSA, $10 \mathrm{mg} / \mathrm{ml}$ ) was used as control solution. Fura-2 AM was purchased from Life Technologies, Grand Island, NY. DAF-2 DA was purchased from Sigma, St. Louis, MO. All the fluorescent dyes were prepared in DMSO for stock solution and at least 1:1000 dilution was made for the final working solutions. All of the perfusates containing the test reagents were freshly prepared before each cannulation.

\section{Data analysis and statistics}

All values are means \pm SE. Paired t-test was used for paired data analysis. ANOVA was used to compare data between groups. A probability value of $\mathrm{P}<0.05$ was considered statistically significant. 


\section{RESULTS}

\section{Quantification and modulation of wall shear stress in individually perfused mesenteric rat venules}

Wall SS is the product of shear rate and viscosity of the moving fluid. To quantify the SS in individually perfused venules, we measured the viscosity of each perfusate using cone/plate viscometer and calculated the shear rate by measuring velocity of each perfusate under different perfusion pressure using high-speed camera. Fig $1 \mathrm{~A}$ shows one of the images captured at 500 frames per second in a blood perfused vessel. Viscosity of each perfusate was measured at shear rate of $225 \mathrm{~s}^{-1}$ at $37^{\circ} \mathrm{C}$. The mean viscosity of whole blood from 9 rats with mean hematocrit at $45.0 \pm 0.74 \%$ was $4.7 \pm 0.12 \mathrm{cP}$. The viscosity of rat plasma was $1.2 \pm 0.04 \mathrm{cP}(n=5)$, and $10 \%$ Dextran 70 in $1 \%$ BSA solution was 4.6 $\pm 0.13 \mathrm{cP}(n=3)$. Since each venule selected for the experiment has relatively constant diameter ( $\square 40 \square \mathrm{m}$ ), we observed a linear relationship between the net perfusion pressure (perfusion pressure minus balance pressure) and shear rate with each perfusate $(n=3$ per group, Fig 1B). By multiplying the measured viscosity, Fig 1D demonstrates the relationship between the net perfusion pressure and wall shear stress with each perfusate.

Only blood flow generated changes of shear stress induce transient increases in EC $\left[\mathrm{Ca}^{2+}\right]_{i}$ in individually perfused venules

Studies in cultured ECs and arterioles indicated that intracellular $\mathrm{Ca}^{2+}$ plays an important role in wall SS-mediated responses in vascular function. ${ }^{6,22}$ In this study we first investigated the changes in venular endothelial $\left[\mathrm{Ca}^{2+}\right]$ in response to the changes of wall SS generated by different perfusates. The mean endothelial $\left[\mathrm{Ca}^{2+}\right] \mathrm{i}$ was $57 \pm 4 \mathrm{nM}$ when 
the vessel was perfused with Ringer's solution containing $10 \mathrm{mg} / \mathrm{ml}$ of BSA at 1.1 Pa steady SS. Then the same vessel was recannulated with a micropipette containing whole blood. When the SS was increased from 0 to $1.1 \mathrm{~Pa}$ or $3.2 \mathrm{~Pa}, \mathrm{EC}\left[\mathrm{Ca}^{2+}\right]$ transiently increased to $206 \pm 14 \mathrm{nM}$ and $315 \pm 22 \mathrm{nM}$, respectively ( $\mathrm{n}=5$ per group). Fig $2 \mathrm{~A}$ shows the changes in EC $\left[\mathrm{Ca}^{2+}\right]$ from a representative experiment. However, the identical magnitude changes in SS utilizing albumin-Ringer's solution, plasma, or 10\% Dextran70 perfusate did not cause significant changes in endothelial $\left[\mathrm{Ca}^{2+}\right] \mathrm{i}($ Fig 2B-D).

\section{Both blood and plasma perfused venules show the magnitude of shear-dependent EC NO production}

Among currently reported SS-induced vasoactive factors, NO released from ECs is considered as the most important factor in the regulation of vascular function. In venular microvessels, as demonstrated in previous studies from our group ${ }^{23}$ and others, ${ }^{24}$ basal NO plays anti-inflammatory roles by preventing leukocyte adhesion and platelet aggregation, whereas inflammatory stimuli-induced NO plays a pro-inflammatory role and increases microvessel permeability. ${ }^{25}$ In this study, we further investigated the NO responses to the changes in SS in both blood and plasma perfused vessels. Using the NOindicator, DAF-2, and fluorescence imaging, we found that changes of SS induced magnitude-dependent increases in NO production in both plasma and whole blood perfused vessels. When the SS increased from 0 to $1.1 \mathrm{~Pa}$ and 0 to $3.2 \mathrm{~Pa}$, the $\mathrm{NO}$ production rate was $0.19 \pm 0.02 \mathrm{AU} / \mathrm{min}$ and $0.48 \pm 0.10 \mathrm{AU} / \mathrm{min}$ in plasma perfused vessels $(n=3)$, and $0.13 \pm 0.02 \mathrm{AU} / \mathrm{min}$ and $0.24 \pm 0.02 \mathrm{AU} / \mathrm{min}$ in blood perfused vessels $(n=3$, Figure $3 \mathrm{~A}$ and $3 \mathrm{~B}$ ). The higher NO-dependent DAF-2 signal detected in plasma-perfused 
vessels as compared to blood perfused vessels may be attributed to the scavenging of NO by RBCs.

\section{Shear stress-induced eNOS activation}

To correlate SS-induced NO production with eNOS activation status, we examined eNOS phosphorylation at Serine 1177 and Threonine 495 under the same experimental conditions as those in which NO was measured using immunofluorescence staining and confocal imaging ( $n=3$ per group). eNOS-Ser ${ }^{1177}$ phosphorylation showed similar responses to blood and plasma perfusion-generated SS, and the levels of changes were shear magnitude-dependent (Fig 4A-B). However, shear magnitude-dependent eNOS$T h r^{495}$ dephosphorylation only occurred in blood perfused vessels, and no significant changes were found in plasma perfused vessels (Fig 4C-D).

\section{ATP released from RBCs is responsible for shear stress-induced transient increases in $\mathrm{EC}\left[\mathrm{Ca}^{2+}\right]_{i}$ in individually perfused venules}

One of the major differences between whole blood and plasma perfused vessels is the presence of blood cells. Since the majority of the blood cells are RBCs, we examined whether the presence of RBCs contributes to SS-induced changes in $\mathrm{EC}\left[\mathrm{Ca}^{2+}\right] \mathrm{i}$. Results showed that perfusion of vessels with $1 \%$ albumin-Ringer perfusate containing $40 \%$ (volume) of RBCs resulted a similar pattern of shear-induced increases in $\mathrm{EC}\left[\mathrm{Ca}^{2+}\right]_{i}$ to those in whole blood perfused vessels. EC $\left[\mathrm{Ca}^{2+}\right]$ i transiently increased from $76 \pm 6 \mathrm{~nm}$ to $218 \pm 10 \mathrm{nM}$ and $314 \pm 30 \mathrm{nM}$ when SS increased from $0 \mathrm{~Pa}$ to $1.1 \mathrm{~Pa}$ and $3.2 \mathrm{~Pa}$, respectively ( $n=3$, Fig $5 A$ and $B)$. 
Several studies have indicated that RBCs release ATP in response to shear, and that a pannexin 1 channel on their membrane serves as a mechanical sensor and is responsible for the ATP release. ${ }^{9,} 26$ We examined shear-induced ATP release from RBCs using a coneplate viscometer to generate SS and luciferin/luciferase assay to measure ATP. We found that SS induced magnitude-dependent ATP release from RBCs, and preincubation of RBCs with pannexin 1 inhibitor, carbenoxolone $(100 \mu M)$, blocked the ATP release $(n=3$, Fig. 5C). Furthermore, perfusing microvessels with carbenoxolone (100 $\mu \mathrm{M})$ treated RBCs also abolished SS-induced increases in EC $\left[\mathrm{Ca}^{2+}\right]_{i}(\mathrm{Fig} 5 \mathrm{D})$. The mean EC $\left[\mathrm{Ca}^{2+}\right]_{i}$ were 71 $\pm 21 \mathrm{nM}$ and $80 \pm 23 \mathrm{nM}$ when SS was increased from 0 to $1.1 \mathrm{~Pa}$ and $3.2 \mathrm{~Pa}$, respectively (Fig 5B).

\section{Shear stress-induced endothelial gap formation}

Our previous study demonstrated that the magnitude of the accumulation of fluorescent microspheres (FMs) at junctions between ECs is closely correlated with the degree of endothelial gap formation and increased microvessel permeability. ${ }^{20}$ To determine whether the change of SS results in increased microvessel permeability, FMs $(100 \mathrm{~nm})$ were added to the blood or plasma perfusate to mark the endothelial gap formation upon changing SS. We found that only in blood perfused vessels, increase of SS from 0 to $3.2 \mathrm{~Pa}$ induced significant accumulation of perfused FMs at endothelial junctions and the FI of accumulated FMs was $5.1 \pm 0.5$ times the $0 \mathrm{~Pa}$ control (Fig $6 \mathrm{~A}$ right panel). The identical experimental procedures performed in plasma perfused vessels showed no such effect (Fig 6A left panel, $n=3)$. RBC solutions ( $40 \%$ hematocrit) also showed similar effects to blood perfused vessels, with the FI of accumulated FMs being $6.9 \pm 1.1$ times the 0 Pa control (Fig 6B left, 
$n=5$ ). When the vessels were perfused with $40 \%$ carbenoxolone-treated RBCs, the FI of accumulated FMs was significantly decreased to $1.5 \pm 0.1$ times the $0 \mathrm{~Pa}$ control value (Fig $6 B$ right, $n=6)$.

\section{Additional shear stress exerted on RBCs when they pass through the perfusion pipette}

The identification of the roles of RBCs in shear-induced EC signaling and permeability changes led us a further evaluation of the shear forces the RBCs experienced in individually perfused microvessels. It has been reported that constricted RBCs from a wide channel to a narrow channel in microfluidic devices generated sudden increase of SS on the RBCs, which triggers the ATP release from the RBCs. ${ }^{9}{ }^{10}$ Based on those observations, our single vessel perfusion technique essentially replicates the shear conditions of the microfluidic devices with even larger magnitude changes in SS when the RBCs pass through the narrow perfusion pipette during whole blood or RBC perfusion. Therefore, under our experimental conditions, RBCs in the perfusate not only experienced the wall shear in the vessel lumen, but also experienced a greater transient changes in shear when passing through the tip of the perfusion pipette. We hypothesized that shear-induced ATP release from RBCs contribute to the shear-dependent increases in endothelial $\left[\mathrm{Ca}^{2+}\right]$ i. To quantify the shear that RBCs experienced when they pass through the perfusion pipette, we measured the RBC velocity near the pipette tip as the inner diameter narrowed (Fig 7A). Fig 7 B-C shows the mean velocity profile of the RBCs within the perfusion pipette as a function of the distance from the pipette tip, a factor linearly associated with the changes in pipette diameter. When the wall shear conditions were increased from 0 to $1.1 \mathrm{~Pa}$ and 3.2 
$\mathrm{Pa}$, respectively, the sharpest change in RBC velocity started at around $150 \mu \mathrm{m}$ from the upstream of the pipette tip ( $n=3$ for each group) and the maximum shear rate was about 10 time higher than they experienced in the vessel lumen, which may greatly contribute to ATP-induced increases in endothelial $\left[\mathrm{Ca}^{2+}\right]$. 


\section{DISCUSSION}

Our studies by direct comparisons of intact microvascular responses to SS generated by cell-free fluids, such as plasma or solutions containing Dextran 70, with SS generated by blood flow demonstrated that under in vivo conditions, the changes of blood flow affect endothelium not only by fluid-generated wall SS, but also by shear-induced releases of ATP from RBCs. Each component contributes to different EC signaling and affects important vascular functions. Our results indicated that an increase of fluid-generated wall SS induces increased EC NO production, which does not involve an increase in endothelial $\left[\mathrm{Ca}^{2+}\right]$ i. However, under in vivo conditions, i.e. in blood perfused vessels, the increased shear exerted on RBCs induces shear-magnitude dependent ATP release. This SSdependent ATP release from RBCs causes increases in $\mathrm{EC}\left[\mathrm{Ca}^{2+}\right]$ and endothelial gap formation, an indication of increases in microvessel permeability. By linking the effects of wall SS on endothelium with the effects of shear-induced ATP release from RBCs on endothelium, our study provided new insight about how changes of blood flow affect vascular functions in vivo.

\section{Mechanisms of shear stress-induced increases in $\mathrm{EC}\left[\mathrm{Ca}^{2+}\right]_{i}$ : the role of shear- induced ATP release from RBCs}

It has been controversial for decades whether exposure of EC to flow is accompanied by elevated cytoplasmic $\left[\mathrm{Ca}^{2+}\right]_{\mathrm{i}}$, 27 Our results provided the first evidence in intact microvessels that the changes of SS in plasma or similar viscous dextran-albumin-Ringerperfused vessels do not cause increases in $\mathrm{EC}\left[\mathrm{Ca}^{2+}\right]$, and the SS-induced increases in $E C\left[\mathrm{Ca}^{2+}\right]$ i only occur in blood perfused microvessels and require the presence of RBCs. 
Our experiments that measured SS-induced ATP release from RBCs in vitro in combination with quantifications of SS exerted on RBCs through single vessel perfusion also provided mechanistic explanations for RBC involved SS-dependent increases in $\mathrm{EC}\left[\mathrm{Ca}^{2+}\right]_{\mathrm{i}}$ in intact microvessels.

Previous studies using an in vitro microfluidic approach or microbore tubing reported that RBCs release ATP upon exposure to increased SS or cell deformation, and the amount of ATP released was directly proportional to the number of RBCs and the magnitude of the

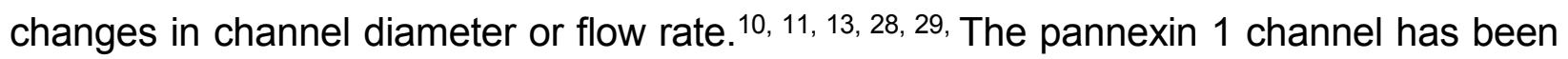
reported to act as a mechanical sensor on the membrane surface of RBCs and to mediate ATP release, as inhibition of pannexin 1 attenuates SS-induced ATP release. ${ }^{9,}{ }^{26}$ However, these studies did not involve endothelial cells, and to date the effect of SS-induced ATP release from RBCs on the endothelium has not been well explored, especially in intact microvessels. Our experiments using cone-plate viscometry to generate SS on RBCs in the presence and absence of a pannexin 1 inhibitor further confirmed the role of the pannexin 1 channel in the shear-dependent ATP release from RBCs. We previously demonstrated that perfusing intact venules with ATP $(10 \square \mathrm{M})$ induced transient increases in $\mathrm{EC}\left[\mathrm{Ca}^{2+}\right] \mathrm{i}$ and microvessel Lp. ${ }^{30}$ In this study, we not only quantified the wall SS with different perfusates when the endothelial cell $\left[\mathrm{Ca}^{2+}\right]_{i}$ and NO production were measured, but also analyzed the additional SS exerted on RBCs via micropipet perfusion of individual microvessels. In addition to changes in shear within the lumen of the perfused microvessel, RBCs experienced marked increases in SS when they passed through the narrow tip of the perfusion pipette (Fig 7), The shear effects of micropipette perfusion was similar to the narrow constriction design used in microfluidic channel studies, in which shear-induced 
ATP release was detected from RBCs passing through the constrictions. ${ }^{9}, 10$ Based on those studies and our in vitro measurements of SS-induced ATP release from RBCs, we predict that under our experimental conditions the increased SS on RBCs caused by moving through narrow perfusion pipette and increased fluid velocity to raise wall SS induced significant amount of ATP release from RBCs, and this increased ATP release contributes to the observed increases in $\mathrm{EC}\left[\mathrm{Ca}^{2+}\right]_{i}$ in blood perfused vessels. Our experiments using carbenoxolone, a selective inhibitor of pannexin 1, which abolished SSinduced ATP release in vitro and SS-dependent increases in EC $\left[\mathrm{Ca}^{2+}\right]$ in blood perfused intact microvessels (Fig 5C), provide further evidence that SS-induced increases in EC $\left[\mathrm{Ca}^{2+}\right]_{i}$ are directly associated with ATP released from RBCs.. Although some cultured cell studies reported SS-induced ATP release from ECs resulting in increases in EC $\left.\left[\mathrm{Ca}^{2+}\right]\right]^{31}$ based on our EC $\left[\mathrm{Ca}^{2+}\right]$ measurements in plasma and dextran 70-perfused vessels, any SS-induced EC released ATP was insufficient to cause increases in EC $\left[\mathrm{Ca}^{2+}\right]$ in intact microvessels.

\section{Shear stress induced EC NO production and eNOS activation}

Although it has been well recognized that the changes of SS induce NO production from ECs, the signaling mechanisms remain unclear, especially because there is a lack of in vivo evidence. Prior in vitro studies on the role of $\mathrm{Ca}^{2+}$ in SS-mediated NO production have been controversial. Some studies reported that SS-induced NO was independent of external $\mathrm{Ca}^{2+}$, but was mediated by increases in cytoplasmic $\mathrm{Ca}^{2+}$ via $\mathrm{Ca}^{2+}$ release from intracellular stores. ${ }^{6,32}$ Others reported that flow-induced NO release was blocked by removal of extracellular $\mathrm{Ca}^{2+}$ or the application of $\mathrm{Ca}^{2+}$ and calmodulin (CaM) antagonists. ${ }^{7}$ 
${ }^{33}$ There are also studies reported that flow-induced NO occurred independently of changes in intracellular $\left[\mathrm{Ca}^{2+}\right] \mathrm{i} .{ }^{34}$ These inconsistencies could be the result of the differences in cultured cell species, ATP concentrations in culture media, and the applied flow patterns. A study conducted in rabbit lungs indicated that mechanical force-induced NO synthesis requires ATP release from RBCs. ${ }^{12}$ Our measurements of both $\mathrm{NO}$ and endothelial $\left[\mathrm{Ca}^{2+}\right]_{\mathrm{i}}$ responses in blood and plasma perfused intact microvessels demonstrated that similar increases in fluid generated wall SS, with and without RBCs, induce increases in NO production. However, only increasing SS with perfusion solutions with substantial numbers of RBCs increased endothelial $\left[\mathrm{Ca}^{2+}\right]_{\mathrm{i}}$. These observations indicate that SS-induced NO can be independent of increases in endothelial $\left[\mathrm{Ca}^{2+}\right]$. The increases in SS-induced EC $\left[\mathrm{Ca}^{2+}\right]_{i}$ observed in blood-perfused microvessels are dependent on the magnitude of SS, but they are attributed to EC responses to shear-induced releases of ATP from RBCs. Our result that blocking ATP release from RBCs abolished SS-induced increases in endothelial $\left[\mathrm{Ca}^{2+}\right]$ s supports this conclusion.

To further evaluate the $\mathrm{Ca}^{2+}$-independence of SS-induced NO production,, we assessed the activation status of eNOS following the same experimental procedures as those EC $\left[\mathrm{Ca}^{2+}\right]_{i}$ and NO were measured using immunofluorescence staining and confocal imaging. Two separate amino acid residues are reported to be particularly important in regulating eNOS activity: a serine residue Ser ${ }^{1177}$ and a threonine residue Thr ${ }^{495} \cdot{ }^{35}$ Cultured EC and isolated protein studies indicated that eNOS-Ser ${ }^{1177}$ phosphorylation represents a $\mathrm{Ca}^{2+}$-independent regulatory mechanism for activation of eNOS, ${ }^{36}$ but the dephosphorylation of $\mathrm{Thr}^{495}$-associated eNOS activation depends on increased EC $\left[\mathrm{Ca}^{2+}\right]_{\mathrm{i} .}{ }^{37}$ Our results that SS-induced eNOS-Ser ${ }^{1177}$ phosphorylation occurred in both 
plasma and whole blood perfused vessels, while SS-induced eNOSThr ${ }^{495}$ dephosphorylation only occurred in blood perfused microvessels, are consistent with the molecular characterization of each of the eNOS activation sites. These results further support the hypothesis that the changes in wall SS in the absence of RBCs activate eNOS via a $\mathrm{Ca}^{2+}$-independent mechanism, indicating the wall SS-induced NO production can be independent from increases in $\mathrm{EC}\left[\mathrm{Ca}^{2+}\right]$. In the presence of RBCs, the SS-induced activation of eNOS involves both $\mathrm{Ca}^{2+}$ dependent and independent mechanisms with $\mathrm{Ca}^{2+}$ dependent eNOS activation being the result of ATP-induced increases in EC $\left[\mathrm{Ca}^{2+}\right]$.

\section{Shear stress and microvessel permeability}

Although studies in individually perfused microvessels in both frog and rat mesenteries have consistently demonstrated flow-dependent increases in permeability to small solutes, such as $\mathrm{K}^{+}$and sodium fluorescein, ${ }^{38,39}$ the results of flow-induced changes in permeability to macromolecules and fluid (Lp) remain conflicting. ${ }^{14-16,40}$ Studies using isolated perfused pig coronary venules reported perfusion rate-dependent increases in permeability to albumin. ${ }^{40}$ In individually perfused frog mesenteric venules, one study showed that the initial measured $L p$ value was correlated with the blood flow rate prior to the vessel cannulation, ${ }^{15}$ but others using the similar techniques reported no changes in Lp upon changes in flow velocity or wall SS. ${ }^{14,16,38}$ Based on the measurements of flow-induced permeability to small solutes, the changes occurred rapidly. ${ }^{38,39}$ It is a great technical challenge to quantitatively measure SS-induced hydraulic permeability following the traditional single vessel perfusion and occlusion technique. ${ }^{21}$ To date, all of studies using single vessel perfusion techniques were conducted in the absence of RBCs (i.e. $<1 \%$ RBCs 
which serve as velocity markers), and therefore assess only cell-free fluid generated wall SS. To overcome the technical difficulties of assessing SS-induced permeability to fluid and macromolecules, especially in the presence of RBCs, we directly evaluated EC junctional changes in response to changed SS using FMs as marker to assess EC gap formation. ${ }^{20}$ Our previous studies demonstrated that when FMs (100 nm diameter) were perfused into platelet activating factor (PAF) stimulated rat venules, the amount of accumulated FMs at EC junctions serves as an index for the dimension of gap formation between ECs (validated by electron micrograph), and is temporally correlated with the time course of PAF-induced Lp changes. ${ }^{20}$ Using this method, our current results demonstrated that changes of SS generated by cell-free fluid (plasma or dextran 70-BSA-solutions) showed no significant increase in accumulation of FMs at vascular walls, indicating no additional EC gap formation. However, changes of SS generated with perfusion solutions containing $40 \%$ RBCs increased EC gap formation, reflected by increased FM accumulation at EC junctions. Significantly, this increased junctional accumulation of FMs was abolished when RBCs were pre-treated with a pannexin 1 inhibitor, suggesting endothelial gap formation is directly linked with RBC released ATP and ATP-induced increases in EC $\left[\mathrm{Ca}^{2+}\right]$. SSinduced NO production alone (in the absence of RBC released ATP) showed no effect on EC $\left[\mathrm{Ca}^{2+}\right]$ i or EC gap formation. Based on our previous studies, all agonist-induced permeability increases are associated with increases in $\mathrm{EC}\left[\mathrm{Ca}^{2+}\right]_{\mathrm{i}}{ }^{8,}, 18$ Our present results suggest that the agonist-induced $\mathrm{Ca}^{2+}$-dependent permeability regulation also applies to RBC associated shear force-induced changes in microvessel barrier function.

\section{Summary}


The main contribution of this study is that we demonstrated the response of endothelial cells to changes in SS in intact venules. Our results revealed that under in vivo conditions, ECs not only respond to fluid generated wall SS, but also to ATP released by RBCs in response to shear and that this ATP plays important roles in altering EC integrity and vascular permeability. The studies presented in this paper provide the basic mechanisms that may explain and predict the roles of changing SS in different vasculature under both physiological and pathological conditions. Variations in vascular geometry involving narrowed regions, such as blood entry from small capillary to venules or at vascular converging points, may expose the RBCs to high SS, cause the release of ATP, and increase $\mathrm{EC}\left[\mathrm{Ca}^{2+}\right]_{i}$ and EC gap formation in downstream vessels. Under pathological conditions, such as arterial stenosis due to local atherosclerotic plaque formation, or mechanical injury-induced local vascular remodeling and cell proliferation, may cause similar responses which would exacerbate local inflammation and vascular damage. Although further validation under those conditions is needed, our observations indicate that the effect of SS-induced ATP release from RBCs should not be underestimated or omitted when SS-induced changes in EC function is studied. 


\section{REFERENCE}

1. Resnick N, Yahav H, Shay-Salit A, Shushy M, Schubert S, Zilberman LC, Wofovitz E. Fluid shear stress and the vascular endothelium: for better and for worse. Prog Biophys Mol Biol. 2003;81(3):177-199.

2. Pang Z, Antonetti DA, Tarbell JM. Shear stress regulates HUVEC hydraulic conductivity by occludin phosphorylation. Ann Biomed Eng. 2005;33(11):15361545.

3. Sill HW, Chang YS, Artman JR, Frangos JA, Hollis TM, Tarbell JM. Shear stress increases hydraulic conductivity of cultured endothelial monolayers. Am J Physiol. 1995;268(2 Pt 2):H535-543.

4. Lakshminarayanan S, Gardner TW, Tarbell JM. Effect of shear stress on the hydraulic conductivity of cultured bovine retinal microvascular endothelial cell monolayers. Curr Eye Res. 2000;21(6):944-951.

5. Morigi M, Zoja C, Figliuzzi M, Foppolo M, Micheletti G, Bontempelli M, Saronni M, Remuzzi G, Remuzzi A. Fluid shear stress modulates surface expression of adhesion molecules by endothelial cells. Blood. 1995;85(7):1696-1703.

6. Ando J, Komatsuda T, Kamiya A. Cytoplasmic calcium response to fluid shear stress in cultured vascular endothelial cells. In Vitro Cell Dev Biol. 1988;24(9):871-877.

7. Kuchan MJ, Frangos JA. Role of calcium and calmodulin in flow-induced nitric oxide production in endothelial cells. Am J Physiol. 1994;266(3 Pt 1):C628-636.

8. Curry FR, Adamson RH. Vascular permeability modulation at the cell, microvessel, or whole organ level: towards closing gaps in our knowledge. Cardiovasc Res. 2010;87(2):218-229.

9. Forsyth AM, Wan J, Owrutsky PD, Abkarian M, Stone HA. Multiscale approach to link red blood cell dynamics, shear viscosity, and ATP release. Proc Natl Acad Sci U S A. 2011;108(27):10986-10991.

10. Wan J, Ristenpart WD, Stone HA. Dynamics of shear-induced ATP release from red blood cells. Proc Natl Acad Sci U S A. 2008;105(43):16432-16437.

11. Edwards J, Sprung R, Sprague R, Spence D. Chemiluminescence detection of ATP release from red blood cells upon passage through microbore tubing. Analyst. 2001;126(8):1257-1260.

12. Sprague RS, Ellsworth ML, Stephenson AH, Lonigro AJ. ATP: the red blood cell link to NO and local control of the pulmonary circulation. Am J Physiol. 1996;271(6 Pt 2):H2717-2722. 
13. Moehlenbrock MJ, Price AK, Martin RS. Use of microchip-based hydrodynamic focusing to measure the deformation-induced release of ATP from erythrocytes. Analyst. 2006;131(8):930-937.

14. Neal CR, Bates DO. Measurement of hydraulic conductivity of single perfused Rana mesenteric microvessels between periods of controlled shear stress. J Physiol. 2002;543(Pt 3):947-957.

15. Williams DA. A shear stress component to the modulation of capillary hydraulic conductivity (Lp). Microcirculation. 1996;3(2):229-232.

16. Adamson RH, Sarai RK, Altangerel A, Clark JF, Weinbaum S, Curry FE. Microvascular permeability to water is independent of shear stress, but dependent on flow direction. Am J Physiol Heart Circ Physiol. 2013;304(8):H1077-1084.

17. Zweifach BW, Lipowsky HH. Quantitative studies of microcirculatory structure and function. III. Microvascular hemodynamics of cat mesentery and rabbit omentum. Circ Res. 1977;41(3):380-390.

18. He $\mathrm{P}$, Zhang $\mathrm{X}$, Curry FE. Ca2+ entry through conductive pathway modulates receptor-mediated increase in microvessel permeability. Am J Physiol. 1996;271(6 Pt 2):H2377-2387.

19. Zhu L, He P. Platelet-activating factor increases endothelial [Ca2+]i and NO production in individually perfused intact microvessels. Am J Physiol Heart Circ Physiol. 2005;288(6):H2869-2877.

20. Jiang $Y$, Wen $K$, Zhou $X$, Schwegler-Berry D, Castranova V, He P. Threedimensional localization and quantification of PAF-induced gap formation in intact venular microvessels. Am J Physiol Heart Circ Physiol. 2008;295(2):H898-906.

21. Michel CC, Mason JC, Curry FE, Tooke JE, Hunter PJ. A development of the Landis technique for measuring the filtration coefficient of individual capillaries in the frog mesentery. Q J Exp Physiol Cogn Med Sci. 1974;59(4):283-309.

22. Ungvari Z, Sun D, Huang A, Kaley G, Koller A. Role of endothelial [Ca2+]i in activation of eNOS in pressurized arterioles by agonists and wall shear stress. Am J Physiol Heart Circ Physiol. 2001;281(2):H606-612.

23. Xu S, Zhou X, Yuan D, Xu Y, He P. Caveolin-1 scaffolding domain promotes leukocyte adhesion by reduced basal endothelial nitric oxide-mediated ICAM-1 phosphorylation in rat mesenteric venules. Am J Physiol Heart Circ Physiol. 2013;305(10):H1484-1493.

24. Granger DN, Kubes P. Nitric oxide as antiinflammatory agent. Methods Enzymol. 1996;269:434-442. 
25. Zhou X, He P. Endothelial [Ca2+]i and caveolin-1 antagonistically regulate eNOS activity and microvessel permeability in rat venules. Cardiovasc Res.87(2):340-347.

26. Locovei S, Bao L, Dahl G. Pannexin 1 in erythrocytes: function without a gap. Proc Natl Acad Sci U S A. 2006;103(20):7655-7659.

27. Mo M, Eskin SG, Schilling WP. Flow-induced changes in Ca2+ signaling of vascular endothelial cells: effect of shear stress and ATP. Am J Physiol. 1991;260(5 Pt 2):H1698-1707.

28. Fischer DJ, Torrence NJ, Sprung RJ, Spence DM. Determination of erythrocyte deformability and its correlation to cellular ATP release using microbore tubing with diameters that approximate resistance vessels in vivo. Analyst. 2003;128(9):11631168.

29. Sprung R, Sprague R, Spence D. Determination of ATP release from erythrocytes using microbore tubing as a model of resistance vessels in vivo. Anal Chem. 2002;74(10):2274-2278.

30. He P, Curry FE. Differential actions of cAMP on endothelial [Ca2+]i and permeability in microvessels exposed to ATP. Am J Physiol. 1993;265(3 Pt 2):H1019-1023.

31. Yamamoto K, Furuya K, Nakamura M, Kobatake E, Sokabe M, Ando J. Visualization of flow-induced ATP release and triggering of $\mathrm{Ca} 2+$ waves at caveolae in vascular endothelial cells. J Cell Sci.124(Pt 20):3477-3483.

32. Shen J, Luscinskas FW, Connolly A, Dewey CF, Jr., Gimbrone MA, Jr. Fluid shear stress modulates cytosolic free calcium in vascular endothelial cells. Am J Physiol. 1992;262(2 Pt 1):C384-390.

33. Buga GM, Gold ME, Fukuto JM, Ignarro LJ. Shear stress-induced release of nitric oxide from endothelial cells grown on beads. Hypertension. 1991;17(2):187-193.

34. O'Neill WC. Flow-mediated NO release from endothelial cells is independent of $\mathrm{K}+$ channel activation or intracellular Ca2+. Am J Physiol. 1995;269(4 Pt 1):C863-869.

35. Fleming I, Busse R. Molecular mechanisms involved in the regulation of the endothelial nitric oxide synthase. Am J Physiol Regul Integr Comp Physiol. 2003;284(1):R1-12.

36. Fleming I, Busse R. Signal transduction of eNOS activation. Cardiovasc Res. 1999;43(3):532-541.

37. Fleming I, Fisslthaler B, Dimmeler S, Kemp BE, Busse R. Phosphorylation of

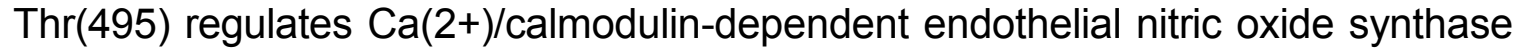
activity. Circ Res. 2001;88(11):E68-75. 
38. Montermini D, Winlove CP, Michel C. Effects of perfusion rate on permeability of frog and rat mesenteric microvessels to sodium fluorescein. J Physiol. 2002;543(Pt 3):959-975.

39. Kajimura M, Head SD, Michel CC. The effects of flow on the transport of potassium ions through the walls of single perfused frog mesenteric capillaries. J Physiol. 1998;511 ( Pt 3):707-718.

40. Yuan Y, Granger HJ, Zawieja DC, Chilian WM. Flow modulates coronary venular permeability by a nitric oxide-related mechanism. Am J Physiol. 1992;263(2 Pt 2):H641-646. 


\section{FIGURES AND FIGURE LEGENDS}

\section{Figure 1}

A

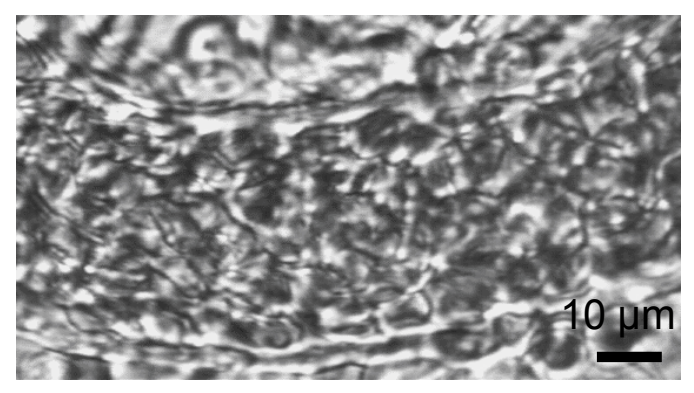

C

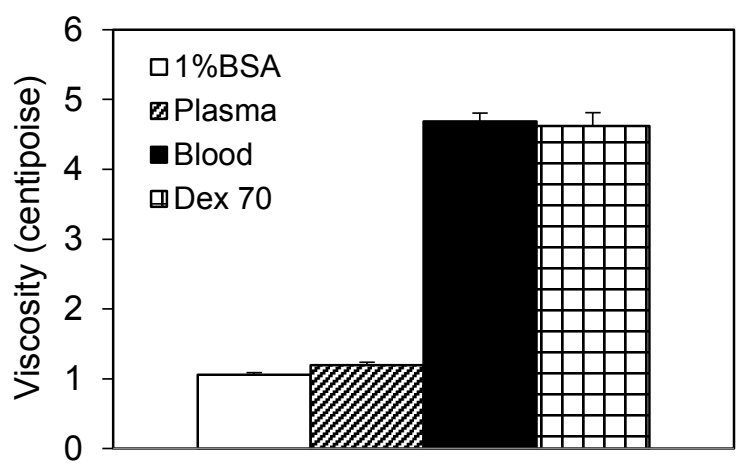

B

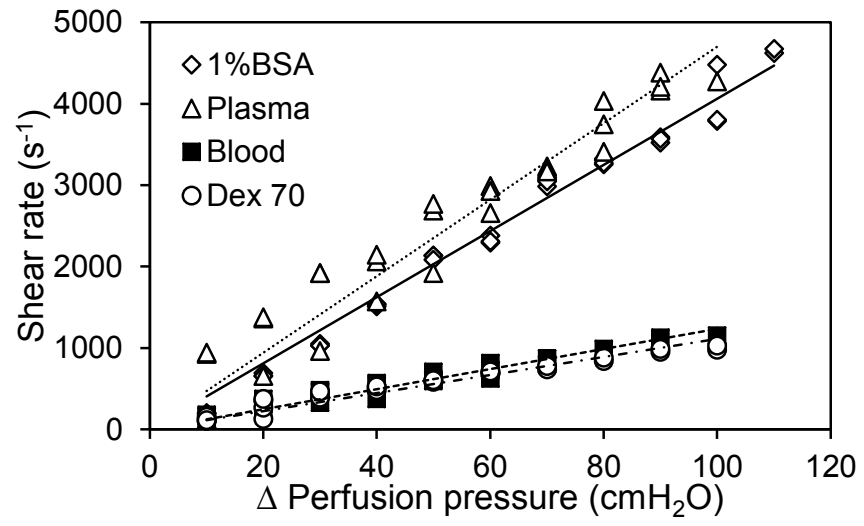

$\mathrm{D}$

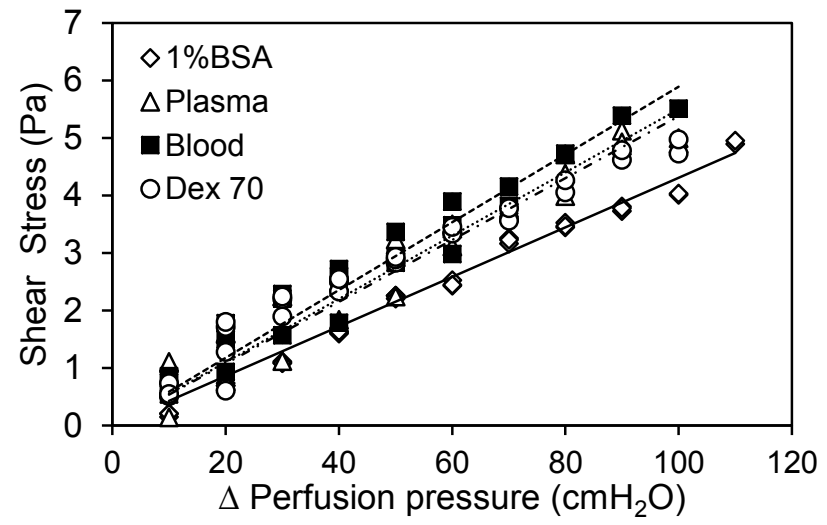

Figure 1. Shear stress measurement in intact mesenteric venules. A) High speed camera image of the blood flow in a rat mesenteric venule. B) Relationship of shear rate to perfusion pressure using different perfusate solutions. C) Summary of different perfusate viscosity measured by cone-plate viscometer $\mathrm{D})$ Relationship of shear stress to perfusion pressure using different perfusate solutions. 
Figure 2

A

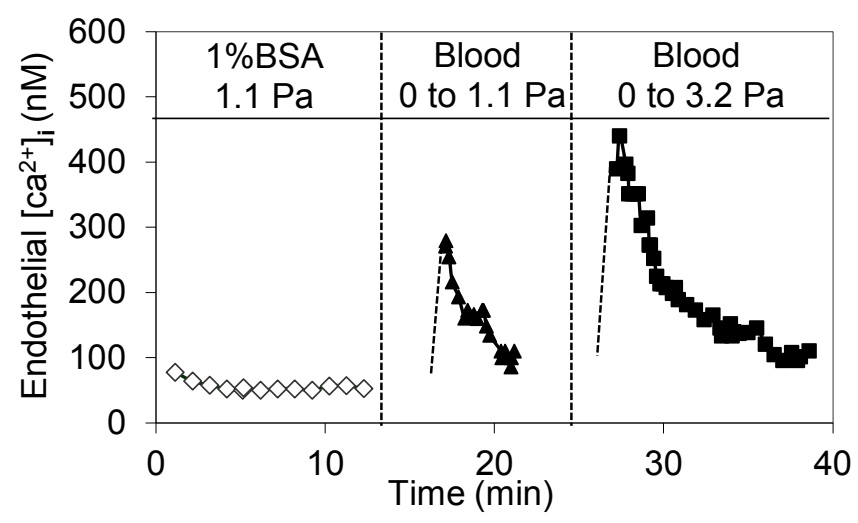

C

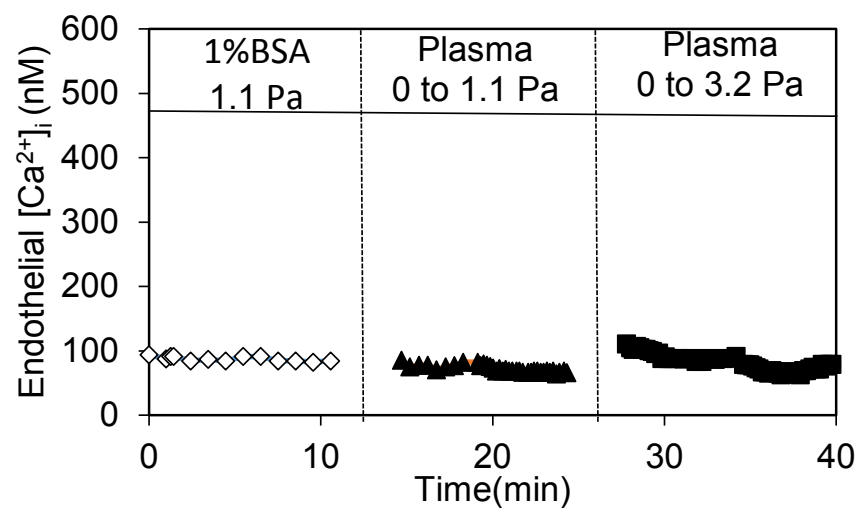

E

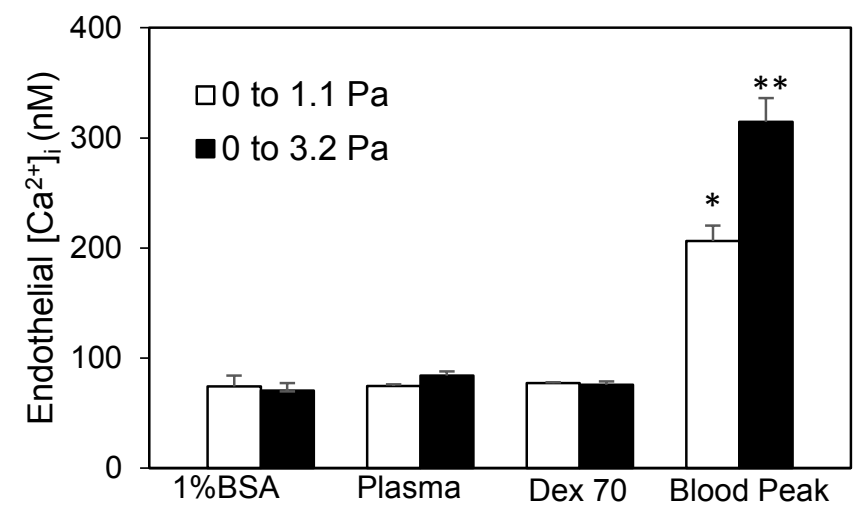

B
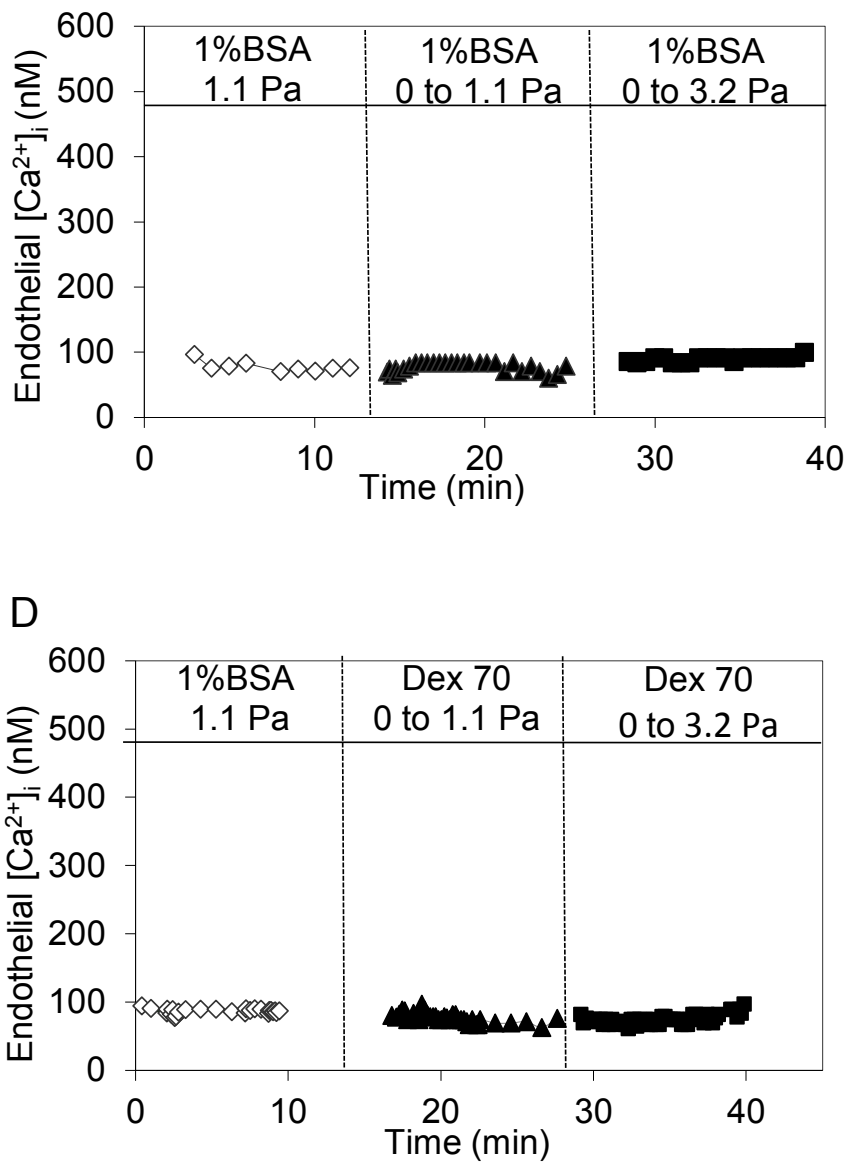
Figure 2. Blood flow generated-shear stress induced transient increase in $E C\left[\mathrm{Ca}^{2+}\right] \mathrm{i}$ in individually perfused venules. A) The time course of blood flow generated-shear stress induced EC $\left[\mathrm{Ca}^{2+}\right]$ i changes from a representative experiment. B-D) The time courses of $1 \% \mathrm{BSA}$, plasma and $10 \%$ Dextran70 generated-shear stress induced $\mathrm{EC}\left[\mathrm{Ca}^{2+}\right]_{\mathrm{i}}$ changes from respectively representative experiments E) Summary data show that only whole blood perfusion at different perfusion pressure induced shear-magnitude dependent transient EC $\left[\mathrm{Ca}^{2+}\right]$ increase ( $\mathrm{n}=5$ for the blood group, $\mathrm{n}=3$ for the other groups) * indicates a significant increase from the $1 \% \mathrm{BSA}$ control, ${ }^{* *}$ indicates a significant increase from 1.1 Pa group generated by blood perfusion 
Figure 3

A

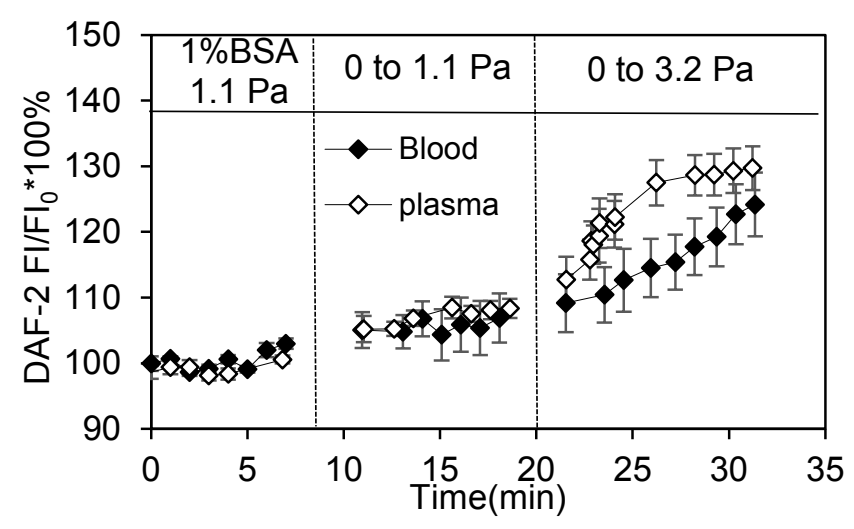

B

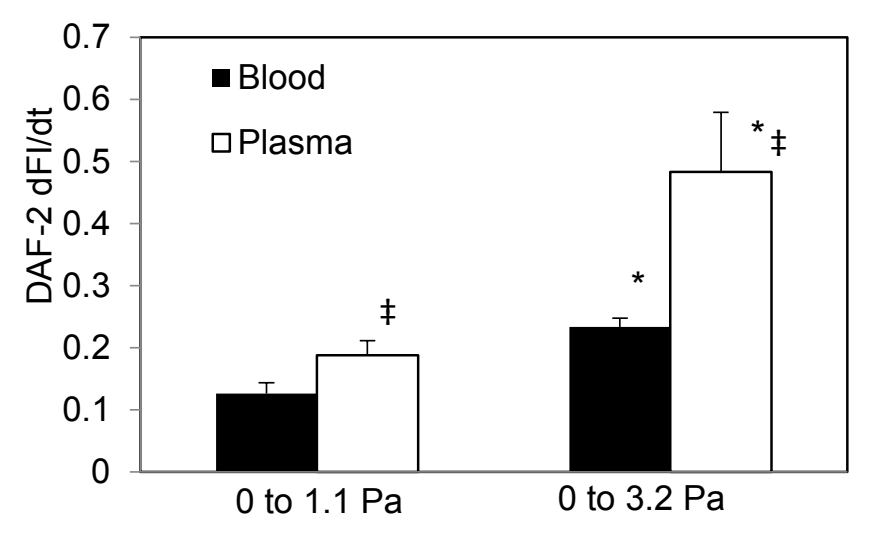

Figure 3. Shear stress-induced EC NO production in rat mesenteric venules A) Time course of representative experiments showed that both the onset of plasma and whole blood perfusion-generated sudden change of shear stress induced shear-magnitude dependent increases of EC NO production. B) Summary data from three vessels from each group showed the rate of NO production over different magnitude of shear stress. indicates a significant increase from the from the 1.1 Pa group generated by the same perfusate, $\ddagger$ indicates a significant increase from blood perfusion group at same shear stress magnitude. 
Figure 4

A

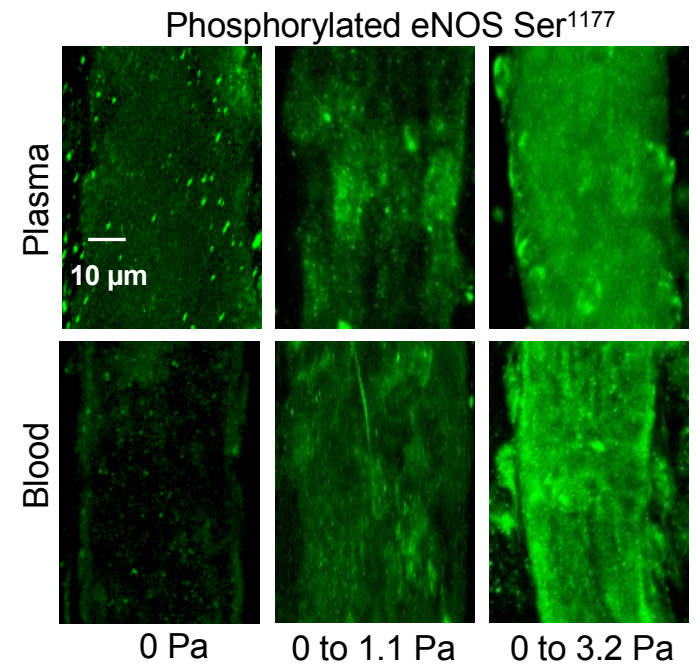

C

Phosphorylated eNOS Thr ${ }^{495}$

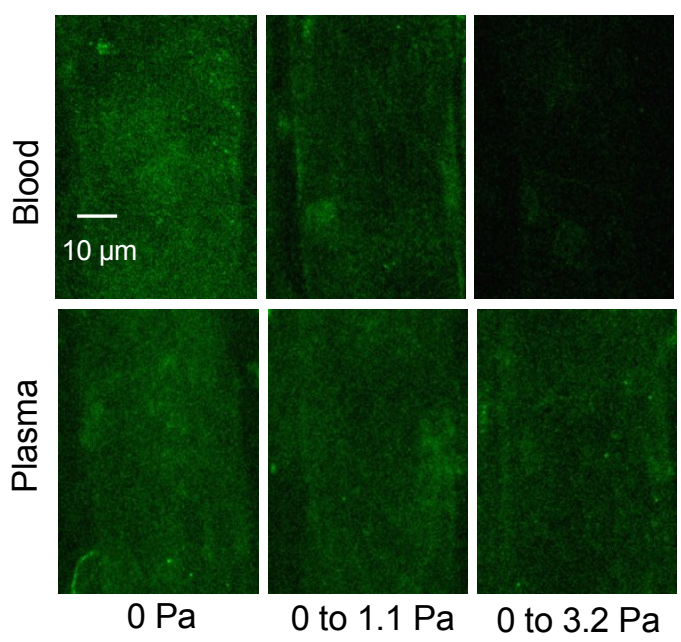

B

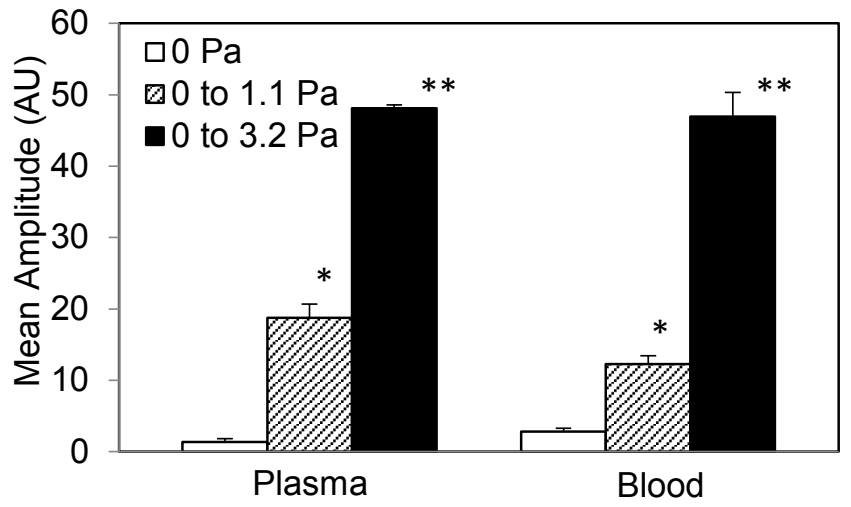

D

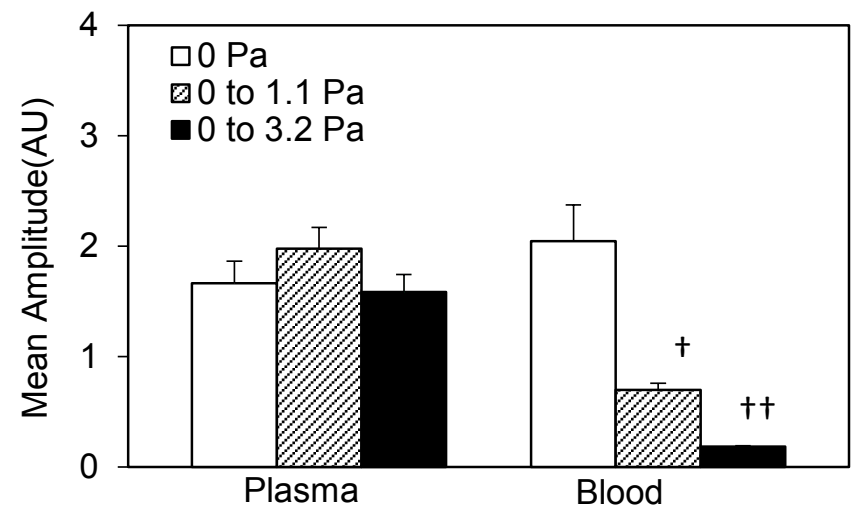


Figure 4. EC eNOS phosphorylation (eNOS-Ser ${ }^{1177}$ and eNOS- Thr ${ }^{495}$ ) in response to different magnitudes of shear stress generated by either blood or plasma perfusion in the perfused venules. A) Confocal images of shear-dependent phosphorylation of eNOS-Ser ${ }^{1177}$ in blood and plasma perfused vessels. C) Confocal images of sheardependent dephosphorylation of eNOS- Thr ${ }^{495}$ in blood perfused group but not in plasma ones. B\&D) Summary of the fluorescence changes under different experimental conditions ( $n=3$ for each group). ${ }^{*}$ indicates a significant increase from the $0 \mathrm{~Pa}$ control, ${ }^{*}$ indicates a significant increase from the lower shear stress group generated by the same perfusate, $\dagger$ indicates a significant decrease from the $0 \mathrm{~Pa}$ control. $\dagger \dagger$ indicates a significant decrease from the 1.1 Pa group generated by blood perfusion. 
Figure 5.
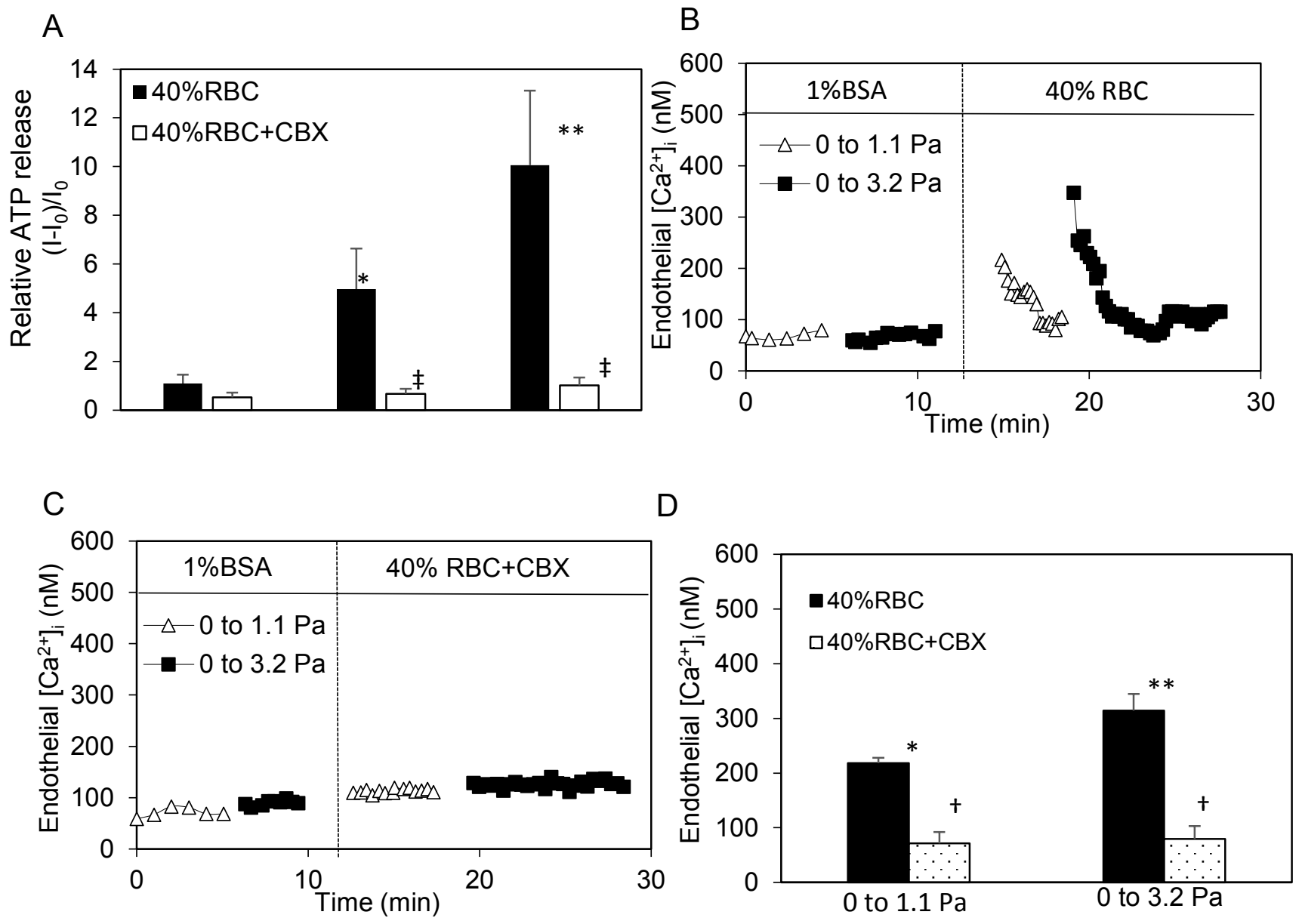
Figure 5. ATP release from erythrocytes are required for shear stress-induced transient increase in EC $\left[\mathrm{Ca}^{2+}\right]_{i}$ in individually perfused venules. A) Summary of ATP measurement from red blood cells exposing to shear stress in the presence and absence of carbenoxolone B-C) Representative experiments shows $40 \% \mathrm{RBC}$ in albumin ringer solution perfusion induced shear-magnitude dependent $E C\left[\mathrm{Ca}^{2+}\right]$ transient increase, while carbenoxolone incubated RBC solution using the same experiment procedure greatly diminished the EC $\left[\mathrm{Ca}^{2+}\right]$ increase D) Summary data show that carbenoxolone abolished the RBC solution induced EC $\left[\mathrm{Ca}^{2+}\right]$ i transient increase. ( $\mathrm{n}=3$ for each group). ${ }^{*}$ indicates a significant increase from the $1 \% \mathrm{BSA}$ control, ${ }^{* *}$ indicates a significant increase from the 1.1 $\mathrm{Pa}$ group generated by the same perfusate. $\dagger$ indicates a significant decrease from the RBC perfusion group at the same shear stress magnitude. 
Figure 6

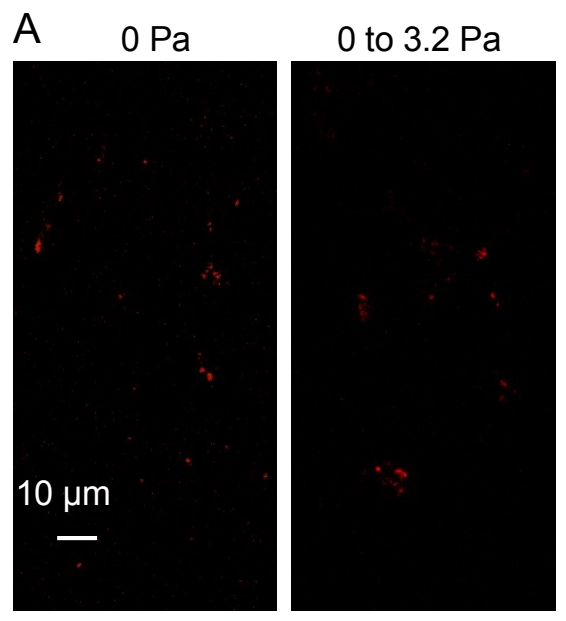

Plasma

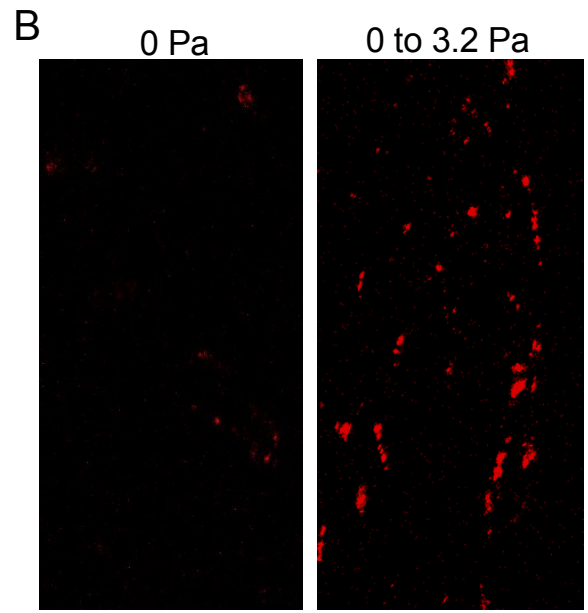

Whole Blood

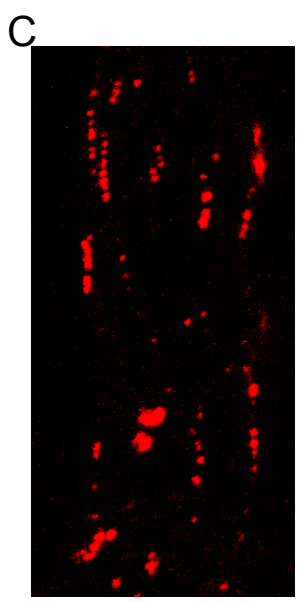

0 to $3.2 \mathrm{~Pa}$

$40 \% \mathrm{RBC}$ in $1 \%$ albumin-Ringer
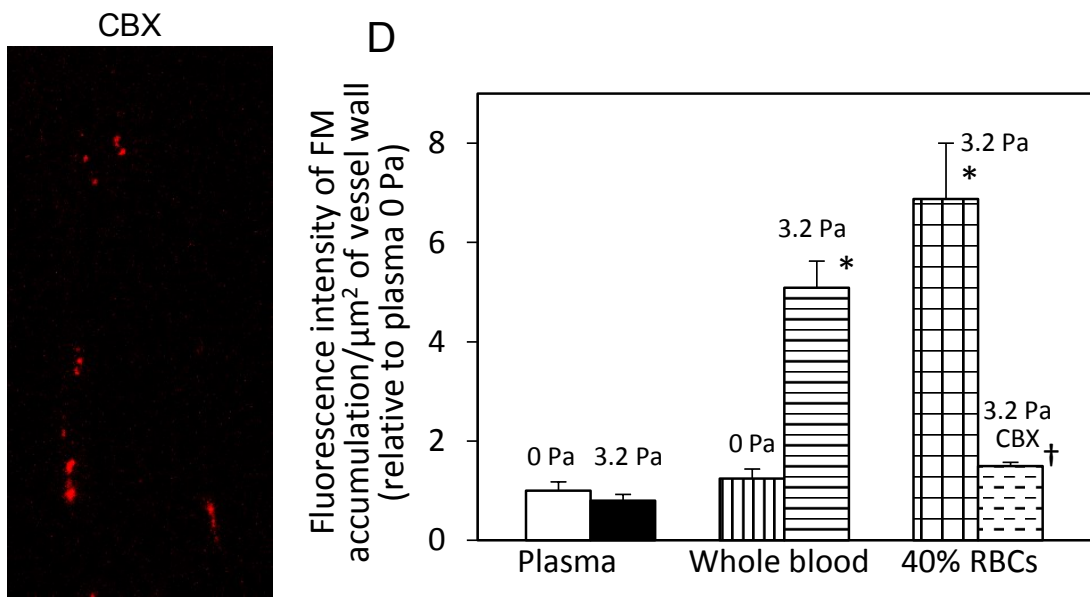
Figure 6. Blood flow and RBC solution perfusion generated-sudden change of shear stress induced endothelial cell gap formation. A) Representative confocal images shows blood but not plasma perfusion generated sudden change of shear stress from 0 to 3.2 $\mathrm{Pa}$ induced gap formations B) Representative confocal images shows $40 \% \mathrm{RBC}$ solution generated sudden change of shear stress induced gap formations. While preincubation of RBC solution with cabenoxolone abolished the gap formation. C) Quantificantion of the fluorescence intensity $(\mathrm{FI})$ of accumulated fluorescent microspheres (FMs) accumulation at endothelial junction. ( $\mathrm{n}=3$ for Plasma $0 \mathrm{~Pa}$, Plasma 3.2 $\mathrm{Pa}$ and Blood $0 \mathrm{~Pa}, \mathrm{n}=6$ for Blood 3.2 $\mathrm{Pa}$ and $40 \% \mathrm{RBC}+\mathrm{CBX}, \mathrm{n}=5$ for $40 \% \mathrm{RBC} 3.2 \mathrm{~Pa}$ ) * indicates a significant increase from the $0 \mathrm{~Pa}$ control, $\uparrow$ indicates significant decreases from the RBC perfusion group. 
Figure 7.

A
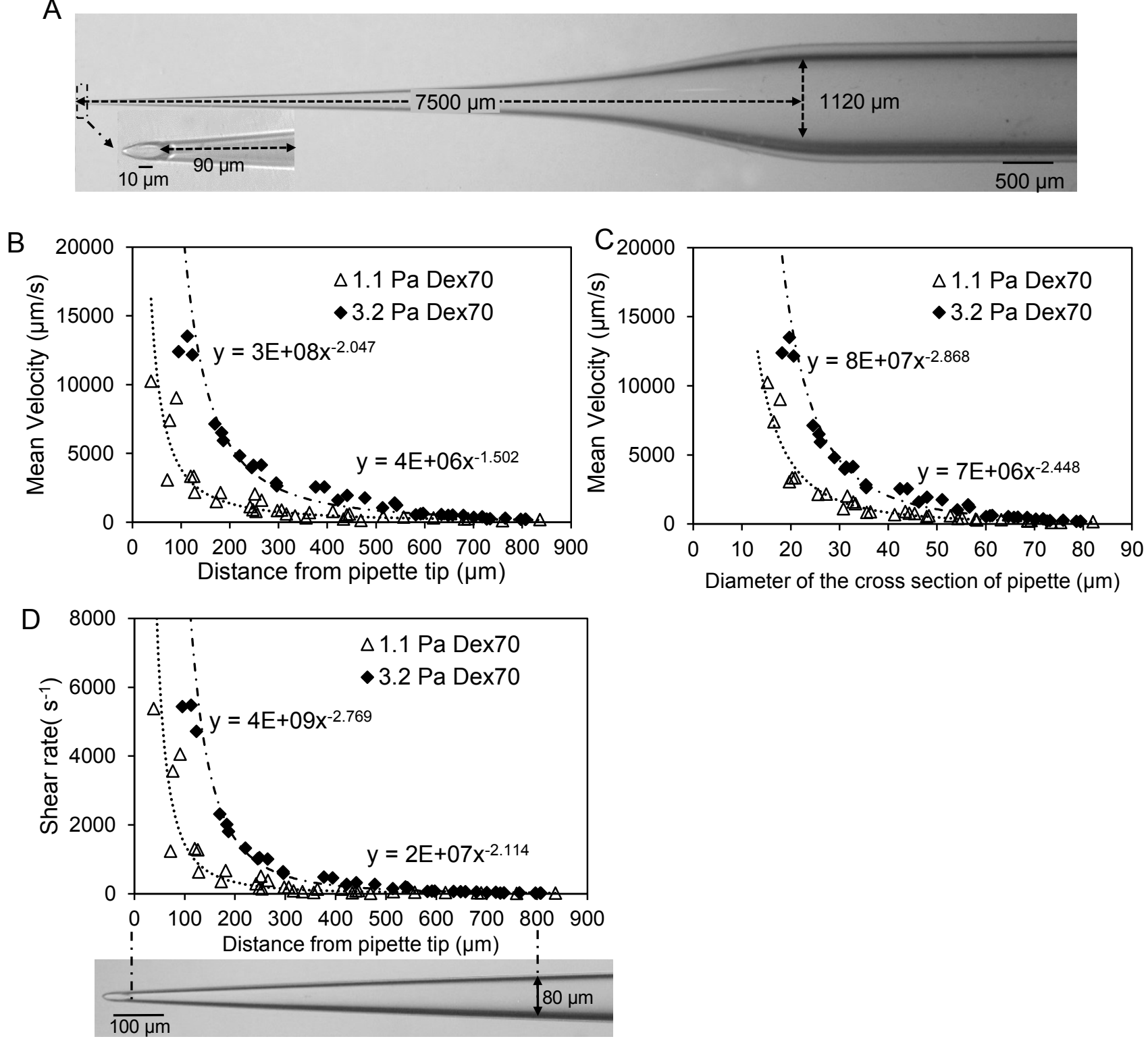
Figure 7. Fluid velocity measurement inside cannulation pipette. A) A representative image shows the dimension of a cannulation pipette. The changes in the pipette inner diameter are relatively linear within $800 \mu \mathrm{m}$ distance from the cannulation pipette tip where fluid velocity was measured. B-D) Flow velocity and shear rate along the cannulation pipette when the vessel lumen wall shear stress was suddenly changed from 0 to 1.1 and 3.2 $\mathrm{Pa}$, respectively. 


\title{
CHAPTER 4
}

THE LOSS OF GLYCOCALYX IN DIABETIC MICROVESSELS CONTRIBUTES TO THE EXCESSIVE SHEAR STRESS-INDUCED ENDOTHELIAL NITRIC OXIDE PROCUTION

\author{
Sulei Xu, Dong Yuan and Pingnian He \\ Department of Physiology and Pharmacology, School of medicine, West Virginia \\ University, Morgantown, WV 26506
}

Running Title: Reduced NO by applied caveolin-1 promotes leukocyte adhesion

Address for proofs:

Dr. Pingnian $\mathrm{He}$

Department of Physiology and Pharmacology

School of Medicine

West Virginia University

Morgantown, WV 26506-9229

Tel: (304) 293-1515

Fax: (304) 293-3850

E-Mail: phe@hsc.wvu.edu 


\begin{abstract}
Glycocalyx (GCX), a layer of proteoglycans covering the endothelium, has been implicated as a mechanical sensor in sensing the changes in shear stress (SS) in vitro. Though acute hyperglycemia has been reported to impair GCX in human by indirect volume estimation, the direct experimental evidence and how the impaired GCX affects SS-mediated endothelial function in vivo remain to be demonstrated. The objective of this study is to identify the changes in GCX in microvessels of streptozotocin-induced diabetic rats and evaluate the associated changes in sensing SS and SS-induced nitric oxide (NO) production in individually perfused venules of diabetic rats. GCX is visualized either by electron microscope (EM) or by lectin fluorescence labeling. EC NO production was quantified in DAF-2 DA loaded vessels using fluorescence imaging. Microvessels of diabetic rats showed degraded GCX by both EM and confocal images, a similar observation found in pronase $(0.1 \mathrm{mg} / \mathrm{ml}, 3 \mathrm{~min})$ treated microvessels. Unexpectedly, the EC NO production in response to blood flow-generated changes in SS in diabetic vessels was significantly higher than that in normal vessels. The NO production rate in normal vessels were $0.13 \pm 0.02$ and $0.24 \pm 0.02 \mathrm{AU} / \mathrm{min}$ at 11 and $32 \mathrm{dyn} / \mathrm{cm}^{2}$. In contrast, the NO production rate of diabetic vessel in response to the same magnitude changes of SS were $0.25 \pm 0.05$ and $0.37 \pm 0.03 \mathrm{AU} / \mathrm{min}$, respectively. These findings were also supported by an increased NO production observed in pronase treated normal venules. Our results indicate that the impaired GCX in diabetic microvessels enhances EC response to mechanical force and potentiates NO production.
\end{abstract}

Key Words: glycocalyx, diabetic, microvessel permeability, nitric oxide 


\section{INTRODUCTION}

Wall shear stress is the tangential force exerted per unit area of the blood-endothelium surface. How the endothelium senses changes in shear stress in intact microvessels has not been well explored. The glycocalyx has been implicated as a mechanical sensor for changes in shear stress. Using an indirect volume estimation method, endotoxemia and hyperglycemia have been reported to cause damage to the glycocalyx coating on the endothelial cell surface in human. However, direct experimental evidence and how the impaired glycocalyx (GCX) affects shear stress-mediated endothelial function in vivo remain to be demonstrated. A study in frog microvessels showed increases in hydraulic conductivity (Lp) to be associated with the partial digestion of the endothelial glycocalyx. Our recent study found increased basal permeability and enhanced permeability responses to inflammatory mediators in venules of diabetic rats. Whether the increased permeability in diabetic rats is the result of an impaired glycocalyx remains unknown. Here we conducted experiments to test the hypothesis that hyperglycemia-induced damages to the glycocalyx contribute to the increases in microvessel permeability in diabetic rats. Among the currently reported vasoactive factors induced by shear stress, NO released from ECs is considered as the most important factor in the regulation of vascular function. In the arterioles, it regulates vascular tone, peripheral resistance, and tissue perfusion. In venular microvessels, as demonstrated in previous studies from our lab and others, basal NO plays anti-inflammatory roles by preventing leukocyte adhesion and platelet aggregation, whereas stimuli-induced excessive NO production plays a pro-inflammatory role and increases microvessel permeability. Therefore, this study was focused on shear stressinduced NO production under normal and diabetic conditions. 


\section{METHODS}

\section{Animal preparation}

Experiments were carried out on venular microvessels in rat mesenteries. All procedures and animal use were approved by the Animal Care and Use Committee at West Virginia University. Female Sprague-Dawley rats (2-3-mo old; body wt 220-250 g; Hilltop Laboratory Animals; Scottdale, PA) were anesthetized with pentobarbital sodium given subcutaneously. The initial dosage was $65 \mathrm{mg} / \mathrm{kg}$ of body weight with an additional $3 \mathrm{mg}$ dose given as needed. The trachea was intubated, and a midline surgical incision (1.5-2 $\mathrm{cm}$ ) was made in the abdominal wall. The mesentery was gently moved out of the abdominal cavity and spread over a glass cover slip attached to an animal tray. The upper surface of the mesentery was continuously superfused with mammalian Ringer solution. The temperature of the superfusate was maintained at $37^{\circ} \mathrm{C}$. All experiments were carried out on venular microvessels with diameters ranging between 40 and $50 \mu \mathrm{m}$. Each experiment was performed on a single microvessel with one experiment per animal.

\section{Fluorescence imaging of endothelial nitric oxide production}

Endothelial NO production was measured in DAF-2 DA loaded vessels using fluorescence imaging. Experiments were performed on a Nikon Diaphod 300 microscope equipped with a 12-bit digital CCD camera (ORCA; Hamamatsu) and a computer controlled shutter (Lambda 10-2; Sutter Instrument; Novato, CA). A 75-W xenon lamp was used as the light source. The excitation wavelength for DAF-2 was selected by an interference filter (480/40 $\mathrm{nm})$, and emission was separated by a dichroic mirror $(505 \mathrm{~nm})$ and a band-pass barrier $(535 / 50 \mathrm{nM})$. All the images were acquired and analyzed using Metafluor software (Universal Imaging, West Chester, PA). To minimize the photo-bleaching, a neutral density 
filter (0.5 ND) was positioned in front of the interference filter and the exposure time was minimized to $0.12 \mathrm{sec}$ at $1 \mathrm{~min}$ intervals. During the experiments, each vessel was first perfused with albumin-Ringer solution containing DAF-2 DA (5 $\mu \mathrm{M})$. Image collection was started after 40 minutes of DAF-2 DA perfusion. All images were collected from a group of endothelial cells located in the same focal plane of the vessel wall using a Nikon Fluor lens (x20, numerical aperture, 0.75). Quantitative analysis was conducted at the individual endothelial cell level using manually selected regions of interest (ROls) along the vessel wall. Each ROI covers the area of one individual cell as indicated by the fluorescence outline. The tissue autofluorescence was subtracted from all of the measured Fls. Shear stress-induced changes in fluorescence intensity $(\mathrm{FI})$ were expressed as $\left(\mathrm{FI} / \mathrm{FI} \mathrm{l}_{0}\right) \mathrm{X} 100$, where $\mathrm{Fl}_{0}$ is control $\mathrm{Fl}$ level. The NO production rate was calculated as $\mathrm{dFI} / \mathrm{dt}$. Details have been described previously. ${ }^{1}$

\section{Electron microscopy}

The changes of glycocalxy was examined with electron microscopy. Each vessel was perfused with Alcian Blue $8 \mathrm{GX}(0.05 \%)$ to stain the glycocalyx, while the upper surface of the mesentery was superfused with a fixative solution. After fixation, a small panel of the mesentery that contained the perfused microvessel was dissected and placed in the same fixative at $4^{\circ} \mathrm{C}$ overnight. The specimens were then rinsed in $0.1 \mathrm{M}$ phosphate buffer and permeabilized with $50 \%$ ethanol. The samples were postfixed and transferred into propylene oxide, and embedded in epon. Photomicrographs were taken on a JEOL 1220 transmission electron microscope

\section{Confocal imaging}


Glycocalyx distrubution was also visualized with confocal imaging using FITC-conjugatedlectin (FITC-BS-1) that can specifically bind to components of the endothelial glycocalyx. Leica TCS SL confocal microscope equipped with microvessel perfusion rig was used to collect images. Each stack of images was acquired by optical sectioning at successive XY focal planes with vertical depth of $0.5 \mu \mathrm{m}$ using Leica X 25 objective (HC Plan APO, NA 0.95 ) and $1024 \times 1024$ scanning format. Leica software was used for image acquisition and image analysis.

\section{Visualization of Endothelial gaps formation in intact microvessels}

We used fluorescent microspheres as the marker to identify endothelial gap formation. Briefly, each individual microvessel was perfused with a perfusate containing red fluorescent microspheres (FMs, $100 \mathrm{~nm}, 3.6 \times 10^{11} / \mathrm{ml}$ ) with and without the sudden change of shear stress for $10 \mathrm{~min}$. Confocal images were collected following $10 \mathrm{~min}$ albumin-Ringer perfusion to remove the free FMs from the vessel lumen. A stack of confocal images was obtained from each vessel at successive $X-Y$ focal planes with vertical depth of $0.5 \mu \mathrm{m}$ using Leica $\times 25$ (NA 0.95) objective. The accumulation of FMs at junctions between endothelial cells was analyzed in images from lower half of the complete Z-axis image stack of the vessel. The total FI of FM (area $x$ depth $x$ mean intensity/pixel) for each vessel segment was quantified as total intensity/surface area of the vessel wall under control or shear conditions. Details have been described previously. ${ }^{2}$

\section{Solutions and reagents}

Mammalian Ringer's solution was used for the experiments. The composition of the mammalian Ringer's solution was (mM) $132 \mathrm{NaCl}, 4.6 \mathrm{KCl}, 2 \mathrm{CaCl}_{2}, 1.2 \mathrm{MgSO}_{4}, 5.5$ 
glucose, $5.0 \mathrm{NaHCO}_{3}, 20 \mathrm{~N}-2$ hydroxyethylpiperazine-N'-2-ethanesulfonic acid (HEPES) and Na-HEPES pH 7.4. Albumin-Ringer's solution (BSA, $10 \mathrm{mg} / \mathrm{ml}$ ) was used as control solution. Fura-2 AM was purchased from Life Technologies, Grand Island, NY. DAF-2 DA was purchased from Sigma, St. Louis, MO. All the fluorescent dyes were prepared in DMSO for stock solution and at least 1:1000 dilution was made for the final working solutions. All of the perfusates containing the test reagents were freshly prepared before each cannulation.

\section{Data analysis and statistics}

All values are mean \pm SE. Paired t-test was used for paired data analysis. ANOVA was used to compare data between groups. A probability value of $\mathrm{P}<0.05$ was considered statistically significant. 


\section{RESULTS}

\section{Impaired glycocalyx in diabetic rat venules}

Cultured cell studies showed that glycocalyx degradation is correlated with decreases in shear stress-induced NO production and with increases in permeability of EC monolayers. ${ }^{3-5}$ Decreased glycocalyx volume has been reported in diabetic patients. ${ }^{6} \mathrm{~A}$ study in frog microvessels showed increases in hydraulic conductivity (Lp) to correlate with the partial digestion of the endothelial glycocalyx. ${ }^{7}$ However, whether the hyperpermeability in diabetic rats is the result of impaired glycocalyx has not been studied in vivo. We hypothesize that the hyperglycemia caused glycocalyx damage may contribute to the increases in microvessel permeability in our STZ-induced diabetic rats. The electron micrograph with Alcian Blue $8 \mathrm{GX}$ staining shown in Fig $1 \mathrm{~A}$ directly shows that the glycocalyx was impaired in microvessels of diabetic rats. In addition to the electron microscopic studies, we developed another method to visualize the glycocalyx distribution using confocal imaging with FITC-conjugated-lectin (FITC-BS-1) that can specifically bind to components of the endothelial glycocalyx. ${ }^{8}$ Fig $1 \mathrm{~B}$ lower left panel is a single scan of the FITC-BS-1 labeled glycocalyx of a normal vessel (focal plane at the center of the vessel lumen) and Fig 1B upper panel is the projection image from the lower half $X-Y$ z-stack images. Fig 1B also shows the disappearance of glycocalyx after 5 min of pronase $(0.1 \mathrm{mg} / \mathrm{ml})$ treatment.

\section{Impaired glycocalyx enhances EC NO production to shear stress}

The glycocalyx has been indicated as a mechanotransduction sensor to the fluid shear stress in many in vitro studies. ${ }^{9-12}$ Using enzyme degradation of its specific components 
impaired the shear stress induced NO production and increased permeability in vitro. ${ }^{13}$ In the proposed studies, we will investigate the role of the glycocalyx in the regulation of shear stress-induced changes in microvessel functions in intact venules. Since NO is one of most importance EC vascular factor induced by shear stress, we first investigate the change of EC NO to shear stress in diabetic rats with impaired glycocalyx. Our results in Fig 2 showed enhanced NO production when normal blood was perfused into diabetic venules. The NO responses to different magnitudes of shear stress were even higher than those measured in pronase treated normal venules. These results suggested that both a damaged glycocalyx and altered ECs may contribute to this enhanced EC NO production to shear stress.

\section{Increased microvessel permeability to shear stress in diabetic rats}

Excessive NO production induced by inflammatory mediators contributes to an increase in microvessel permeability and plays a pro-inflammatory role. ${ }^{14-16}$ Thus, our next step is to investigate whether the microvessel permeability changes to shear stress in enhanced in diabetic rats. Our previous study found that the changes in the magnitude of FM accumulation closely correlated with the time course of PAF-induced increases in hydraulic conductivity $(L(p))$, indicating that the accumulation of FM reflects the degree of EC gaps formation and related microvessel permeability. ${ }^{2}$ In Fig 3, venules of diabetic rats perfused with $1 \% \mathrm{BSA}$ (control) at $1.1 \mathrm{~Pa}$ shows moderate gap formation. Blood perfusion-generated shear stress at 3.2 Pa significantly potentiated the EC gaps formation (compare the right panels between $A$ and $B$ ), indicating increased sensitivity of diabetic vessels in response to mechanical force. 


\section{DISCUSSION}

Wall shear stress is the tangential force exerted per unit area of the blood-endothelium surface. The glycocalyx, a layer of proteoglycans covering the endothelium, has been indicated to be a mechanotransduction sensor for changes of shear stress. ${ }^{12}$ Impaired glycocalyx has been suggested may directly contribute to the increased leukocyte adhesion, hyperpermeability, and other microvascular dysfunctions associated with diabetes and other cardiovascular diseases. In our present study, we found the impaired glycocalyx increases the endothelial susceptibility to shear stress, which resulting in augmented nitric oxide production in microvessels of diabetic rats.

The experiments were conducted in individually perfused microvessels with undisturbed surrounding circulation, which we consider extremely important. In order to translate the remarkable knowledge gained from in vitro studies into clinical therapeutic applications, it is essential to evaluate cellular and molecular mechanisms in their native state. Multidimensional protein identification revealed that $41 \%$ of proteins expressed in vivo are not detected in vitro, indicating that distinct in vivo protein expression is apparently regulated by the tissue microenvironment and cannot yet be duplicated in standard cell culture. ${ }^{17}$ This is specifically important for glycocalyx studies, because the glycocalyx under static cultured cell growth is not as well developed as that under physiological conditions.

The endothelial cell glycocalyx has been reported to have 0.2 to $0.5 \mu \mathrm{m}$ thickness lining the luminal surface of all blood vessels. ${ }^{18} \mathrm{~A}$ human study indicated that glycocalyx volume is reduced in hyperglycemia patients. ${ }^{6}$ However, they estimated systemic glycocalyx volume by comparing the distribution of glycocalyx permeable tracer (dextran 40) with glycocalyx impermeable tracer (using labeled erythrocytes). This methodology raises some 
concerns for its validity. ${ }^{19}$ No direct evidence had previously been demonstrated for an impaired glycocalyx in diabetes. Here, we directly visualize the loss of glycocalyx in diabetic animal using electron microscopy and immunofluorescence confocal imaging.

The glycocalyx has been indicated as a mechanotransduction sensor to the fluid shear stress in many in vitro studies. ${ }^{9-12}$ Using enzyme degradation of its specific components impaired the shear stress induced NO production and increased permeability in vitro. ${ }^{13}$ Our data suggested that removing the glycocalyx enhanced EC NO production, which indicated that impaired glycocalyx enhances EC responses to mechanical force. Interestingly, we also found the NO responses to different magnitudes of shear stress were even higher than those measured in pronase treated normal venules. It suggested that besides the damaged glycocalyx, the altered ECs in diabetic rats may also contribute to this enhanced EC NO production to shear stress.

Nitric oxide (NO) is mainly produced from endothelial cells driving from shear stress in the vasculature under physiological condition. It has been currently demonstrated to play important roles in the regulation of microvessel barrier function. Basal level of endothelial NO production protects vascular wall from leukocytes adhesion, platelets aggregation, thrombosis, and atherosclerotic plaque formation. ${ }^{20}$ On the other hand, inflammatory mediator-stimulated increased NO production in endothelial cells is essential for increasing the microvessel permeability. ${ }^{21,22}$ Our previous study on individually perfused intact venules showed that platelet activating factor (PAF) induces transient increases in microvessel permeability through both $\mathrm{Ca}^{2+}$ and $\mathrm{NO}$ dependent pathway. ${ }^{23}$ However, the exactly role of $\mathrm{NO}$ in regulating microvessel permeability is still unknown. 
Our future study will further investigate the causal relationship among impaired glycocalyx, enhanced shear stress induced NO production, and enhanced vascular permeability, as well as the involved cellular mechanisms.

\section{GRANT SUPPORT}

This study was supported by National Heart, Lung, and Blood Institute grants HL56237

and HL084338 (He, P.) and American Heart Association Great Rivers Affiliate 12PRE11470010 pre-doctoral fellowship (Xu, S.). 


\section{REFERENCE}

1. Zhu L, He P. Platelet-activating factor increases endothelial $[\mathrm{Ca} 2+] \mathrm{i}$ and $\mathrm{NO}$ production in individually perfused intact microvessels. Am J Physiol Heart Circ Physiol. 2005;288(6):H2869-2877.

2. Jiang $Y$, Wen $K$, Zhou $X$, Schwegler-Berry D, Castranova V, He P. Threedimensional localization and quantification of PAF-induced gap formation in intact venular microvessels. Am J Physiol Heart Circ Physiol. 2008;295(2):H898-906.

3. Florian JA, Kosky JR, Ainslie K, Pang Z, Dull RO, Tarbell JM. Heparan sulfate proteoglycan is a mechanosensor on endothelial cells. Circ Res. 2003;93(10):e136142.

4. Pahakis MY, Kosky JR, Dull RO, Tarbell JM. The role of endothelial glycocalyx components in mechanotransduction of fluid shear stress. Biochem Biophys Res Commun. 2007;355(1):228-233.

5. Lopez-Quintero SV, Amaya R, Pahakis M, Tarbell JM. The endothelial glycocalyx mediates shear-induced changes in hydraulic conductivity. Am J Physiol Heart Circ Physiol. 2009;296(5):H1451-1456.

6. Nieuwdorp M, van Haeften TW, Gouverneur MC, Mooij HL, van Lieshout MH, Levi M, Meijers JC, Holleman F, Hoekstra JB, Vink H, Kastelein JJ, Stroes ES. Loss of endothelial glycocalyx during acute hyperglycemia coincides with endothelial dysfunction and coagulation activation in vivo. Diabetes. 2006;55(2):480-486.

7. Adamson RH. Permeability of frog mesenteric capillaries after partial pronase digestion of the endothelial glycocalyx. J Physiol. 1990;428:1-13.

8. Mulivor AW, Lipowsky HH. Inflammation- and ischemia-induced shedding of venular glycocalyx. Am J Physiol Heart Circ Physiol. 2004;286(5):H1672-1680.

9. Mulivor AW, Lipowsky HH. Role of glycocalyx in leukocyte-endothelial cell adhesion. Am J Physiol Heart Circ Physiol. 2002;283(4):H1282-1291.

10. Reitsma S, Slaaf DW, Vink H, van Zandvoort MA, oude Egbrink MG. The endothelial glycocalyx: composition, functions, and visualization. Pflugers Arch. 2007;454(3):345-359.

11. Weinbaum S, Tarbell JM, Damiano ER. The structure and function of the endothelial glycocalyx layer. Annu Rev Biomed Eng. 2007;9:121-167.

12. Weinbaum S, Zhang $X$, Han $Y$, Vink H, Cowin SC. Mechanotransduction and flow across the endothelial glycocalyx. Proc Natl Acad Sci U S A. 2003;100(13):79887995. 
13. Mochizuki S, Vink H, Hiramatsu O, Kajita T, Shigeto F, Spaan JA, Kajiya F. Role of hyaluronic acid glycosaminoglycans in shear-induced endothelium-derived nitric oxide release. Am J Physiol Heart Circ Physiol. 2003;285(2):H722-726.

14. He P, Liu B, Curry FE. Effect of nitric oxide synthase inhibitors on endothelial [Ca2+]i and microvessel permeability. Am J Physiol. 1997;272(1 Pt 2):H176-185.

15. Wu HM, Huang Q, Yuan Y, Granger HJ. VEGF induces NO-dependent hyperpermeability in coronary venules. Am J Physiol. 1996;271(6 Pt 2):H2735-2739.

16. Mayhan WG. Nitric oxide accounts for histamine-induced increases in macromolecular extravasation. Am J Physiol. 1994;266(6 Pt 2):H2369-2373.

17. Durr E, Yu J, Krasinska KM, Carver LA, Yates JR, Testa JE, Oh P, Schnitzer JE. Direct proteomic mapping of the lung microvascular endothelial cell surface in vivo and in cell culture. Nat Biotechnol. 2004;22(8):985-992.

18. Zuurbier CJ, Demirci C, Koeman A, Vink H, Ince C. Short-term hyperglycemia increases endothelial glycocalyx permeability and acutely decreases lineal density of capillaries with flowing red blood cells. J Appl Physiol. 2005;99(4):1471-1476.

19. Michel CC, Curry FR. Glycocalyx volume: a critical review of tracer dilution methods for its measurement. Microcirculation. 2009;16(3):213-219.

20. Albrecht EW, Stegeman CA, Heeringa $\mathrm{P}$, Henning $\mathrm{RH}$, van Goor $\mathrm{H}$. Protective role of endothelial nitric oxide synthase. J Pathol. 2003;199(1):8-17.

21. Brkovic A, Sirois MG. Vascular permeability induced by VEGF family members in vivo: role of endogenous PAF and NO synthesis. J Cell Biochem. 2007;100(3):727737.

22. Bucci M, Gratton JP, Rudic RD, Acevedo L, Roviezzo F, Cirino G, Sessa WC. In vivo delivery of the caveolin-1 scaffolding domain inhibits nitric oxide synthesis and reduces inflammation. Nat Med. 2000;6(12):1362-1367.

23. Pagakis SN, Curry FE. Imaging of $\mathrm{Ca} 2+$ transients in endothelial cells of single perfused capillaries: correlation of peak $[\mathrm{Ca} 2+] \mathrm{i}$ with sites of macromolecule leakage. Microcirculation. 1994;1(4):213-230. 


\section{FIGURES AND FIGURE LEGENDS}

Figure 1.

A
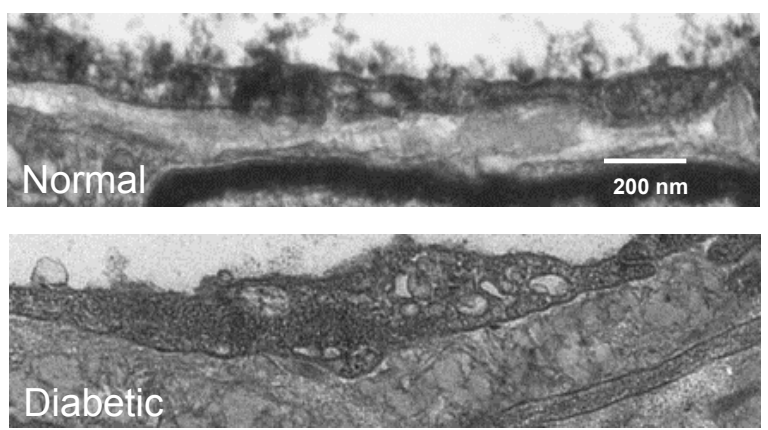

B

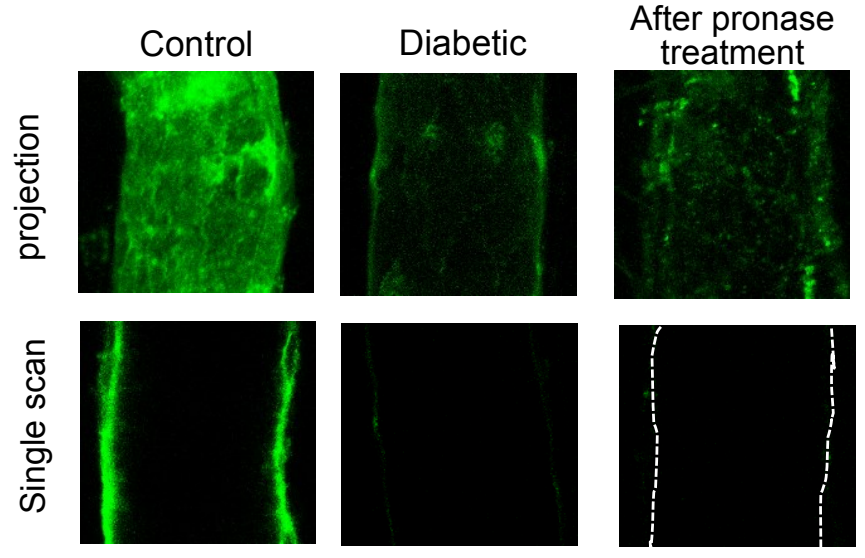

Figure 1 Direct visualization of glycocalyx in rat venules using EM and confocal imaging A: The electron micrograph with Alcian Blue 8GX staining of GCX shows impaired GCX in a rat mesenteric venule. B: Confocal images illustrating glycocalyx by FITCconjugated-lectin (FITC-BS-1) staining in intact mesenteric venules. Upper panel is the projection image from the lower half $X-Y$ Z-stack images. Lower panel is a single scan of the FITC-BS-1 labeled glycocalyx of a normal vessel (focal plane at the center of the vessel lumen). Fluorescence intensity is significantly decreased in diabetic and pronase $(0.1 \mathrm{mg} / \mathrm{ml}, 5 \mathrm{~min})$ treated venules, indicating the loss of glycocalyx layer at endothelial surface. 
Figure 2

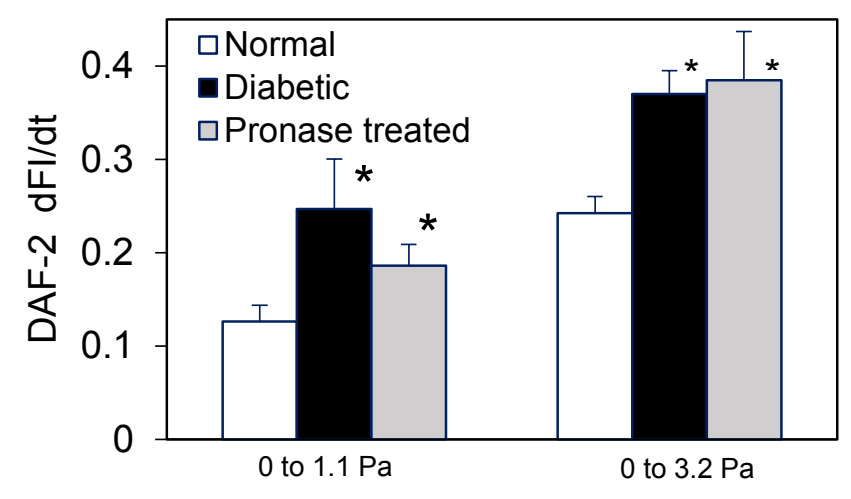

Figure 2. Endothelial NO production in response to blood flow-generated shear stress is significantly enhanced in both diabetic and pronase treated rat mesenteric venules. Shear stress was changed from 0 to $1.1 \mathrm{~Pa}$ or $3.2 \mathrm{~Pa}$, in each experiment, respectively. The graph shows that the NO production rates in diabetic and pronase treated vessels were significantly higher than that in a normal vessel in response to the same range of changes in shear stress, indicating the impaired glycocalyx increased endothelial sensitivity to mechanical force ( $\mathrm{N}=3$ in each group). 


\section{Figure 3}

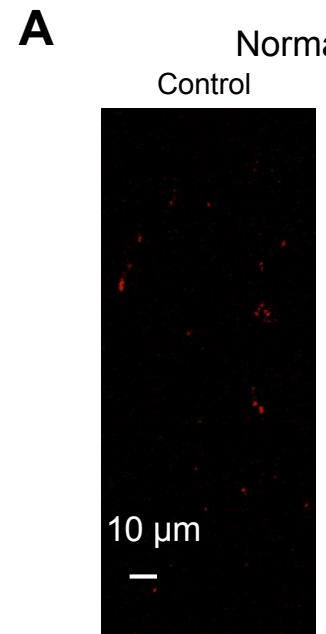

1.1 $\mathrm{Pa}$

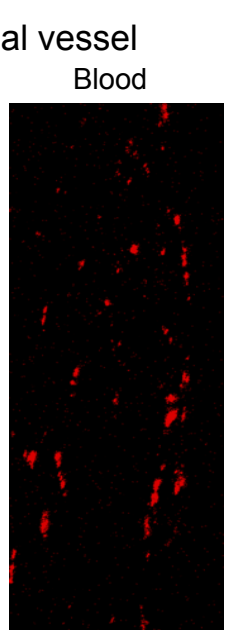

3.2 $\mathrm{Pa}$

B

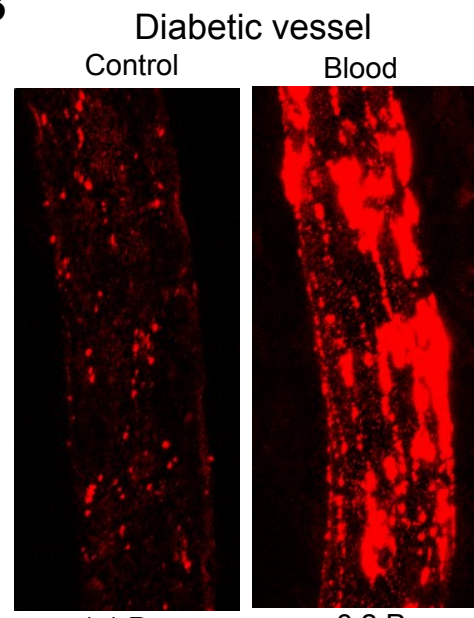

1.1 Pa

3.2 Pa

\section{Figure 3. Shear stress-induced EC gap formation is enhanced in venules of diabetic}

rats. Accumulation of fluorescent microspheres (FMs, red) is used to mark endothelial gaps. Images were collected after the start of changing shear stress with different perfusate. Each image is the projection of the lower half of the z-stack images. A) Venules of normal rats perfused with $1 \% \mathrm{BSA}$ (control) at $1.1 \mathrm{~Pa}$ shows no FM accumulation and perfusion of blood at 3.2 Pa induces small magnitude of EC gaps; B) Venules of diabetic rats perfused with $1 \% \mathrm{BSA}$ (control) at $1.1 \mathrm{~Pa}$ shows moderate gap formation, which may represent the moderate basal $L p$ increase found in diabetic rats and can be independent from the shear stress (left panel). A sudden change of blood perfusion-generated shear stress to $3.2 \mathrm{~Pa}$ significantly potentiated the EC gaps formation (compare the right panels between $A$ and $B$ ), indicating increased sensitivity of diabetic vessels in response to mechanical force. 


\title{
CHAPTER 5
}

\section{IN VITRO RECAPITULATION OF FUNCTIONAL MICROVESSELS FOR THE STUDY OF ENDOTHELIAL SHEAR RESPONSE, NITRIC OXIDE AND [Ca $\left.{ }^{2+}\right]$}

\author{
Xiang $\mathrm{Li}^{1 \neq}$, Sulei $\mathrm{Xu}^{2 \neq}$, Pingnian $\mathrm{He}^{2}$, Yuxin Liu ${ }^{1,{ }^{*}}$ \\ ${ }^{1}$ Lane Department of Computer Science and Electrical Engineering \\ West Virginia University, Morgantown, WV 26506 \\ ${ }^{2}$ Department of Physiology and Pharmacology \\ West Virginia University, Morgantown, WV 26506 \\ * Corresponding Author: Tel: 3042939144; Fax: 3042938602; e-mail: \\ yuxin.liu@mail.wvu.edu \\ \# Equal contribution authors
}




\begin{abstract}
A physiologically realistic microvessel model for studying complex vascular phenomena, such as endothelial cell signaling and barrier functions, is much needed to biologists because of critical constraints of current microvessel models having the resolution at individual cell levels, in vitro models are lack of stable and mature endothelium with appropriate barrier functions, while in vivo animal studies are often too complex and have high experimental costs. Microfluidic technologies have enabled in vitro studies to closely simulate the in vivo microvessel environment with sufficient complexity. However, there are still gaps of knowledge and a generalized lack of validation of these models as adequate model of a functional microvessel. In this paper, we presented an engineered microfluidic microvessel model, demonstrated in vitro formation of microvessels that recapitulate key features of the microvessels in vivo, and validated its utility for biological applications. Our microvessel model illustrates unique capabilities in advanced micromanufacturing and microfluidic technologies to create a model mimicking the dimensions of in vivo microvessels with long-term, continuous perfusion control, as well as real-time high-quality imaging capability. Cells in our model maintain their phenotype, viability, proliferation with proper barrier functions, and respond to flow shear force and inflammatory stimuli. In particular, primary human umbilical vein endothelial cells were successfully cultured along the entire inner surface of the microchannel network with morphologies close to those reported in venular vasculature and well-developed VE-cadherin junctions throughout the channels. The endothelial cell responses to shear stresses were quantified under different flow shear conditions. Furthermore, we successfully measured agonist-induced changes in intracellular $\mathrm{Ca}^{2+}$ concentration $\left[\mathrm{Ca}^{2+}\right]$ i and nitric oxide (NO) production in individual
\end{abstract}


endothelial cell levels using our model. The results were similar and comparable to those derived from individually perfused intact venules. With the in vivo validation of its functionalities, our microfluidic model demonstrates a great potential for biological applications and bridging the gaps between in vitro and in vivo microvascular research.

Key words: microfluidics, HUVECs, microvessel network, shear responses, ATP, $\left[\mathrm{Ca}^{2+}\right]$, nitric oxide 


\section{INTRODUCTION}

The development of microfluidic devices has been embraced by engineers over two decades. However, the adaptation and application of microfluidics in mainstream biology is still lacking. According to the recent summary, the majority publications of microfluidics are still in engineering journals (85\%). ${ }^{1}$ The equivalent or improved performance of microfluidic devices is not convincing enough for many biologists to switch from current state-of-the-art techniques such as Transwell assay and macroscale culture dish/glass slide. ${ }^{1,2}$ The most common way to replicate certain features of in vivo shear stress by biologist includes parallel flow chamber and cone and plate viscometer. ${ }^{3}$ Microfluidics has the advantage to provide a solution that has not been addressed by those macroscale approaches, such as developing a more physiologically relevant in vitro model. In this paper we continue our previous efforts in developing an in vitro functional microvessels that could provide a platform for the study of complex vascular phenomena. ${ }^{4}$

A physiologically realistic microvessel model is able to overcome certain critical constraints of the current in vitro models, such as creating a stable and mature endothelium with appropriate barrier functions. Additionally, the advanced models, that enable a close simulation of in vivo environment with sufficient complexity, could work as artificial surrogates for curtailing high experimental costs and complexities associated with animals and in vivo studies. ${ }^{5}$ Several groups have pioneered in the development of advanced microvessel models, which applied micromanufacturing and microfluidic techniques, used either polymer or hydrogel to template the growth of vascular endothelial cells (ECs), cocultured ECs with other vascular cells, demonstrated cell viability, and appropriate functions in angiogenesis and thrombosis. ${ }^{6-9}$ Nonetheless, prior methodologies did not demonstrate 
and quantify some key features for microvessels, such as intracellular $\mathrm{Ca}^{2+}$ concentration $\left(\left[\mathrm{Ca}^{2+}\right]_{i}\right)$ and nitric oxide (NO) production in cultured microvessels with well-developed endothelial junctions. NO, is essential for controlling vascular tone and resistance in arterioles, and regulating vascular wall adhesiveness and permeability in venules. ${ }^{10}$ Additionally, the endothelial $\left[\mathrm{Ca}^{2+}\right]_{i}$ plays an important role in microvessel permeability, ${ }^{11,12}$ angiogenesis ${ }^{13}$ and morphogenesis. ${ }^{14}$ However, in microfluidic based systems, there are very limited reports for measurements of $\left[\mathrm{Ca}^{2+}\right]$ and NO production. ${ }^{15,16,19,20}$ Some studies reported DAF-2DA loaded endothelial cells from a microfluidic network, ${ }^{17,18}$ and ATPinduced NO production was reported on cultured bovine pulmonary artery endothelial cells within a straight microfluidic microchannel. However, there is a lack of reports of quantification of time-dependent changes in NO production under basal and stimulated conditions, and agonist-induced changes in $\left[\mathrm{Ca}^{2+}\right]_{i}$ in endothelialized microchannels.

In this paper, we presented an in vitro formation of a microvessel network that recapitulates key features of microvessels in vivo. The microfabrication technique accurately controls the dimensions of the developed microchannels in microscales which mimicking those of in vivo microvessels. In addition, continuous microfluidic perfusion is able to control the mass transfer and flow shear stresses precisely. A confluent endothelial monolayer was formed and fully covered inside the entire microchannel network. To further validate the functionalities of developed microvessels, the vascular endothelial adherens junctions were confirmed by the VE-cadherin immunofluorescence staining. We also evaluated the cell morphology changes in response to different patterns of shear stress. Additionally, endothelial $\left[\mathrm{Ca}^{2+}\right]$ and $\mathrm{NO}$ production were quantitatively measured under control and stimulated conditions. 


\section{MATERIAL AND METHODS}

\section{Design and Fabrication}

The microchannel network designed in this paper was a three-level branching microchannels as shown in Fig. 1. The smallest width of the microchannel was $100 \mu \mathrm{m}$. Standard photolithography was used for the master mold fabrication and polydimethylsiloxane (PDMS) soft lithography was used for the microfluidic microchannel network fabrication (Fig. 1) ${ }^{21}$ Briefly, a silicon wafer was rinsed with acetone and methanol and baked on a hot plate $\left(150^{\circ} \mathrm{C}\right)$ over 30 minutes for dehydration (Fig. 1(a)). SU-8 photoresist (SU8-2050, Microchem) was spun-coated over the pre-cleaned silicon wafer with a thickness of $100 \mu \mathrm{m}$, and then the wafer was baked on the hot plate at $65^{\circ} \mathrm{C}$ and $95^{\circ} \mathrm{C}$, respectively (Fig. 1 (b)). The designed patterns were transferred from a film mask to the SU-8 thin film after the UV light exposure (OAI model 150, Fig. 1 (c)), post baking, and the development as shown in Fig. 1 (d). After the hard baking at $150^{\circ} \mathrm{C}$, the developed patterns as the master mold were ready for the PDMS soft lithography. The PDMS (Slygard 184, Dow Chemical) was mixed at a weight ratio of $10: 1$, and cast onto the master mold to replicate the microchannel patterns (Fig. 1 e). PDMS was cured and peeled off from the master mold after it was baked in an oven at $60^{\circ} \mathrm{C}$ for 3 hours. The inlet and the outlet, which were used for the cell loading, tubing connections, media and reagent perfusion, and waste collection, were punched with a puncher $(1 \mathrm{~mm}$, Miltex). In a typical confocal microscopy system, the objective lens with high numerical apertures (NA) has a limited working distance in a range of a few hundred microns. ${ }^{22}$ Therefore, to incorporate the microfluidic devices to the confocal system, the number 1 glass coverslip (thickness of 130$160 \mu \mathrm{m}$, Fisher Scientific) spun-coated with a thin layer of PDMS (thickness of $20 \mu \mathrm{m}$ ) was 
used as the substrate for the device bonding (Fig. 1 (f)). A permanent bonding was created to seal the microchannels completely after oxygen plasma treatment (50 W, $100 \mathrm{mtorr}$ ) of PDMS for 30 seconds.

\section{Numerical Simulation of Shear Stress Distribution}

To analyze shear stress distributions inside the microchannel networks, a fluid dynamic analysis model using COMSOL multiphysics software (Version 4.0.0.982, COMSOL Inc.) was built to numerically simulate shear flows. ${ }^{21}$ Incompressible Navier-Stokes equations were chosen as governing equations for the fluids, and no-slip wall boundary condition was set along the internal surface of the microchannels except for the inlet and the outlet.

\section{Cell Culture}

Primary human umbilical vein endothelial cells (HUVECs) were purchased from Lonza. The cells were maintained in MCDB 131 culture medium (Gibco, Life Technologies) supplemented with $10 \%$ fetal bovine serum, $1 \%$ L-glutamine, $0.1 \%$ Gentamicin, $0.05 \%$ bovine brain extract $(9 \mathrm{mg} / \mathrm{mL}), 0.25 \%$ endothelial cell growth supplement $(3 \mathrm{mg} / \mathrm{mL})$, and $0.1 \%$ heparin $(25 \mathrm{mg} / \mathrm{mL})$ in tissue cultured flasks, which were pre-coated with $0.2 \%$ gelatin. The cell culture was performed in a humidified atmosphere of $5 \% \mathrm{CO}_{2}$ at $37^{\circ} \mathrm{C}$, and the cells between passage 2 and passage 5 were used for this study. When the cultured HUVECs reached confluent, the cells were harvested and re-suspended in $8 \%$ Dextran (mol wt 70,000, Sigma) diluted with MCDB 131 culture medium. Dextran was used to increase the medium viscosity for better controlling cell seeding inside the microchannels.

Prior to cell loading, the device was treated with oxygen plasma for 3-5 minutes to reduce the hydrophobicity of inner surfaces of PDMS microchannels. The device was then loaded with deionized water and sterilized under the UV light exposure for 8 hours in a laminar 
biosafety hood. After UV sterilization, the device was rinsed with $1 \times$ phosphate buffered saline (PBS), coated with fibronectin diluted in $1 \times$ PBS $(100 \mu \mathrm{g} / \mathrm{mL}$, Gibco, Life Technologies), and incubated at $4^{\circ} \mathrm{C}$ inside a refrigerator for overnight. After this, the device was rinsed with $1 \times$ PBS again to remove the fibronectin solution completely, and loaded with cell media. Finally, the device was incubated for 15 minutes at $37^{\circ} \mathrm{C}$ and was ready for cell loading.

To load the cells, a droplet $(10 \mu \mathrm{L})$ of HUVECs was placed at the inlet, and a slow flow was created by either tilting the device, or placing a glass pasteur pipette (the inner diameter of the pipets is around $1.5 \mathrm{~mm}, \mathrm{VWR}$ ) at the outlet. Capillary action through the microchannels was gently introduced by the glass pipette and the cells slowly moved along the media into the channels. The key for a successful cell loading was to control the flow velocity very slowly, otherwise, most of the cells cannot attach uniformly inside the microchannels. After 15-20 minutes incubation in the incubator, the attached cells can be visually confirmed under the microscope. An additional loading can be performed if necessary. After a satisfied cell seeding density was reached, the device was gently rinsed with the media to remove the dextran solution. A complete attachment requires five to six hours. Long-term continuous perfusion was set up by a syringe pump system (Harvard Apparatus) with a steady flow rate of $0.35 \mu \mathrm{L} / \mathrm{min}$. The perfusion can last up to two weeks, and can be adjusted to maintain different flow patterns if necessary. ${ }^{4}$

\section{Confocal Fluorescence Imaging of Intracellular Calcium Concentration}

Fluo-4 was selected for confocal $\mathrm{Ca}^{2+}$ imaging. Image acquisition was conducted on a Leica TCS SL confocal microscope with a Leica $\times 25$ objective (NA: 0.95). An argon laser $(488 \mathrm{~nm})$ at $50 \%$ power was used for excitation, and the emission band was $510-530 \mathrm{~nm}$. 
To minimize photobleaching, fluo-4 images were collected using a $512 \times 512$ scan format at a z-step of $2 \mu \mathrm{m}$. Stacks of images were collected from the same group of HUVECs with 20 seconds intervals. Each network device was first loaded with fluo-4 AM (5 $\mu \mathrm{M})$ for 40 minutes followed by albumin-Ringer perfusion to rinse the lumen fluo-4 AM before control images were collected. Quantitative analysis of endothelial $\left[\mathrm{Ca}^{2+}\right]_{i}$ at the individual endothelial cell level was conducted using manually selected ROls along the microchannels. Each ROI covered the area of one individual endothelial cell, as indicated by the fluorescence outline. The changes in endothelial $\left[\mathrm{Ca}^{2+}\right]$ at the cellular levels were quantified by calculating the mean $\mathrm{FI}$ of each stack of ROls after the subtraction of the background auto fluorescence. The percent change in $\mathrm{FI}$ was expressed as $\mathrm{Fl} / \mathrm{Fl}_{0}{ }^{*} 100$, where $\mathrm{Fl}_{0}$ was the initial baseline $\mathrm{FI}$ of fluo-4. Details have been described previously. ${ }^{25}$

\section{Confocal Fluorescence Imaging of Nitric Oxide Production}

Endothelial NO levels were investigated at the cellular levels in the microvessel network using DAF-2 DA, a membrane-permeable fluorescent indicator for NO, and fluorescence imaging. Experiments were performed on a Nikon Diaphod 300 microscope equipped with a 12-bit digital CCD camera (ORCA; Hamamatsu) and a computer controlled shutter (Lambda 10-2; Sutter Instrument; Novato, CA). A 75-W xenon lamp was used as the light source. The excitation wavelength for DAF-2 was selected by an interference filter (480/40 $\mathrm{nm})$, and emission was separated by a dichroic mirror $(505 \mathrm{~nm})$ and a band-pass barrier (535/50 nM). All the images were acquired and analysed using Metafluor software (Universal Imaging).

Each network device was first perfused with albumin-Ringer solution containing DAF-2 DA $(5 \mu \mathrm{M})$ for $35 \sim 40$ minutes before collecting DAF-2 images. DAF-2 DA was present in 
the perfusate throughout the experimental duration. All images were collected from a group of HUVECs located in the same focal plane using a Nikon Fluor lens (x20, NA: 0.75). Data analysis was conducted at the individual endothelial cell level using manually selected regions of interests (ROIs). Each $\mathrm{ROI}$ covered the area of one individual cell as indicated by the fluorescence outline. The PDMS auto fluorescence was subtracted from all of the measured fluorescence intensities (Fls). The basal NO production rate was calculated from the slope of the mean $\mathrm{Fl}$ increase during albumin-Ringer perfusion after DAF-2 loading was reached the steady state. The changes in FlDAF upon ATP stimulation were expressed as the net changes in $\mathrm{FI}(\Delta \mathrm{FI})$. $\mathrm{FI}$ was expressed in arbitrary units $(\mathrm{AU})$ measured with identical instrumental settings. The rate of Fl DAF change was derived by first differential conversion of cumulative FlDAF over time. Details have been described previously. ${ }^{23,} 24$

\section{Immunofluorescent Staining}

HUVECs were fixed in $2 \%$ paraformaldehyde solution (Electron Microscopy Science) for 30 minutes by perfusing the fixing solution into the network. The cells were blocked with $1 \mathrm{mg} / \mathrm{mL}$ bovine serum albumin (BSA, Sigma) in PBS solution for 30 minutes followed by permeabilization with $0.1 \%$ Triton X-100 (Sigma) for 5 minutes. The primary antibody (VEcadherin) was perfused at $4{ }^{\circ} \mathrm{C}$ for overnight. Then, the second antibody (Alexa488, Invitrogen) was perfused for 1 hour at the room temperature. Finally, the cell nuclei were stained with DRAQ 5 (Biostatus). The F-actin staining followed the similar fixing, blocking, and permeabilizing procedures, and then stained with phalloidin-Alexa 633 (Sigma) for 10 minutes, followed by the DRAQ5 nuclei staining. Fluorescent images were obtained using an inverted optical microscope (Nikon Ti-E inverted live-cell imaging microscope) and a confocal laser-scanning microscope (Leica TCS SL), respectively. 


\section{Cell Morphology Analysis in Response to Shear Stress}

To study the actin cytoskeleton and HUVECs morphology changes under shear stresses, different scenarios were performed to vary the culture and shear flow conditions. Detailed experimental conditions are listed in Table 1. Briefly, after initial seeding three different flow conditions were set for the same patterned networks in different devices as shown in Table 1: Low shear culture, low shear test (LSC-LST); Low shear culture (till the ECs reached confluence), high shear test (LSC-HST); and high shear culture, high shear test (HSC-HST). The transition from low shear stress to high shear stress was gradually applied by programming a step function (10 steps of increase in 18 hours) using the syringe pump (Harvard Apparatus).

Quantitative analysis was performed to examine HUVEC morphology changes (i.e. aspect ratio and cell surface area) in responses to different levels of shear stresses. The aspect ratio was defined as the length of the primary axis $\left(\mathrm{d}_{1}\right.$, along the flow direction) divided by the length of the secondary axis ( $d_{2}$, perpendicular to the flow direction). A cell was considered as an ellipse shape and the corresponding cell surface area was calculated as $\frac{\pi d_{1} d_{2}}{4}$.

\section{Data analysis and statistics}

For statistical analysis, data was presented as the mean \pm standard error (SE) and each individual experiment was performed at least three times $(n \geq 3)$. Statistical significance of detected difference between groups was tested using the single-factor ANOVA. 


\section{RESULTS}

\section{Characterization of the Endothelial Adherens Junctions in Microvessels Developed in Microchannel Network}

With the initial cell loading concentrations of $2 \sim 4 \times 10^{6} \mathrm{cell} / \mathrm{sL}$, confluent monolayers developed within 3-4 days under a constant flow of culture media. Fig. 2 shows the confocal images of endothelial cell F-actin and cell nuclei staining at different regions of the network in the same device, which illustrates that HUVECs successfully cultured the entire inner surface of the network to confluence. We also examined the junctional formation between ECs as an indication of endothelial barrier function. VE-cadherin, an important protein for the maintenance and control of the junctions between endothelial cells, was immunestained in the entire microchannel. Fig. 3 shows the confocal images of VE-cadherin and nuclei staining at different regions of the microchannel. The confocal images illustrate that VE-cadherin was well developed throughout the entire network (Fig. 3 b-d), demonstrating a continuous distribution between ECs with less lattice-like structure that often appeared in statically cultured endothelial monolayers. ${ }^{26}$ The VE-cadherin distribution in the microchannels is closer to that demonstrated in intact microvessels, ${ }^{12}$ suggesting that the continuous flow condition during cell growth provide a better environment for appropriate EC spreading, viability, proliferation, and formation of junctions.

\section{Endothelial Cell Responses to Shear Stress}

To quantify the shear stress, which the endothelium lining the microchannels experienced under our experimental conditions, we conducted a numerical simulation. The calculated wall shear stresses were 1.0 dyne $/ \mathrm{cm}^{2}$ under the flow rate $0.35 \mu \mathrm{L} / \mathrm{min}$, and 10 
dyne/cm2 under the flow rate $4.05 \mu \mathrm{L} /$ min within the selected regions in the devices as shown in Fig. 4a-b.

Flow related shear stress has been shown to induce changes in cell shape and cytoskeletal structure of vascular ECs in vivo and in vitro. ${ }^{27,28}$ In this study, we evaluated the cytoskeletal rearrangement of F-actin fibers and cell shape changes in response to three patterns of flow related shear stress within the microchannel networks: continuous low shear without a change (LSC-LST); low shear culture with high shear exposure (LSCHST); and continuous high shear exposure (HSC-HST). The flow rate and correlated shear stress under each condition are listed in Table 1. Under the LSC-LST conditions, about $70 \%$ of the cells show cobblestone pattern with dominated peripheral F-actin, and only $30 \%$ of the HUVECs showed elongated cell shape with increased central stress fibers aligned along the flow direction. Under LSC-HST and HSC-HST conditions, about $50 \%$ of the cells were elongated with distinct stress fibers along the flow direction. In each scenario, the cell shape aspect ratio in non-aligned cells was about $1.5 \pm 0.03$ and the aligned cells was 3.97 $\pm 0.19,3.93 \pm 0.22$ and $3.95 \pm 0.19$ in LSC-LST, LSC-HST, and HSC-HST, respectively. Fig. 4c-g shows representative images from each group and the quantifications of their changes in morphology, including aspect ratio and cell surface areas, in those aligned and non-aligned cells, respectively. The aligned cells in high shear stress test groups (LSCHST and HSC-HST) demonstrated significantly reduced surface areas $\left(1855 \mu \mathrm{m}^{2}\right.$ and 1234 $\mu \mathrm{m}^{2}$ ) compared to those cells under low shear stress conditions (LSC-LST, $2202 \mu \mathrm{m}^{2}$, Fig. $4)$. 


\section{Measurements of Endothelial Calcium Concentration $\left(\left[\mathrm{Ca}^{2+}\right]_{i}\right)$ in response to ATP}

Increases in endothelial $\left[\mathrm{Ca}^{2+}\right]_{i}$ have been demonstrated to play important roles in the regulation of a variety of microvessel functions including endothelial barrier function, i.e. microvessel permeability. In individually perfused microvessels, inflammatory mediator commonly induces transient increases in endothelial $\left[\mathrm{Ca}^{2+}\right]_{i}$ followed by transient increases in microvessel permeability. To validate the biological functions of the microvessels developed under flow in our model, we applied the method developed in individually perfused intact microvessels ${ }^{25,29}$ to the microvessels developed in the microchannel network and quantitatively measured the changes in endothelial $\left[\mathrm{Ca}^{2+}\right]_{i}$ when the microvessel was exposed to ATP. Experiments were conducted in four devices with fluo-4 loaded endothelial cells. When ATP $(10 \mu \mathrm{M})$ was perfused to each device, $\left[\mathrm{Ca}^{2+}\right]_{i}$ in all endothelial cells under the view reached a peak at $35 \pm 10$ seconds with the mean peak $\left[\mathrm{Ca}^{2+}\right]$ at $187 \pm 22 \%$ of the control. Representative fluo- 4 images and the fluorescence quantification are shown in Fig. 5.

\section{Measurement of ATP-induced Nitric Oxide Production}

In intact venules, it has been demonstrated that inflammatory mediator-induced increases in endothelial $\left[\mathrm{Ca}^{2+}\right]$ i was associated with increased NO production, and both increased endothelial $\left[\mathrm{Ca}^{2+}\right]_{\mathrm{i}}$ and NO production contribute to increases in microvessel permeability. ${ }^{24,} 25$ With demonstrated ATP-induced increases in endothelial $\left[\mathrm{Ca}^{2+}\right]$, we further measured NO production in response to ATP in DAF-2 loaded endothelial cells lining the microchannels.

When ATP $(10 \mu \mathrm{M})$ was added, the rate change in ATP-induced NO production was measured with continuous DAF-2 DA perfusion. ${ }^{23}$ Each device was first perfused with 
albumin-Ringer solution containing DAF-2 DA $(5 \mu \mathrm{M})$ for 35 40 minutes before collecting images. After the loading phase, the rate change of FlDAF under control conditions was 0.15 $\pm 0.05 \mathrm{AU}$ per min, which was close to the basal NO production rate measured in intact rat mesenteric venules. ${ }^{10,23,30}$ When ATP $(10 \mu \mathrm{M})$ was added to the perfusate, the rate change in FlDAF was significantly increased. The peak rate of Fl DAF increase was $1.18 \pm 0.37 \mathrm{AU}$ per min. The increased FlDAF returned to the control level after 10 15 minutes of ATP exposure. Data were derived from 3 devices with a total of 35 ROls and 11 to 12 ROls per vessel. NO donor, sodium nitroprusside (SNP), was added at the end of each experiment to verify the sufficiency of DAF-2 in the cells and the responsiveness of DAF-2 to NO. Fig. 6 shows the quantification of time-dependent changes in FlDAF and correlated images from an individual experiment. The changes in slopes of the Fl DAF indicate the changes in NO production rates before and after ATP stimulation. Images in Fig. 6 (b) illustrate the ATPinduced cumulated increases in FlDAF at 10 minutes of ATP exposure. 


\section{DISCUSSION}

Our study presented the in vitro formation of a microvessel network using a microfluidic device and characterized endothelial junctions, cellular responses to shear stresses, and quantitatively measured the changes in endothelial $\left[\mathrm{Ca}^{2+}\right]_{i}$ and NO production before and after exposure to ATP. The results demonstrated that this engineered microvessel network recapitulates the key aspects and fundamental functions of in vivo venules, and reveals its promising utility for biological applications including studies with human samples.

With advanced microfabrication techniques, the flexibility to create patterns and scale sizes provides users with arbitrary design options to mimic the geometries of in vivo microvasculature, which allows the functional studies involved in complex flow patterns found at vessel bifurcations and in regions of high curvature that occur in vivo. This microfluidic microvessel network with thin PDMS film layer bonded on a thin glass substrate (average total thickness is $170 \mu \mathrm{m}$ ) allows excellent light transmission and easy adaptation to both bright field and fluorescence microscopy when using short working distance and large numerical aperture objectives. Our study demonstrated that this device is capable for real-time and high-resolution imaging to detect changes in intracellular molecules and cell morphology using fluorescent markers, which is a necessary feature for biological studies. This modification is a further progress of applying our previous studies using endothelialized microvessel network. ${ }^{4,21}$

The main difference of using this microchannel network compared with traditional cell culture is that the cells, instead of growing under static conditions at 2-dimensional flat surface, were growing in a three dimensional channel with continuous flow, which made the cell culture environment closer to the in vivo situation. The numerical simulation 
provides an accurate estimation of the wall shear stress distribution. Based on the channel dimensions and simulation, the applied shear stress was easily controlled within the range of venous system ( 1 to $\left.10 \mathrm{dyne} / \mathrm{cm}^{2}\right) .31,32$ Our study characterized the biological functions of these cultured microvessels under basal and stimulated conditions, and validated its utility for biological applications. Our results indicated that the endothelial cells grown under flow conditions form better monolayers with well-developed junctions between endothelial cells as that demonstrated by VE-cadherin staining. As shown in Fig. 2, the HUVECs completely covered the entire inner surfaces of the channels including the corners and formed a completely enclosed network. Most importantly, the VE-cadherin staining showed a uniformly distributed band between endothelial cells within the network, which is similar to that observed in intact venules, ${ }^{12,33}$ and distinct from the intricate lattice-like structure commonly found in statically cultured endothelial monolayers. ${ }^{34}$

The major technical improvement for biological and physiological application reported in this study is the quantitative measurements of agonist-induced changes in endothelial $\left[\mathrm{Ca}^{2+}\right]_{i}$ and NO production at individual cell levels in a well-developed microvessel network. Studies in individually perfused intact microvessels indicated that inflammatory mediatorinduced increases in endothelial $\left[\mathrm{Ca}^{2+}\right]_{i}$ is essential for increases in microvessel permeability and that the magnitude of the $\left[\mathrm{Ca}^{2+}\right]$ determines the degree of permeability increases. ${ }^{29,45-48}$ In addition, agonist-induced $\mathrm{Ca}^{2+} /$ calmodulin-dependent endothelial nitric oxide synthase(eNOS) activation and NO production have been shown to play important roles in the regulation of microvessel permeability. ${ }^{24,25,49}$ In this study, we directly applied the methods developed in individually perfused intact microvessels ${ }^{23,29}$ to this cultured microvessel network and conducted parallel studies to those performed in the intact 
microvessels in vivo. Currently the uses of microfluidics to study intracellular calcium ${ }^{50,51}$ and NO responses ${ }^{52}$ have been reported by a few investigators. However, the calcium studies were either from suspended leukemic cells ${ }^{50}$ or from non-confluent osteoblasts. ${ }^{51}$ Though ATP-induced NO has been reported in BPAEC cultured microfluidic device, there was no characterization of the junctions of endothelial cells in which NO was measured and no temporal resolution of NO production was analysed in that study. ${ }^{52}$ In our study, we choose ATP as the representative agonist to study receptor-mediated changes in endothelial $\left[\mathrm{Ca}^{2+}\right]$ and NO production. ATP can be released by red blood cells, aggregated platelets, and injured tissue or under inflammatory conditions. The increased levels of ATP cause the release of endothelial-derived relaxing factor and trigger the synthesis of prostacyclin, and increase in microvessel permeability by increasing endothelial $\left[\mathrm{Ca}^{2+}\right]_{1 .}{ }^{48}$, 53, 54 The action of ATP is primarily via the purinergic $\mathrm{P}_{2 y}$ receptor expressed on most types of endothelial cells. ${ }^{55,56}$ The calcium measurements using fluo-4 as indicator in the microvessel network showed similar responses to what we found in intact microvessels. ${ }^{25}$, ${ }^{48}$ As for NO measurements using DAF-2 DA, we need to recognize the specific manner of DAF-2 chemical conversion in the presence of $\mathrm{NO}$ and conduct data analysis accordingly. ${ }^{23,57}$ DAF-2 DA is membrane permeable and diffuses freely into the cells driven by concentration gradient and is then hydrolyzed by cytosolic esterase to form DAF-2. Intracellular DAF-2 is less membrane permeable due to its polarity, and is designed to be trapped inside the cells. Because the fluorescence chemical transformation of DAF-2 by $\mathrm{NO}$ is irreversible, ${ }^{57}$ the detected NO-sensitive fluorescence with DAF-2 represents a cumulative production of NO. The slope of the Fl DAF curve represents the NO production rate and the plateau indicates the termination of NO production instead of the actual NO 
concentration in the cells. To present the real-time course of NO production, $\mathrm{df} / \mathrm{d} t$, the NO production rate, was calculated based on the differential conversion of that regression equation (shown in Fig. 5a). Using this method, our study presented the basal NO production rate and the changes in NO production after exposure to ATP in HUVECsdeveloped microvessels in the microfluidic device with temporal and spatial resolution for the first time. The results are comparable to those derived from individually perfused intact venules. These studies provide an in vivo validation of the functional aspect of engineered microvessel network, which is instrumental for the utility of this device to the development of biological applications. 


\section{CONCLUSIONS}

These results demonstrated in vitro formation of a microvessel network that recapitulates key features of microvessels in vivo and validate its utility for biological applications. The fabrication process of the microchannels was simple and straightforward to lower the barrier for biologists. The cell seeding and culture with constant perfusion method was user friendly to most researchers and easy to replicate. Cells in our model keep their phenotype, viability, proliferation with proper barrier functions, and respond to flow shear force and inflammatory stimuli. Comparing to other conventional in vitro models, our model is more physiological realistic. In particular, HUVECs were successfully grown to confluence throughout entire microchannels under well-controlled conditions. The model, including in vitro growth endothelialized microchannels and the flow control, mimics microscaled dimensions of in vivo microvessels and continuous flow patterns. Immunofluorescent staining of VE-cadherin demonstrated the formation of vascular endothelial junctions, an indication of well-maintained endothelial restrictive barrier functions. HUVECs responded to different physiologically relevant flow shear stresses and inflammatory stimuli. The endothelial $\left[\mathrm{Ca}^{2+}\right] \mathrm{i}$, basal $\mathrm{NO}$, and the changes in NO production rate in response to ATP were real-time measured and quantified at individual cellular level in Fluo 4 and DAF-2 loaded microvessels, respectively. The transient $\left[\mathrm{Ca}^{2+}\right]_{\mathrm{i}}$ responses and increased NO production in response to agonist application are similar to those observed in individually perfused intact microvessels. The developed microvessel model has the potential to bridge the gap between over-simplified in vitro tests and more expensive and labour-intensive in vivo animal models for the microvascular signalling and functional studies. It offers valuable quantitative insights into how biophysical and biochemical 
properties influence vascular biology and pathophysiology, serves as the complements for in vivo animal studies. Because the cultured endothelial cells can be from human tissues, a well characterized device will be suitable for studies of interactions between human blood components with microvessels formed by human endothelial cells under physiological and pathological conditions.

\section{Acknowledgements}

This work was supported by the National Science Foundation (NSF-1227359) and by WV EPSCoR program (EPS-1003907) funded by the National Science Foundation (Liu, Y.); and by National Heart, Lung, and Blood Institute grants HL56237 and HL084338 (He, P.) and American Heart Association Great Rivers Affiliate 12PRE11470010 pre-doctoral fellowship (Xu, S.). The microfabrication work was performed in Shared Research Facilities (Cleanroom facilities) and Microfluidic Integrative Cellular Research on Chip Laboratory (MICRoChip Lab) at West Virginia University. 


\section{REFERENCE}

1. E. K. Sackmann, A. L. Fulton and D. J. Beebe, Nature, 2014, 507, 181-189.

2. E. Berthier, E. W. Young and D. Beebe, Lab on a Chip, 2012, 12, 1224-1237.

3. J.-J. Chiu and S. Chien, Physiological reviews, 2011, 91, 327-387.

4. X. Li, S. Mearns, M. Martins-Green and Y. Liu, Journal of visualized experiments: JoVE, 2012, e50771-e50771.

5. J. El-Ali, P. K. Sorger and K. F. Jensen, Nature, 2006, 442, 403-411.

6. G. M. Price, K. H. Wong, J. G. Truslow, A. D. Leung, C. Acharya and J. Tien, Biomaterials, 2010, 31, 6182-6189.

7. Y. Zheng, J. Chen, M. Craven, N. W. Choi, S. Totorica, A. Diaz-Santana, P. Kermani, B. Hempstead, C. Fischbach-Teschl and J. A. López, Proceedings of the National Academy of Sciences, 2012, 109, 9342-9347.

8. M. Tsai, A. Kita, J. Leach, R. Rounsevell, J. N. Huang, J. Moake, R. E. Ware, D. A. Fletcher and W. A. Lam, The Journal of clinical investigation, 2012, 122, 408-418.

9. L. L. Bischel, E. W. Young, B. R. Mader and D. J. Beebe, Biomaterials, 2013, 34, 1471 1477.

10. S. Xu, X. Zhou, D. Yuan, Y. Xu and P. He, American Journal of Physiology-Heart and Circulatory Physiology, 2013, 305, H1484-H1493.

11. M. H. Zadeh, C. A. Glass, A. Magnussen, J. C. Hancox and D. O. Bates, Microcirculation, 2008, 15, 605-614.

12. D. Yuan and P. He, Journal of Applied Physiology, 2012, 113, 1110-1120.

13. L. Munaron, Recent patents on anti-cancer drug discovery, 2006, 1, 105-119.

14. M.-J. Lee, S. Thangada, K. P. Claffey, N. Ancellin, C. H. Liu, M. Kluk, M. Volpi, R. I. Sha'afi and T. Hla, Cell, 1999, 99, 301-312.

15. T. D. Oblak, P. Root and D. M. Spence, Analytical Chemistry, 2006, 78, 3193-3197.

16. S. Letourneau, L. Hernandez, A. N. Faris and D. M. Spence, Anal Bioanal Chem, 2010, 397, 3369-3375.

17. M. Tsai, A. Kita, J. Leach, R. Rounsevell, J. N. Huang, J. Moake, R. E. Ware, D. A. Fletcher and W. A. Lam, J Clin Invest, 2012, 122, 408-418.

18. S. Kim, H. Lee, M. Chung and N. L. Jeon, Lab Chip, 2013, 13, 1489-1500.

19. B. Melchior and J. A. Frangos, Am J Physiol Cell Physiol, 2012, 303, C467-473.

20. M. A. Corson, N. L. James, S. E. Latta, R. M. Nerem, B. C. Berk and D. G. Harrison, Circ Res, 1996, 79, 984-991.

21. Z. Huang, X. Li, M. Martins-Green and Y. Liu, Biomedical microdevices, 2012, 14, 873-883.

22. H. E. Keller, in Handbook Of Biological Confocal Microscopy, ed. J. B. Pawley, Springer US, 2006, pp. 145-161.

23. X. Zhou and P. He, American Journal of Physiology-Heart and Circulatory Physiology, 2011, 301, H108-H114. 
24. L. Zhu and P. He, American Journal of Physiology-Heart and Circulatory Physiology, 2005, 288, H2869-H2877.

25. X. Zhou and P. He, American Journal of Physiology-Heart and Circulatory Physiology, 2011, 301, H1788-H1797.

26. F. Orsenigo, C. Giampietro, A. Ferrari, M. Corada, A. Galaup, S. Sigismund, G. Ristagno, L. Maddaluno, G. Y. Koh, D. Franco, V. Kurtcuoglu, D. Poulikakos, P. Baluk, D. McDonald, M. Grazia Lampugnani and E. Dejana, Nat Commun, 2012, 3, 1208.

27. B. L. Langille, J. J. Graham, D. Kim and A. I. Gotlieb, Arterioscler Thromb, 1991, 11, 1814-1820.

28. M. Sato and T. Ohashi, Biorheology, 2005, 42, 421-441.

29. P. He, S. N. Pagakis and F. E. Curry, Am J Physiol, 1990, 258, H1366-1374.

30. X. Zhou, D. Yuan, M. Wang and P. He, American Journal of Physiology-Heart and Circulatory Physiology, 2013, 304, H82-H93.

31. H. H. Lipowsky, in Flow-Dependent Regulation of Vascular Function, Springer, 1995, pp. 28-45.

32. A. M. Malek, S. L. Alper and S. Izumo, Jama, 1999, 282, 2035-2042.

33. D. Yuan, S. Xu and P. He, Am J Physiol Heart Circ Physiol, 2014, 307, H44-53.

34. J. A. Ukropec, M. K. Hollinger and M. J. Woolkalis, Experimental cell research, 2002, 273, 240-247.

35. E. Tzima, M. Irani-Tehrani, W. B. Kiosses, E. Dejana, D. A. Schultz, B. Engelhardt, G. Cao, H. DeLisser and M. A. Schwartz, Nature, 2005, 437, 426-431.

36. T. Nagel, N. Resnick, W. J. Atkinson, C. F. Dewey Jr and M. A. Gimbrone Jr, Journal of Clinical Investigation, 1994, 94, 885.

37. H. Morawietz, R. Talanow, M. Szibor, U. Rueckschloss, A. Schubert, B. Bartling, D. Darmer and J. Holtz, The Journal of physiology, 2000, 525, 761-770.

38. P. Feugier, R. Black, J. Hunt and T. How, Biomaterials, 2005, 26, 1457-1466.

39. L. Chau, M. Doran and J. Cooper-White, Lab Chip, 2009, 9, 1897-1902.

40. B. R. Blackman, G. Garcia-Cardena and M. A. Gimbrone, Jr., J Biomech Eng, 2002, 124, 397-407.

41. D. E. Berardi and J. M. Tarbell, Cell Mol Bioeng, 2009, 2, 320-331.

42. Y. Xia, M. Prawirasatya, B. C. Heng, F. Boey and S. S. Venkatraman, Journal of materials science: Materials in medicine, 2011, 22, 389-396.

43. D. M. McDonald, American Journal of Physiology-Lung Cellular and Molecular Physiology, 1994, 10, L61.

44. F. Orsenigo, C. Giampietro, A. Ferrari, M. Corada, A. Galaup, S. Sigismund, G. Ristagno, L. Maddaluno, G. Y. Koh and D. Franco, Nature communications, 2012, 3, 1208.

45. F. E. Curry, FASEB J, 1992, 6, 2456-2466.

46. P. He and F. E. Curry, Am J Physiol, 1991, 261, H1246-1254.

47. P. He and F. E. Curry, J Appl Physiol (1985), 1994, 76, 2288-2297. 
48. P. He, X. Zhang and F. E. Curry, Am J Physiol, 1996, 271, H2377-2387.

49. X. Zhou and P. He, Cardiovasc Res, 2010, 87, 340-347.

50. T. Xu, W. Yue, C. W. Li, X. Yao and M. Yang, Lab Chip, 2013, 13, 1060-1069.

51. S. Kou, L. Pan, D. van Noort, G. Meng, X. Wu, H. Sun, J. Xu and I. Lee, Biochem Biophys Res Commun, 2011, 408, 350-355.

52. T. D'Amico Oblak, P. Root and D. M. Spence, Anal Chem, 2006, 78, 3193-3197.

53. R. S. Sprague, J. J. Olearczyk, D. M. Spence, A. H. Stephenson, R. W. Sprung and A. J. Lonigro, Am J Physiol Heart Circ Physiol, 2003, 285, H693-700.

54. R. J. Mannix, T. Moatter, K. A. Kelley and M. E. Gerritsen, Am J Physiol, 1993, 265, H675-680.

55. G. R. Dubyak and C. el-Moatassim, Am J Physiol, 1993, 265, C577-606.

56. T. D. Carter, T. J. Hallam, N. J. Cusack and J. D. Pearson, Br J Pharmacol, 1988, 95, 1181-1190.

57. H. Kojima, N. Nakatsubo, K. Kikuchi, S. Kawahara, Y. Kirino, H. Nagoshi, Y. Hirata and T. Nagano, Anal Chem, 1998, 70, 2446-2453. 
(a)

(c)
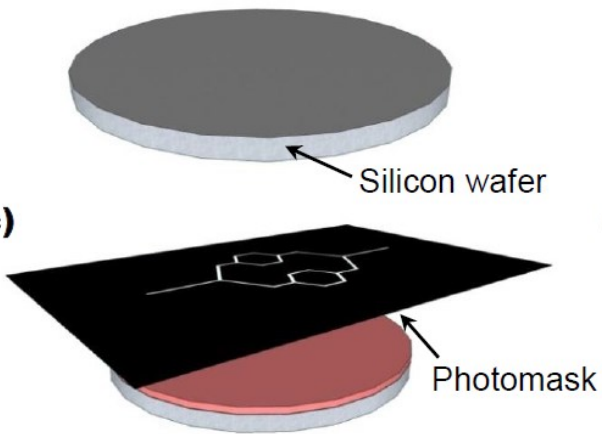

(e)

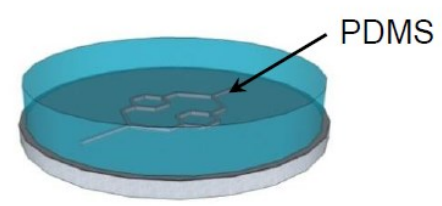

(b)

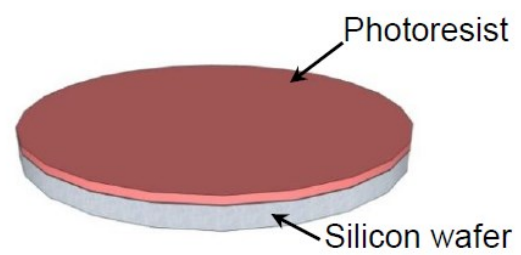

(d)

Developed photoresist

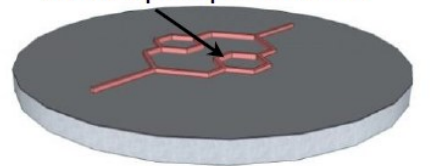

(f)

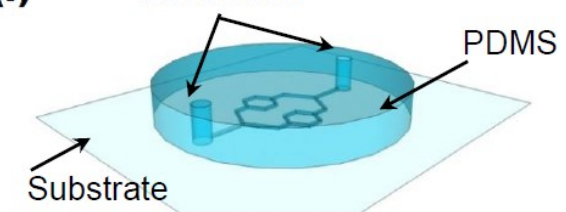

Figure. 1 The schematic fabrication procedures for the microfluidic microchannel network. (a) A pre-cleaned silicon substrate; (b) SU-8 photoresist was spun-coated onto the silicon wafer; (c) The photoresist was exposed to UV light through the photomask; (d) The developed microchannel network pattern was used as the master mold; (e) The PDMS mixing solution was cast onto the master mold and cured; (f) The inlet and the outlet were punched and the microchannel device was bonded onto the substrate. 
Fig.2
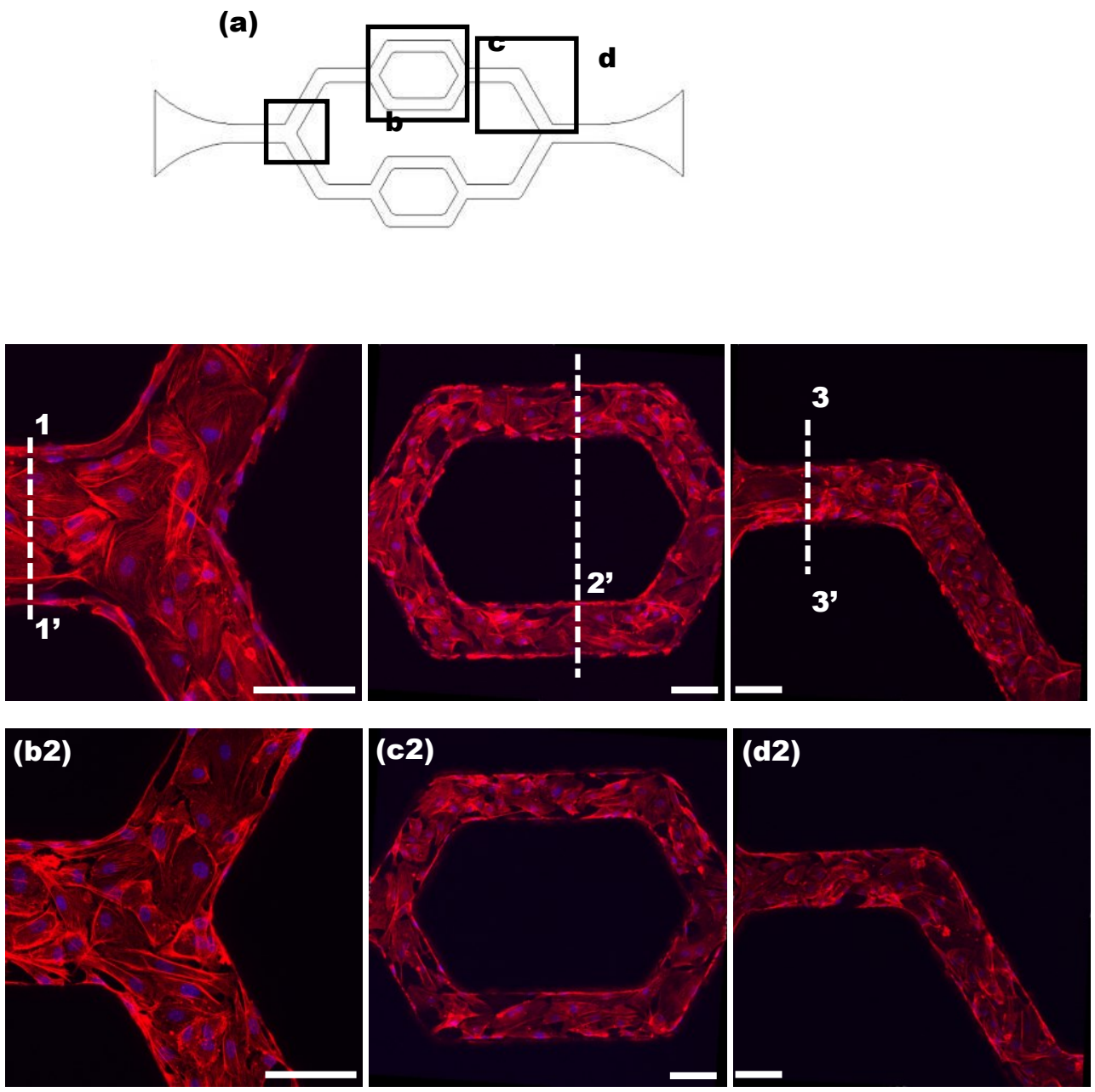

(e)

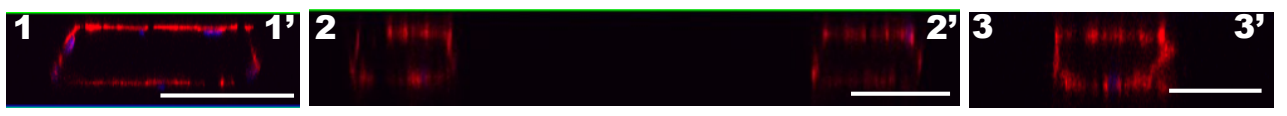


Figure. 2 The representative confocal images show the HUVECs successfully cultured throughout the inner surfaces of the entire microchannel network. (a) The schematic image of the network with selected regions as shown in b-d; (b-d) HUVECs were stained with F-actin and cell nuclei in each region, where b1, c1 and d1 show the top surfaces of the channel, respectively. b2, $c 2$ and d2 show the bottom surfaces of the channel, respectively; (e) The cross-sectional images at each region. The locations of the cross-sections were indicated as 1-1', 2-2', and 3-3' in b1-d1, respectively. Scale bars: $100 \mu \mathrm{m}$. 
Fig.3

(a)
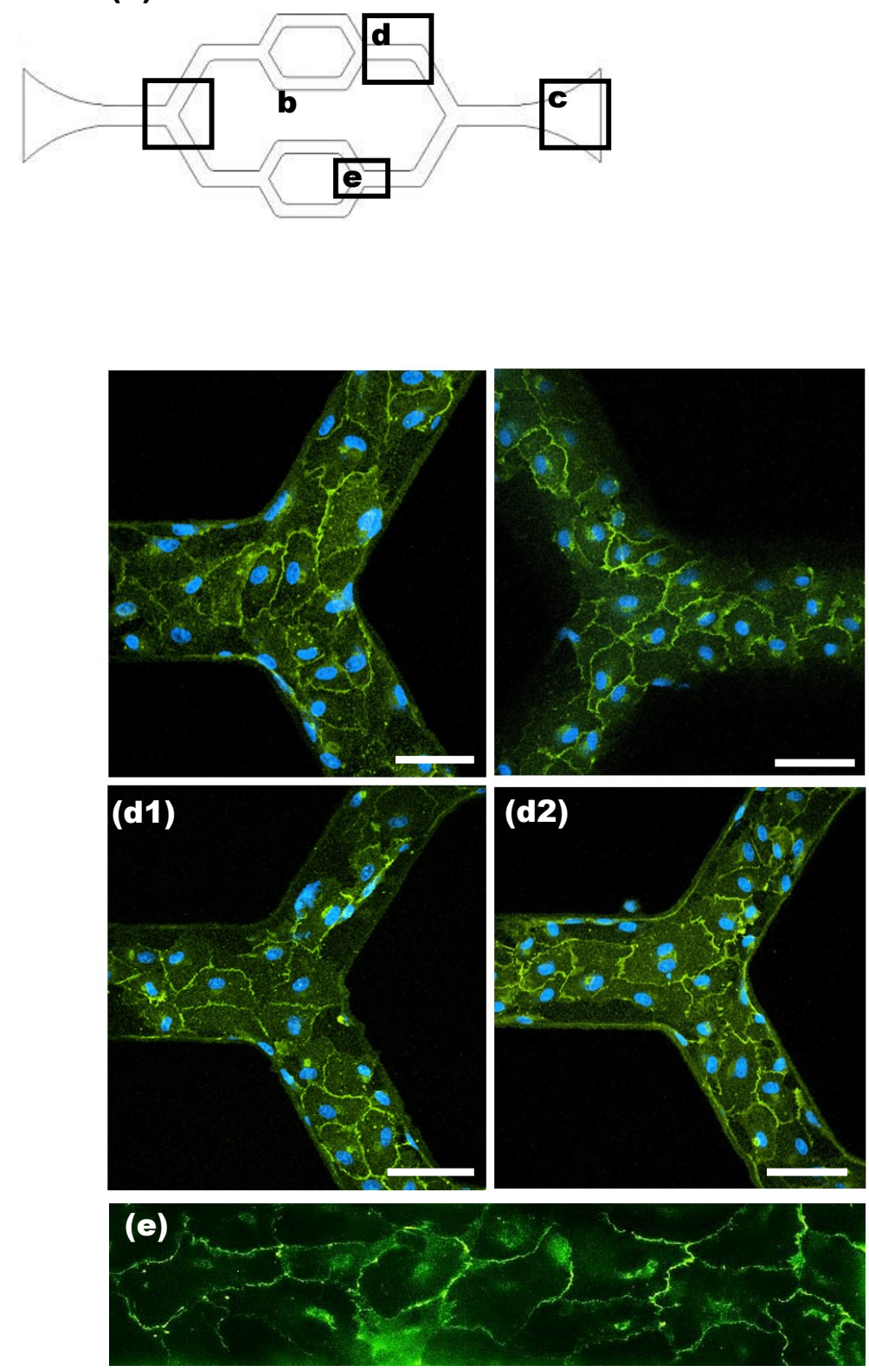
Figure. 3 The representative immunofluorescent confocal images demonstrated the VE-Cadherin junctions formation throughout the entire network. (a) The schematic image of the network with selected regions as shown in b-d; (b-c) VE-Cadherin and cell nuclei staining were shown at the first and third branching regions; (d1-d2) VECadherin and cell nuclei staining were shown at the top and bottom surfaces of the second branching regions, respectively; (e) Enlarged region indicated in (a). Scale bars: $100 \mu \mathrm{m}$ 
Fig.4

(a)

(b)
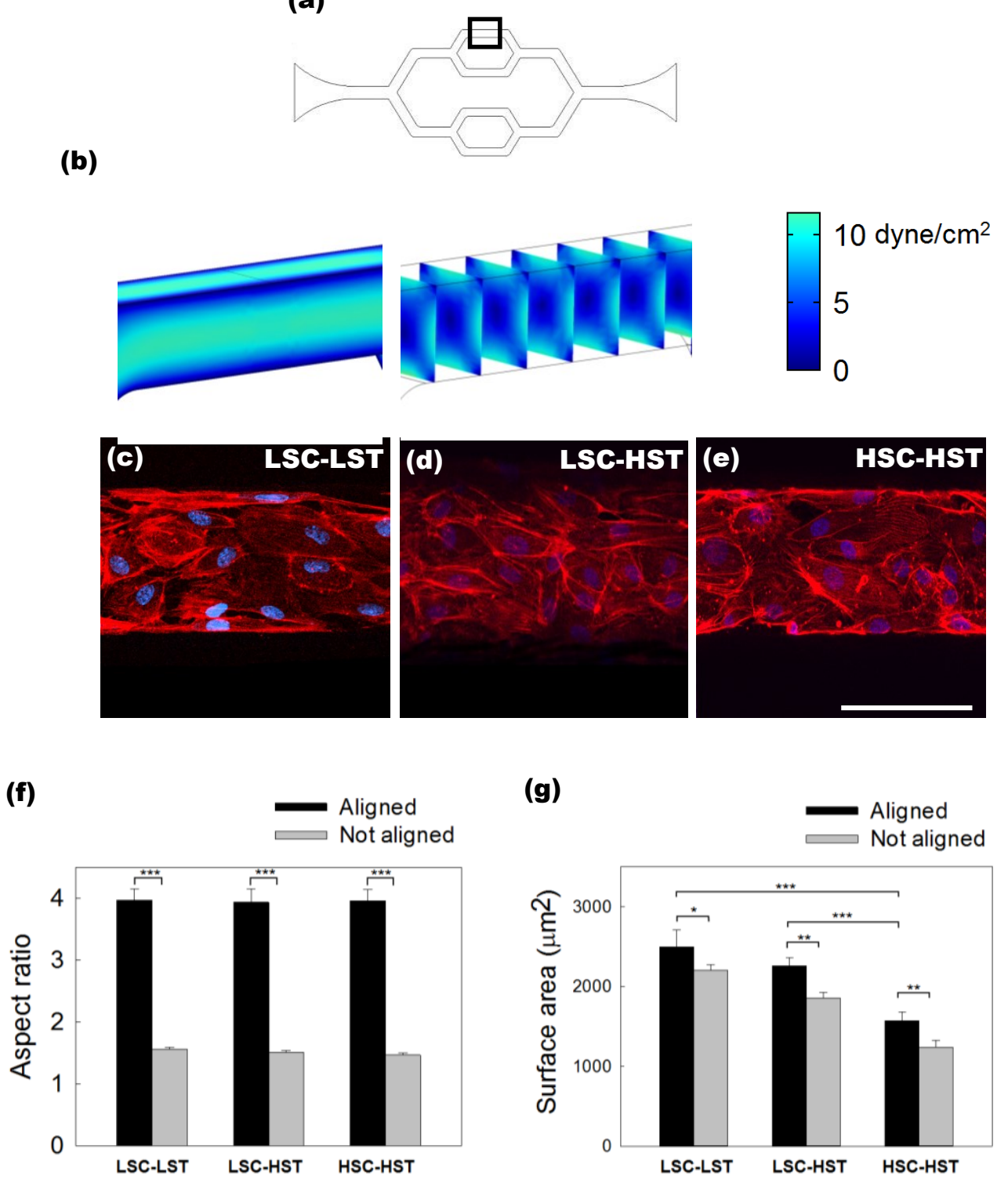
Figure. 4 HUVECs response to the different levels of shear stresses: The morphology change was studied with F-actin staining. (a) The schematic image of the network with the indicated selected region for shear stress test; (b) COMSOL simulation shows the wall shear stress distribution in the selected region along the network. (c-e) Confocal image showed the F-actin stress fibers under the LSC-LST, LSCHST, and HSC-HST respectively. (f) Aspect ratio comparing between the three testing groups; (g) Comparing of the cell surface areas between the testing groups; All data are reported as the means \pm SE of independent experiment. Scale bars: $100 \mu \mathrm{m}$. *: $P<0.05$; ${ }^{* *}: \mathrm{P}<0.01 ;{ }^{* * *}: \mathrm{P}<0.001$ 
Fig.5

(a)

(b)
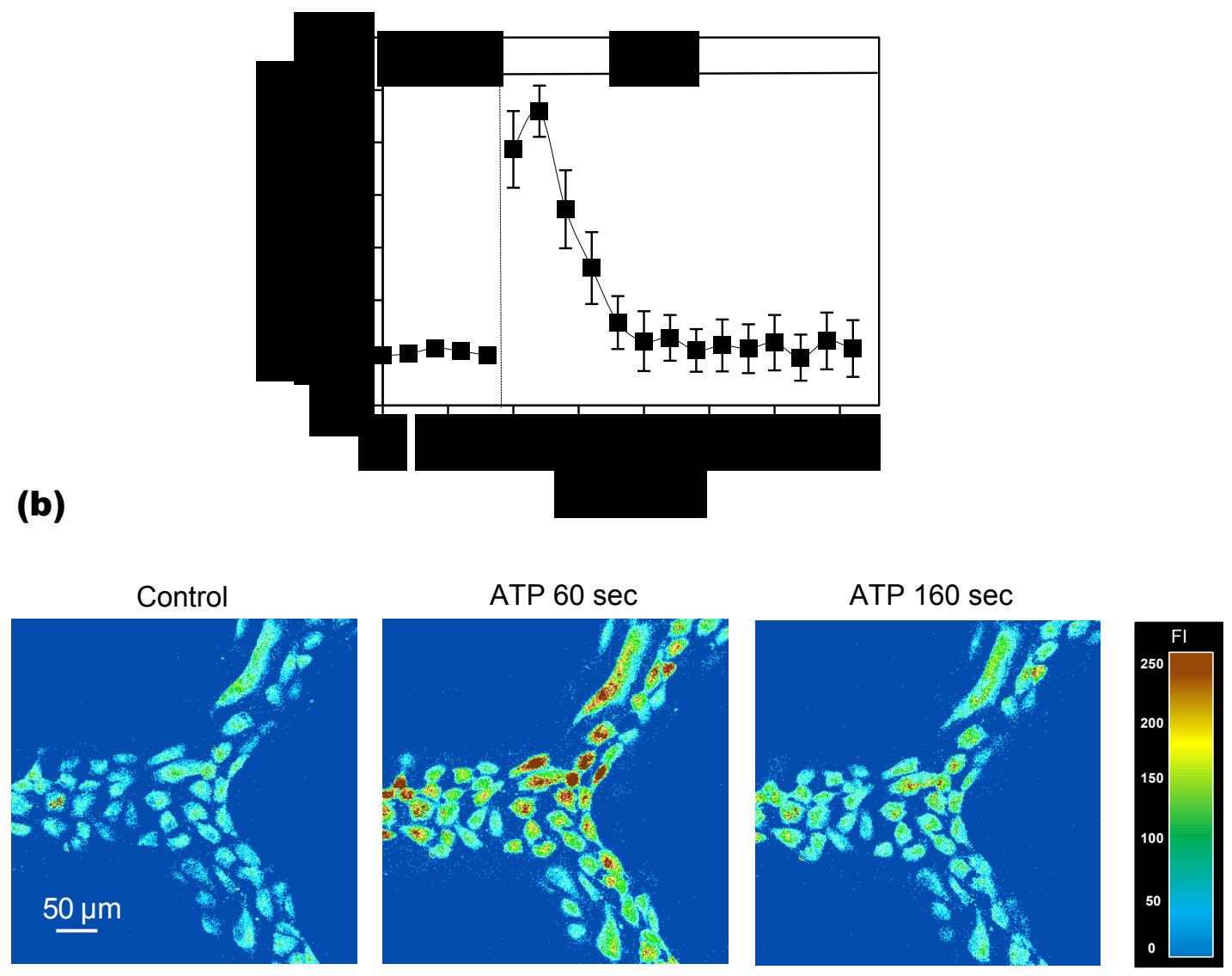

Figure 5 ATP-induced endothelial cell $\left[\mathrm{Ca}^{2+}\right]_{i}$ increase in HUVECs cultured microchannel network. (a) Time course of ATP-induced changes in endothelial $\left[\mathrm{Ca}^{2+}\right] \mathrm{i}$ from a representative experiment; (b) Fluo-4 confocal images from one representative experiment before and after the start of ATP perfusion at 60 and 160 seconds. 
Fig. 6

(a)

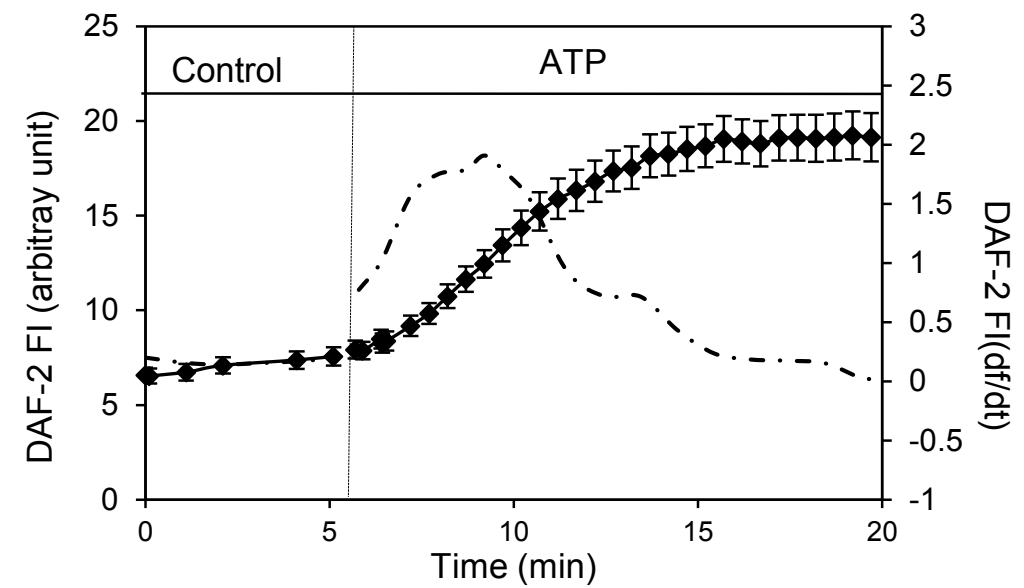

(b)

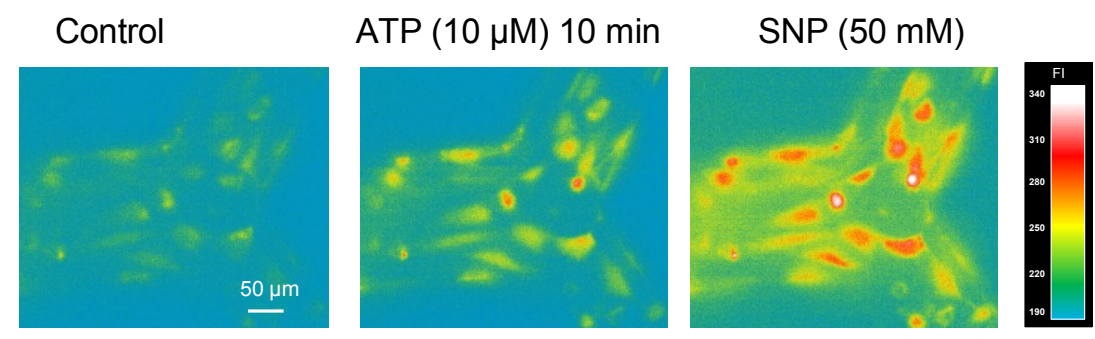

Figure 6 ATP-induced NO production increases in HUVECs cultured microchannel network. (a) Time dependent changes in FIDAF (left $Y$ axis) from a representative experiment before and after ATP $(10 \mu \mathrm{M})$ stimulation with continuous perfusion of DAF-2 DA at $5 \mu \mathrm{M}$. The 1st differential conversion of cumulative FlDAF (df /dt, right $Y$ axis) represents the NO production rate (dash-dotted line); (b) Representative DAF-2 fluorescence images. ATP induced significant increases in nitric oxide (NO)-DAF-2 fluorescence intensity $(\mathrm{FI})$ relative to control (left). FI was further increased with the application of the NO donor sodium nitroprusside (SNP). 
CHAPTER 6

GENERAL DISCUSSION, CONCLUSION AND FUTURE STUDIES 


\section{GENERAL DISCUSSION AND CONCLUSION}

Unchecked increase in microvessel permeability can lead to a variety of cardiovascular diseases. A deep and integrative understanding of the mechanisms of NO and shear stress participating in the regulation of microvascular permeability could provide new perspective into those diseases, which may eventually contribute to inventing potential therapeutic strategies.

The role of NO in regulating microvessel permeability has been debated for more than a decade. The study in Chapter 2 demonstrated for the first time that exogenously applied AP-CAV, a specific eNOS inhibitor, has been shown to not only inhibit inflammatory stimulus-induced increases in NO production and microvessel permeability, ${ }^{1-3}$ but also reduce basal NO production in endothelial cells lining microvessel walls. Furthermore, the study suggested that reducing basal NO by AP-CAV did not directly change basal microvessel permeability, but it increased the adhesive capacity of endothelial cells and promotes leukocyte adhesion via Src-mediated phosphorylation of ICAM-1. Meanwhile, a NO donor, SNP, abolished the effect of AP-CAV on increased ICAM-1 binding to a blocking antibody and leukocyte adhesion, and further supported that the anti-adhesive property of basal NO in maintaining the homeostasis of vascular walls.

In addition to the mechanism of CAV-induced leukocyte adhesion, we found that CAVinduced leukocytes adhesion does not increase microvessel permeability, which was further approved by the observation of the intact and continuous VE-cadherin on endothelial junction. Previously, our study showed that TNF-a-induced leukocyte adhesion and migration are not sufficient to cause increases in microvessel permeability. ${ }^{4}$ Although the interaction of leukocytes with endothelial cells has been considered as the critical event 
leading to permeability increase, there are also increasing evidences demonstrating the dissociation between leukocyte adhesion and changes in permeability. ${ }^{5,}{ }^{6}$ Studies in rat tracheal mucosa found that $94 \%$ of the gap junctions were distinct from sites of leukocyte adhesion or migration in the leaky venules. 7,8 Valeski et al demonstrated that blocking histamine-associated adherent leukocytes had no significant effect on leakage formation. ${ }^{9}$ Furthermore, previous studies from our lab and others suggested that it is the additional stimuli that induced leukocyte oxidative burst which, in turn, are responsible for the increased microvessel permeability. ${ }^{4,10-13}$ Our previous study demonstrated that fMLPinduced ROS production in adhered leukocytes to vascular wall was one of the mechanisms responsible for the increased permeability in TNF- $\alpha$ primed rat mesenteric venules. ${ }^{10}$ In further support of this hypothesis, in the current study, we observed a transient increase in microvessel permeability only upon fMLP $(10 \mu \mathrm{M})$ perfusion in the presence of AP-CAV-induced adherent leukocytes. Our finding that AP-CAV-induced leukocytes adhesion does not increase permeability consolidates the theory that adhesive interactions between leucocytes and endothelial cells is independent from the potential endothelial damage caused by leukocyte ROS release and therefore is dissociated from increases in microvessel permeability.

Other than chemical stimulation, mechanical force stimulation also has been suggested play an important role in the regulation of microvessel permeability. Wall SS is the tangential force exerted per unit area of the blood-endothelium surface. Post-capillary venules are the main sites where the permeability increase and recruitment of leukocytes occur during inflammation. Only a few in vivo studies investigating whether the shear stress (by either increase perfusion pressure or shear rate) affect microvessel $L p$ in frog or rat 
mesentery venules. ${ }^{14-16}$ Unfortunately, these studies provide contradicting results. In those studies, none of them involving blood cell components, which are always present in the blood stream. Our results in Chapter 3 indicated that in intact venules ATP released from RBCs in response to a sudden change of SS is responsible for the increases in $E C\left[\mathrm{Ca}^{2+}\right]_{i}$ and vascular permeability, whereas SS-induced EC NO production can be independent of increased EC $\left[\mathrm{Ca}^{2+}\right]$. It may provide the basic mechanisms that help to explain and predict the roles of acute changes in SS in different vasculature under both physiological and pathological conditions. Under physiological condition, variations in vascular geometry could cause the RBCs experienced high SS at small vessel region, which may release increased amount of ATP and cause increases in EC $\left[\mathrm{Ca}^{2+}\right]_{i}$ and gap formation at downstream vessels. Under pathological conditions, such as vascular lumen narrowing due to the atherosclerotic plaque formation, or injury-induced local cell proliferation, sudden increases in SS and increased ATP release may dramatically affect the downstream vessel segment permeability and exaggerate the local inflammation.

Other than the question we discussed in Chapter 3: 'How blood flow-generated shear stress regulates microvessel permeability?', another mystery waiting for resolving is 'how endothelial cells sense the shear stress?'. Up to now, there are several mechanosensor candidates have been proposed, such as ion channels, ${ }^{17}$ G-protein-coupled receptors (GPCRs), ${ }^{18}$ caveolae, ${ }^{19}$ adhesion proteins, ${ }^{20}$ and the glycocalyx. ${ }^{21}$ The glycocalyx is a layer of proteoglycans covering the endothelium. It has been indicated to be a mechanotransduction sensor for changes of shear stress. ${ }^{22}$ An impaired glycocalyx has been suggested to contribute to the increased leukocyte adhesion, hyperpermeability, and other microvascular dysfunctions associated with diabetes and other cardiovascular 
diseases. In Chapter 4, we found the impaired glycocalyx increases the endothelial susceptibility to shear stress, which augmented NO production in microvessels of diabetic rats. Similar enhanced NO production has also been found in pronase treated normal vessel. The results implicates that glycocalyx may serves to reduce the fluid shear stress at the surface of the membrane where most receptors are located that could serve as mechanosensor.

Besides in vivo study of microvessel, in Chapter 5 , we demonstrated in vitro formation of a microvessel network that recapitulates key features of microvessels in vivo and validate its utility for biological applications. Microfluidic model has been indicated could have a great potential for biological applications and bridging the gaps between in vitro and in vivo microvascular research. Comparing to other conventional in vitro models, our model is more physiological relevant. Firstly, endothelialized microchannels are under flow control, which mimics microscaled dimensions of in vivo microvessels with continuous flow. Secondly, immunofluorescence staining of VE-cadherin and F-actin demonstrated the formation of vascular endothelial junctions and well developed cytoskeleton, two important characters indicating well-maintained endothelial barrier functions. Thirdly, engineered microvessels EC transient $\left[\mathrm{Ca}^{2+}\right]$ i responses and increased NO production in response to agonist application are similar to those observed in individually perfused intact microvessels. Overall, it suggested that the developed microvessel model has the potential to bridge the gap between over-simplified in vitro tests and more expensive and laborintensive in vivo animal models for the microvascular signaling and functional studies. Also, by culturing an individual human subject's cells or perfusing human subject's blood or fluid, it offers valuable clinical significance under both physiological and pathological conditions. 
Overall, this dissertation demonstrated the important role of basal NO in the regulation of microvessel permeability and leukocyte adhesion, the critical role of blood flow generated shear stress in endothelial cell signaling and microvessel permeability in vivo, the effect of impaired glycocalyx in diabetic rat venules in its response to shear stress, and also the potential usage of microfluidic devices in microvascular research. 


\section{SIGNIFICANCE OF THE STUDY}

Despite years of studies, the exact roles of $\mathrm{NO}$ in the regulation of microvessel permeability remain controversial. The study in Chapter 2 was conducted in intact microvessels under basal conditions. Combined with our previous findings on inflammatory stimuli-induced NO and microvessel permeability, ${ }^{1-3,23}$ we revealed dual actions of NO on endothelial functions using the same experimental preparation, but under different conditions, which provides a unified perspective of the roles of NO in the regulation of endothelial function and microvessel permeability.

Previous studies of the effects of SS on vascular endothelium were mainly conducted in the absence of blood cell components either in cultured endothelial monolayers ${ }^{24,25}$ or in individually perfused microvessels. ${ }^{14-16}$ Whereas the studies of the effects of shear on blood cells, such as RBCs, usually do not involve vascular endothelium. ${ }^{26-28}$ Our studies by direct comparisons of intact microvascular responses to SS generated by uniform fluids, such as plasma or solutions containing Dextran 70, with SS generated by blood flow demonstrated that under physiological conditions, i.e. in blood perfused microvessels, the changes of blood flow affect endothelium not only by fluid-generated wall SS, but also by shear-induced ATP release from RBCs. Our results in Chapter 3 indicated that an increase of fluid-generated wall SS induces increased endothelial cell NO production, which does not involve an increase in endothelial $\left[\mathrm{Ca}^{2+}\right]_{i .}$ However, under in vivo conditions, i.e. in blood perfused vessels, the increased shear exerted on RBCs induces shear-magnitude dependent ATP release. This SS-dependent ATP release from RBCs causes increases in endothelial $\left[\mathrm{Ca}^{2+}\right]_{i}$ and endothelial gap formation, an indication of increases in microvessel permeability. By linking the effects of wall SS on endothelium with the effects of shear 
induced ATP release from RBCs on endothelium, our study provided new insight about how changes of blood flow affect vascular functions in vivo.

Most of the experiments in our study have been conducted in individually perfused microvessels with undisturbed surrounding circulation, which we consider extremely important. In order to translate the remarkable knowledge gained from in vitro studies into clinical therapeutic applications, it is essential to evaluate cellular and molecular mechanisms in their native state. Multidimensional protein identification revealed that $41 \%$ of proteins expressed in vivo are not detected in vitro, indicating that distinct in vivo protein expression is apparently regulated by the tissue microenvironment and cannot yet be duplicated in standard cell culture. ${ }^{29}$ This is specifically important for glycocalyx studies, because the glycocalyx under static cultured cell growth is not as well developed as that under physiological conditions. ${ }^{30}$ 


\section{FUTURE STUDIES}

The dissertation has demonstrated that NO and SS play important roles in the regulation of microvessel permeability. There are still many questions worth further exploration.

In Chapter 2, the timing of rapid increased ICAM-1 staining induced by AP-CAV perfusion ruled out the possibility of new ICAM-1 protein synthesis. However, our results could not enable us to rule out the possibility of membrane translocation of ICAM-1 in response to AP-CAV-induced NO reduction. TNF- $\alpha$-induced early phase leukocyte adhesion involves the activation of constitutive ICAM-1 on cell surface, which is demonstrated by protein analysis from cytoplasmic and membrane fractions in cultured cells. ${ }^{31}$ In order to pursue the detailed molecular mechanism of AP-CAV induced rapid increase of EC ICAM-1 affinity to leukocyte, with collaboration with an in vitro research group, we are going to first verify our finding on cultured cells and then using western blotting, Real time PCR and other molecular techniques to study the change of EC ICAM1 from both cytosol and cell membrane upon the stimulation of AP-CAV.

In Chapter 3, we found both plasma and blood-generated shear stress induced shearmagnitude dependent EC NO production increase. Interestingly, plasma perfusion generated change of shear stress caused more EC production than that of blood perfusion. However, eNOS $\operatorname{ser}^{1177}$ and $\mathrm{Thr}^{495}$ immunofluorescence staining revealed that blood perfusion resulted in same extent of eNOS activation as plasma did. We hypothesized that it may due to hemoglobin in the RBCs scavenge the EC NO induced by the blood perfusion. To test this hypothesis, we could use $\mathrm{CO}$ to uncover role of NO binding to hemoglobin. We could perfuse carbon monoxide blood into our microvessel to see whether it generated more EC NO production than that of blood perfusion. 
Also, in Chapter 3, we used carbenoxolone to test our hypothesis that Pannexin 1 channel is the one responsible for the ATP releasing from RBCs triggered by shear stress. Carbenoxolone is an unspecific gap junction blocker, and has been demonstrated strongly inhibited Pannexin $1\left((\right.$ IC50 $=5 \mu \mathrm{m}){ }^{32}$ Other than ubiquitously expressed Pannexin 1 channel, Pannexin family also has Pannexin 2, and Pannexin 3 channel as its family members. Pannexin 1 was first demonstrated to act as an ATP-permeant channel on RBCs in 2006. ${ }^{33}$ In order to rule out the Pannexin 2 and Pannexin 3's possibilities involving in shear stress induced ATP releasing from RBCs, we would further investigate the role of Pannexin 1 channel using Pannexin 1 channel genetic knockout mice.

The glycocalyx has been indicated as a mechanotransduction sensor to the fluid shear stress in many in vitro studies. ${ }^{22,34-36}$ Using enzyme degradation of its specific components impaired the shear stress induced NO production and increased permeability in vitro. ${ }^{37}$ In Chapter 4, our results suggested that loss or impaired EC glycocalyx enhanced EC NO production to shear stress. Based on the cell culture studies, removing hyaluronic acid $(\mathrm{HA})$, sialic acids (SA), heparin sulfate (HS) but not chondroitin sulfate (CS) abolished NO production. ${ }^{38}$ Some studies reported that removing HA, HS, CS blocked shear-induced increases in Lp. Our future study will use glycocalyx component-specific enzymes individually or in combination to degrade the glycocalyx, which enable us to differentiate the roles of different glycocalyx components in shear stress-induced NO production in intact microvessels. Meanwhile, since NO is an important regulator in microvessel permeability and leukocyte adhesion. It is also interesting to investigate the role of glycocalyx in microvessel permeability and leukocyte adhesion. 


\section{REFERENCE}

1. Zhou X, He P. Endothelial [Ca2+]i and caveolin-1 antagonistically regulate eNOS activity and microvessel permeability in rat venules. Cardiovasc Res. 2010;87(2):340-347.

2. Zhou X, He P. Temporal and spatial correlation of platelet-activating factor-induced increases in endothelial $[\mathrm{Ca}(2)(+)]$ i, nitric oxide, and gap formation in intact venules. Am J Physiol Heart Circ Physiol. 2011;301(5):H1788-1797.

3. Zhu L, Schwegler-Berry D, Castranova V, He P. Internalization of caveolin-1 scaffolding domain facilitated by Antennapedia homeodomain attenuates PAFinduced increase in microvessel permeability. Am J Physiol Heart Circ Physiol. 2004;286(1):H195-201.

4. Zeng $\mathrm{M}$, Zhang $\mathrm{H}$, Lowell $\mathrm{C}$, He $\mathrm{P}$. Tumor necrosis factor-alpha-induced leukocyte adhesion and microvessel permeability. Am J Physiol Heart Circ Physiol. 2002;283(6):H2420-2430.

5. He P. Leucocyte/endothelium interactions and microvessel permeability: coupled or uncoupled? Cardiovasc Res.87(2):281-290.

6. Harris NR, Benoit JN, Granger DN. Capillary filtration during acute inflammation: role of adherent neutrophils. Am J Physiol. 1993;265(5 Pt 2):H1623-1628.

7. McDonald DM. Endothelial gaps and permeability of venules in rat tracheas exposed to inflammatory stimuli. Am J Physiol. 1994;266(1 Pt 1):L61-83.

8. Baluk P, Bolton P, Hirata A, Thurston G, McDonald DM. Endothelial gaps and adherent leukocytes in allergen-induced early-and late-phase plasma leakage in rat airways. Am J Pathol. 1998;152(6):1463-1476.

9. Valeski JE, Baldwin AL. Effect of early transient adherent leukocytes on venular permeability and endothelial actin cytoskeleton. Am J Physiol. 1999;277(2 Pt 2):H569-575.

10. Zhu L, He P. fMLP-stimulated release of reactive oxygen species from adherent leukocytes increases microvessel permeability. Am J Physiol Heart Circ Physiol. 2006;290(1):H365-372.

11. Zhu L, Castranova V, He P. fMLP-stimulated neutrophils increase endothelial [Ca2+]i and microvessel permeability in the absence of adhesion: role of reactive oxygen species. Am J Physiol Heart Circ Physiol. 2005;288(3):H1331-1338.

12. Tinsley JH, Ustinova EE, Xu W, Yuan SY. Src-dependent, neutrophil-mediated vascular hyperpermeability and beta-catenin modification. Am J Physiol Cell Physiol. 2002;283(6):C1745-1751.

13. Jakus Z, Berton G, Ligeti E, Lowell CA, Mocsai A. Responses of neutrophils to antiintegrin antibodies depends on costimulation through low affinity Fc gamma Rs: full 
activation requires both integrin and nonintegrin signals. $J$ Immunol. 2004;173(3):2068-2077.

14. Neal CR, Bates DO. Measurement of hydraulic conductivity of single perfused Rana mesenteric microvessels between periods of controlled shear stress. J Physiol. 2002;543(Pt 3):947-957.

15. Williams DA. A shear stress component to the modulation of capillary hydraulic conductivity (Lp). Microcirculation. 1996;3(2):229-232.

16. Adamson RH, Sarai RK, Altangerel A, Clark JF, Weinbaum S, Curry FE. Microvascular permeability to water is independent of shear stress, but dependent on flow direction. Am J Physiol Heart Circ Physiol. 2013;304(8):H1077-1084.

17. Olesen SP, Clapham DE, Davies PF. Haemodynamic shear stress activates a K+ current in vascular endothelial cells. Nature. 1988;331(6152):168-170.

18. Gudi SR, Clark CB, Frangos JA. Fluid flow rapidly activates $G$ proteins in human endothelial cells. Involvement of $G$ proteins in mechanochemical signal transduction. Circ Res. 1996;79(4):834-839.

19. Rizzo V, Morton C, DePaola N, Schnitzer JE, Davies PF. Recruitment of endothelial caveolae into mechanotransduction pathways by flow conditioning in vitro. $\mathrm{Am} \mathrm{J}$ Physiol Heart Circ Physiol. 2003;285(4):H1720-1729.

20. Osawa M, Masuda M, Kusano K, Fujiwara K. Evidence for a role of platelet endothelial cell adhesion molecule-1 in endothelial cell mechanosignal transduction: is it a mechanoresponsive molecule? J Cell Biol. 2002;158(4):773-785.

21. Tarbell JM, Pahakis MY. Mechanotransduction and the glycocalyx. $J$ Intern Med. 2006;259(4):339-350.

22. Weinbaum $S$, Zhang $X$, Han $Y$, Vink $H$, Cowin SC. Mechanotransduction and flow across the endothelial glycocalyx. Proc Natl Acad Sci U S A. 2003;100(13):79887995.

23. Zhu L, He P. Platelet-activating factor increases endothelial $[\mathrm{Ca} 2+] \mathrm{i}$ and $\mathrm{NO}$ production in individually perfused intact microvessels. Am J Physiol Heart Circ Physiol. 2005;288(6):H2869-2877.

24. Sill HW, Chang YS, Artman JR, Frangos JA, Hollis TM, Tarbell JM. Shear stress increases hydraulic conductivity of cultured endothelial monolayers. Am J Physiol. 1995;268(2 Pt 2):H535-543.

25. Pang Z, Antonetti DA, Tarbell JM. Shear stress regulates HUVEC hydraulic conductivity by occludin phosphorylation. Ann Biomed Eng. 2005;33(11):15361545.

26. Edwards J, Sprung R, Sprague R, Spence D. Chemiluminescence detection of ATP release from red blood cells upon passage through microbore tubing. Analyst. 2001;126(8):1257-1260. 
27. Forsyth AM, Wan J, Owrutsky PD, Abkarian M, Stone HA. Multiscale approach to link red blood cell dynamics, shear viscosity, and ATP release. Proc Natl Acad Sci U S A.108(27):10986-10991.

28. Wan J, Ristenpart WD, Stone HA. Dynamics of shear-induced ATP release from red blood cells. Proc Natl Acad Sci U S A. 2008;105(43):16432-16437.

29. Durr E, Yu J, Krasinska KM, Carver LA, Yates JR, Testa JE, Oh P, Schnitzer JE. Direct proteomic mapping of the lung microvascular endothelial cell surface in vivo and in cell culture. Nat Biotechnol. 2004;22(8):985-992.

30. Barakat Al. Dragging along: the glycocalyx and vascular endothelial cell mechanotransduction. Circ Res. 2008;102(7):747-748.

31. Javaid K, Rahman A, Anwar KN, Frey RS, Minshall RD, Malik AB. Tumor necrosis factor-alpha induces early-onset endothelial adhesivity by protein kinase Czetadependent activation of intercellular adhesion molecule-1. Circ Res. 2003;92(10):1089-1097.

32. Bruzzone R, Barbe MT, Jakob NJ, Monyer H. Pharmacological properties of homomeric and heteromeric pannexin hemichannels expressed in Xenopus oocytes. J Neurochem. 2005;92(5):1033-1043.

33. Locovei S, Bao L, Dahl G. Pannexin 1 in erythrocytes: function without a gap. Proc Natl Acad Sci U S A. 2006;103(20):7655-7659.

34. Mulivor AW, Lipowsky HH. Role of glycocalyx in leukocyte-endothelial cell adhesion. Am J Physiol Heart Circ Physiol. 2002;283(4):H1282-1291.

35. Reitsma S, Slaaf DW, Vink H, van Zandvoort MA, oude Egbrink MG. The endothelial glycocalyx: composition, functions, and visualization. Pflugers Arch. 2007;454(3):345-359.

36. Weinbaum S, Tarbell JM, Damiano ER. The structure and function of the endothelial glycocalyx layer. Annu Rev Biomed Eng. 2007;9:121-167.

37. Mochizuki S, Vink H, Hiramatsu O, Kajita T, Shigeto F, Spaan JA, Kajiya F. Role of hyaluronic acid glycosaminoglycans in shear-induced endothelium-derived nitric oxide release. Am J Physiol Heart Circ Physiol. 2003;285(2):H722-726.

38. Pahakis MY, Kosky JR, Dull RO, Tarbell JM. The role of endothelial glycocalyx components in mechanotransduction of fluid shear stress. Biochem Biophys Res Commun. 2007;355(1):228-233. 


\title{
Sulei Xu
}

\section{CURRICULUM VITAE}

\author{
Research Assistant \\ West Virginia University, School of Medicine, Department of Physiology and Pharmacology \\ One Medical Center Drive, HSN-3068, WV 26506 \\ Cell 304-906-6514 \\ Email: sxu1@mix.wvu.edu
}

\section{EDUCATION}

- PhD in Cellular and integrative physiology

1/2009 to 10/2014 (expected), West Virginia University, Morgantown, WV

- B.S. in Bioengineering

9/2004 to $7 / 2008$, Shanghai University, Shanghai, China

\section{PROFESSIONAL EXPERIENCE}

\section{- Research Assistant}

West Virginia University, Department of Physiology and Pharmacology, School of Medicine, 1/2009 to date, supervised by Dr. Ping He

Project 1: Differentiate the roles of endogenous endothelial S1P subtype receptors in the regulation of permeability in intact microvessels

Project 2: Investigate the roles of exogenously applied caveolin-1 peptide in endothelial basal nitric oxide production, ICAM-1 expression, leukocyte adhesion, and adhesion-induced changes in microvessel permeability

Project 3: Investigate cellular and molecular mechanisms of how shear stress regulates microvessel functions under physiological and pathological conditions

Project 4: Investigate the enhanced permeability responses to inflammation in streptozotocin-induced diabetic rat venules: Rho-mediated alterations of actin cytoskeleton and VE-cadherin

Project 5: Investigate endothelial nitric oxide and calcium response to ATP in microfluidic device

\section{- Research Assistant}

Shanghai University, School of Life Science, 8/2007 to 12/2008, supervised by Dr. Fuxue Chen

Project 1: Investigate the structure, Function and Modulation of KATP Channels (in responsible for aortic ring organ bath vascular function assay)

Project 2: Down-regulation of $67 L R$ reduces the migratory activity of Human Glioma Cell ( involving in cell culture, plasmid siRNA transfection and wound-healing assay)

\section{PUBLICATIONS}

- Xu, S, Yokota, S, He, P. Shear stress induced ATP release from erythrocytes increases 
endothelial calcium and vascular permeability in intact rat venules. (In preparation)

- Xu, S, Yuan, D and He, P. The impaired glycocalyx increases the endothelial susceptibility to shear stress resulting in augmented nitric oxide production in microvessels of diabetic rats. (In preparation)

- $\mathrm{Li}, \mathrm{X}^{*}, \mathbf{X u}, \mathbf{S}^{*}, \mathrm{He}, \mathrm{P}$, Liu, Y. In Vitro Recapitulation of Functional Microvessels for the Study of Endothelial Shear Response, Nitric Oxide and $\left[\mathrm{Ca}^{2+}\right]_{i}$. (Submitted, *Shared first authorship)

- Yuan, D, Xu, S and He, P. Enhanced permeability responses to inflammation in streptozotocininduced diabetic rat venules: Rho-mediated alterations of actin cytoskeleton and VE-cadherin. Am J Physiol Heart Circ Physiol. 2014, 307(1):H44-H53.

- Xu, S, Zhou, X, Yuan, D, Xu, Y and He, P. Caveolin-1 scaffolding domain promotes leukocyte adhesion by reduced basal endothelial nitric oxide-mediated ICAM-1 phosphorylation in rat mesenteric venules. Am J Physiol Heart Circ Physiol. 2013, 305(10):H1484-93.

- Zhang, G, Xu, S. Qian, Y and He, P. Sphingosine 1-phosphate (S1P) prevents permeability increases via activation of endothelial S1P receptor 1 in rat venules. Am J Physiol Heart Circ Physiol, 2010, 299(5): H1494-1504

- Chen, F, Ren, W, Yang, Y, Shen, D, Zong, Y, Xu, S, Di, S, Qian Y and Ji, Y. Reciprocal effects of conditioned medium on cultured glioma cells and neural stem cells. Journal of Clinical Neuroscience. 2009, (12):1619-1623

- Duan, Y, Ren, W, QIAN ,Y, Zheng, D, Xu, Y, Zong, Y, Xu, S and Chen, F. Down-regulation of 67LR Reduces the Migratory Activity of Human Glioma Cell, Acta Agriculturae BorealiOccidentalis Sinica, 2008, (04):52-57

\section{ABSTRACTS}

- $\mathbf{X u}, \mathbf{S}$ and He, P. Changing shear stress-induced ATP release from erythrocytes increases endothelial calcium and vascular permeability independent from nitric oxide production in intact rat venules. FASEB, 2014

- Petrides, P, Tan, Z, Xu, S, Guo, G, Huber, J, Rosen, C and He, P. Ischemic stroke-induced increases in circulating microparticles promote leukocyte adhesion in intact microvessels. FASEB, 2014.

- $\mathbf{X u}, \mathbf{S}$ and He, P. The impaired glycocalyx increases the endothelial susceptibility to shear stress resulting in augmented nitric oxide production in microvessels of diabetic rats. FASEB, 2013

- Xu, S, Yuan, D and He, P. Shear stress-induced differential endothelial signaling and changes in microvessel permeability in the presence and absence of erythrocytes in intact rat venules. Vascular Biology, 2013.

- Yuan, D, Wang, M, Xu, S and He, P. Time course of changes in microvessel permeability in streptozotocin (STZ)-induced diabetic rats. FASEB, 2013.

- Xu, S, Yuan, D, He, P. Shear stress generated by different fluid compositions induces differential endothelial signaling in intact venules. FASEB, 2012.

- Xu, S, Zhou, X, Yuan, D, Xu, Y and He, P. Caveolin-1 scaffolding domain induces leukocyte 
adhesion without increasing permeability in intact rat mesenteric venules. FASEB, 2011.

- Yuan, D, Zhou, X, Xu, S, He, P. Streptozotocin-Induced Diabetes Enhances Microvessel Permeability Responses to Acutely Applied Inflammatory Mediator in Rat Mesenteric Venules. FASEB, 2011.

\section{AWARDS AND FELLOWSHIP}

- 2014 Award for selected Oral presentation, Van Liere Convocation, School of Medicine, West Virginia University, Morgantown, USA

- 2014 E.J. Van Liere Memorial Convocation and Research Day Poster Presentation (First Place award), West Virginia University, Health Science Center

- 2013 Society for Experimental Biology and Medicine Young Investigator Award

- 2012 American Heart Association, Great River Affiliate Winter Predoctoral Fellowship (07/01/2012-06/30/2014)

- 2011 Benjamin Zweifach Graduate Student Travel Award in EB Poster section

- 2010 E.J. Van Liere Memorial Convocation and Research Day Poster Presentation (First Place award), West Virginia University, Health Science Center

- 2006-2007, First-Prize academic scholarship(merit based), Shanghai University, China

- 2005-2006, First-Prize academic scholarship(merit based), Shanghai University, China

- 2004-2005, Second-Prize academic scholarship(merit based),Shanghai University, China

\section{MEMBERSHIPS OF PROFESSIONAL ORGANIZATIONS}

- 2013-present Student member of Society for Experimental Biology and Medicine

- 2012-present Student member of American Heart Association

- 2011-present Student member of American Physiology Society

- 2011-present Student member of Microcirculatory Society 